\title{
Spaceborne Synthetic-Aperture Imaging Radars: Applications, Techniques, and Technology
}

\author{
CHARLES ELACHI, MEMBER IEEE, T. BICKNELL, ROLANDO L. JORDAN, AND CHIALIN WU
}

\begin{abstract}
In the last four years, the first two Earth-orbiting, spaceborne, synthetic-aperture imaging radars (SAR) were successfully developed and operated. This was a major achievement in the development of spaceborne radar sensors and ground processors. The data acquired with these sensors extended the capability of Earth resources and oceansurface observation into a new region of the electromagnetic spectrum. This paper is a review of the different aspects of spaceborne imaging radars. It includes a review of: 1) the unique characteristics of spaceborne SAR systems; 2 ) the state of the art in spaceborne SAR hardware and SAR optical and digital processors; 3) the different data-handling techniques; and 4) the different applications of spaceborne SAR data.
\end{abstract}

\section{INTRODUCTION}

II N JUNE 1978, the Seasat satellite was put into orbit around the Earth with a synthetic-aperture imaging radar (SAR) as one of the payload sensors [12]. The Seasat SAR provided, for the first time, synoptic radar images of the Earth's surface with a resolution of $25 \mathrm{~m}$. The success of this complex sensor was a major technological advance, and it opened up a new dimension in our capability to observe, monitor, and study the Earth's surface [31], [39]. In November 1981, the second imaging radar was successfully operated from space on the Shuttle [33]. The Shuttle Imaging Radar-A (SIR-A) acquired images over a variety of regions around the world with an imaging geometry different from the one used by the Seasat SAR.

Spaceborne photography became available in the early 1960's with the advent of the space age. This was followed in the late 1960 's and 1970's with the acquisition of multispectral visible and infrared (IR) imagery, thermal imagery, and passive microwave imagery. These sensors allowed us to acquire information about the surface by studying its emitted energy in the microwave and IR regions of the spectrum and the reflected energy in the visible and near-IR regions. All these sensors are passive in nature, i.e., they detect the energy which is generated by the sun or the surface.

The SAR imaging sensor provides information about the surface by measuring and mapping the reflected energy in the microwave region, thus extending the capability of sensing the surface properties into a new dimension. In addition, because it uses its own energy and operates at a relatively long wavelength, it acquires surface imagery at all times, i.e., day or night and through cloud cover. Thus it has the unique capability required for continuous monitoring of dynamic surface phenomena.

The imaging resolution of passive sensors is equal to their angular resolution (i.e., observing wavelength over aperture size) multiplied by the range between the sensor and the area

Manuscript received February 1, 1982; revised July 29, 1982. The research described in this paper was performed under Contract with the National Aeronautics and Space Administration. The submission of this paper was encouraged after the review of an advance proposal.

The authors are with the Jet Propulsion Laboratory, California Institute of Technology, Pasadena, CA 91109. or object being imaged. Thus the size of the resolution element increases linearly with the observing wavelength and sensor altitude, and is inversely proportional to the aperture size. In the optical and IR regions, very high resolution is achievable from orbit with reasonable size apertures because of the short operating wavelength. In the microwave region, the operating wavelength is relatively large, and apertures of many hundreds of meters to many kilometers are required to achieve high resolution of tens of meters or less. This, of course, is impractical at the present time.

The SAR sensor circumvents this limitation by using the ranging and Doppler tracking capability of coherent radars to acquire high-resolution images of the surface from orbital altitudes. Two neighboring targets are separated by their differential time delay and Doppler history, neither of which is a function of the distance to the sensor. Thus the resolution of a SAR system is independent of the sensor altitude. This unique advantage does impose some restrictions on the sensor imaging swath, antenna size, and power requirements. The basic properties of spaceborne SAR systems are discussed in Section II and the technological aspects of the sensor are presented in Section III.

Because the SAR uses the Doppler history to achieve high resolution in one of the spatial dimensions, each pixel is generated by processing a large number of successive echoes. This leads to a large number of arithmetic operations in order to generate the image. This arithmetic complexity is beyond the present capability of real-time processors. The development of digital processors for spaceborne SAR data is a very active and challenging research field. This aspect of the SAR system is the subject of Section IV.

A very elegant way of executing a large number of the types of calculations required for SAR data processing can be achieved with optical techniques. In actuality, the first airborne SAR systems used optical processors. In Section V, we discuss how these types of processors have been adapted to handle the unique aspects of spaceborne SAR data.

Once the image has been formed, a number of post-imageformation processing steps are used to maximize the usefulness of the information in the radar imagery. These include, among others, radar image registration to multispectral visible/IR images, automatic textural analysis, and speckle statistical analysis. These techniques are addressed in Section VI.

Section VII addresses interpretation techniques and applications of spaceborne SAR data. We review the techniques and image features that an interpreter uses in extracting information about the surface, and we present a variety of specific examples in the different areas of the Earth Sciences-geology, oceanography, glaciology, and agriculture.

At the end of each section, we briefly discuss the developments expected in the near future. In Section VIII, we present 


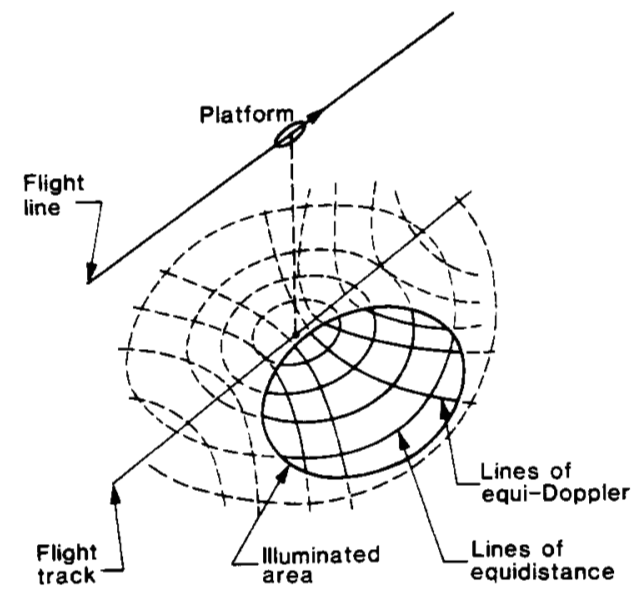

Fig. 1. Coordinate system for SAR image formation. A set of circles and hyperbolas define the equirange and equi-Doppler lines, respectively.

our opinion of the major challenges in the field of SAR remote sensing for the 1980's and briefly review the development being planned for spaceborne SAR systems during this decade.

\section{SPACEborne SAR Principle}

In the synthetic-aperture technique, the Doppler information in the returned echo is used simultaneously with the timedelay information to generate a high-resolution image of the surface being illuminated by radar [16], [70], [26], [44], [52]. The radar usually "looks" to one side of the moving platform-to eliminate right-left ambiguities-and perpendicular to its line of motion. It transmits a short pulse of coherent electromagnetic energy toward the surface. Points equidistant from the radar are located on successive concentric spheres. The intersection of these spheres with a flat surface gives a series of concentric circles centered at the nadir point (see Fig. 1). The backscatter echoes from objects along a certain circle will have a well-defined time delay but different Doppler characteristics.

Points distributed on coaxial cones, with the flight line as the axis and the radar as the apex, provide identical Doppler shifts of the returned echo but different delays. The intersection of these cones with a flat surface gives a family of hyperbolas (Fig. 1). Objects on a specific hyperbola will provide equi-Doppler returns. Thus if the time delay and Doppler information in the returned echoes are processed simultaneously, the surface can be divided into a coordinate system of concentric circles and coaxial hyperbolas (Fig. 1), and each point on the surface can be uniquely identified by a specific time delay and specific Doppler. The brightness that is assigned to a specific pixel (picture-resolution element) in the radar image is proportional to the echo energy contained in the time-delay bin and the Doppler bin, which corresponds to the equivalent point on the surface being imaged. The resolution capability of the imaging system is thus dependent on the measurement accuracy of the differential time delay and differential Doppler (or phase) between two neighboring points on the surface.

In actuality, the situation is somewhat more complicated. The radar transmits a pulsed signal in order to obtain the timedelay information. To obtain the Doppler information unambiguously, the echoes from many successive pulses are required with a pulse-repetition frequency (PRF) which meets the Nyquist sampling criterion. Thus as the moving platform passes

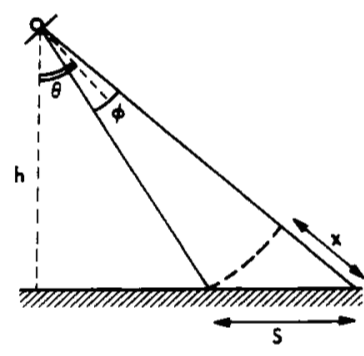

(a)

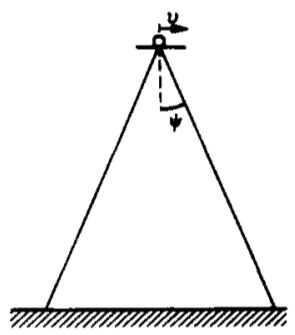

(b)
Fig. 2. Imaging radar viewing geometry: (a) in the range plane, (b) in the azimuth plane.

over a certain region, the received echoes contain a complete Doppler history and range-change history for each point on the surface that is being illuminated. These complete histories are then processed to identify uniquely each point on the surface and to generate the image. This is why a very large number of operations are required to generate each pixel in the image. Such is not the case with optical sensors. A simplified comparison is that the radar sensor generates the equivalent of a hologram of the surface, and further processing is required to obtain the image. This processing can be done either optically or digitally.

One unique feature of the synthetic-aperture imaging radar is that its resolution capability is independent of the platform altitude. This is a result of the fact that the image is formed by using the Doppler history and the differential time delays, none of which is a function of the range from the radar to the surface. This unique capability allows the acquisition of highresolution images from satellite altitude as long as the received echo has sufficient strength above the noise level.

In this section, we will discuss the main features of spaceborne SAR systems. These include: azimuth and range ambiguities, range walk, the effects of Earth's rotation and orbital characteristics, surface interactions, SAR image characteristics, key tradeoff parameters influencing the sensor design, and nonconventional SAR systems.

\section{A. Ambiguities}

There will be ambiguity in the response if the PRF is so high that return signals from two successive transmitted pulses arrive simultaneously at the receiver. This is called range ambiguity. Conversely, if the PRF is so low that the return is not sampled at the Nyquist rate, there will be Doppler azimuth ambiguity.

The upper limit of the PRF is fixed by the range or elevation beamwidth of the SAR antenna. A view of the beam geometry in the range plane is shown in Fig. 2. In order to avoid the situation in which the far edge echo arrives at the receiver simultaneously with the near edge echo from the following pulse, it is necessary that

$$
T>2 X / c
$$

i.e.,

$$
T>\frac{2 h}{c} \frac{\tan \theta}{\cos \theta} \phi=\frac{2 \lambda h}{c W} \sin \theta / \cos ^{2} \theta
$$

or

$$
\text { PRF }<c W \cos ^{2} \theta / 2 \lambda h \sin \theta
$$

where $T$ is the time between successive pulses, $\lambda$ is the radar wavelength, $W$ is the antenna width, $\theta$ is the look angle, $h$ is the platform height, $c$ is the speed of light, $X$ is the slant range 
swath, and $\phi$ is the beamwidth (see Fig. 2(a)). The above equations assume that $\phi$ is small and the pulse length is much smaller than $T$. The Earth's surface curvature is neglected.

The lower limit of the PRF is imposed by the requirement that the PRF must equal or exceed the maximum Doppler shift of the return signals. Targets at the center of the broadside beam will return signals with zero Doppler shift. Targets ahead of broadside center are characterized by a positive Doppler and those behind by negative Doppler frequencies. If the return of a target is shifted in frequency by an amount equal to the PRF, the receiver will be unable to distinguish the pulsed return signal from that of a target on broadside center. Thus the PRF has to be sufficiently high to exceed the maximum Doppler shift of targets located at beam edge. The Doppler shift $f_{D}$ due to a radial velocity $v_{r}$ is given by

$$
f_{D}=\frac{2 v_{r}}{c} f
$$

where $f$ is the radar frequency. By referring to Fig. 2(b), $v_{r}=v$ $\sin \psi \simeq v \psi$. The angle $\psi$ to the first null of the one-way radar illumination beam is $\Psi=\lambda / L$ where $L$ is the antenna length. Thus the lower limit of the PRF is

$$
\mathrm{PRF}_{\text {low }}=f_{D}=\frac{2 v}{L}
$$

The two limitations derived above give

$$
\frac{c W \cos ^{2} \theta}{2 \lambda h \sin \theta}>\mathrm{PRF}>2 v / L
$$

which can be rewritten as

$$
W L>\frac{4 v \lambda h \sin \theta}{c \cos ^{2} \theta} .
$$

This condition imposes a minimum size on the antenna area $(W L)$ which is required to avoid ambiguities. Equation (7) should be used only in preliminary calculations. In actual design, the Earth curvature should be taken into account.

\section{B. Point-Target Response}

The simplest way to understand the SAR image-formation mechanism and the necessary processing is to consider the case of a bright point target on the surface. Let us consider the case of a linearly phase-modulated waveform $W(t)$.

$$
W(t)=A(t) e^{i\left[\omega_{0} t+(a / 2) t^{2}\right]}
$$

where

$$
\begin{array}{ll}
A(t)=1, & \text { for } n T-\tau<t<n T+\tau \\
A(t)=0, & \text { otherwise }
\end{array}
$$

where $T$ is the pulse repetition period, $2 \tau$ is the pulse length, $\omega_{0}$ is the carrier frequency, $a$ is the chirp rate, and $n$ is an integer. While the target is within the antenna beam, the returned echo $E(t)$ will be modulated by the antenna pattern (which is neglected here) and by a phase shift proportional to the round-trip delay; i.e.,

$$
E(t) \sim A(t) e^{i\left[\omega_{0} t+(a / 2) t^{2}+r(t)\right]}
$$

where

$$
r(t)=\sqrt{h^{2}+X^{2}+v^{2} t^{2}} .
$$

If the successive echoes are frequency shifted down to video and their instantaneous amplitude (not envelope) is used to modulate the light intensity of an optical film scanner, the

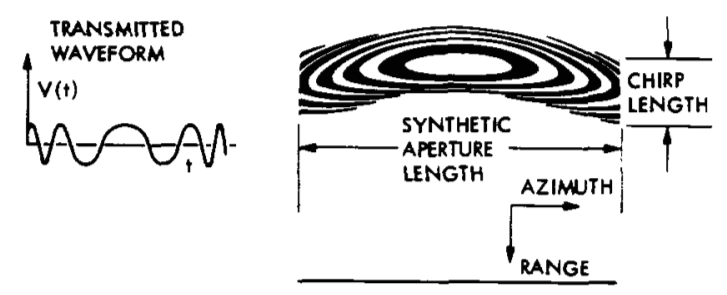

Fig. 3. Point-target response showing the range-curvature effect.

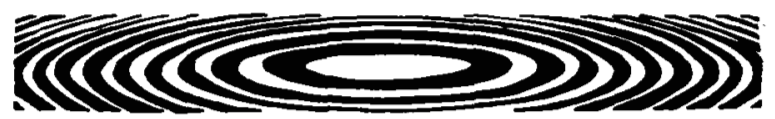

Fig. 4. Point-target response for an airborne system when the range curvature is negligible.

corresponding record will be what is shown in Fig. 3. Each vertical line corresponds to one echo which has a chirp behavior (see insert in Fig. 3). Successive echoes are slightly shifted in phase relative to each other because of the platform motion and the resulting Doppler shift. The whole pattern is also bent because of the large change in the distance from the spaceborne platform to the target while the target is in the beam. This bending in the pattern is called range curvature. In the case of aircraft systems, this bending is negligible (see Fig. 4).

In reality, the point-target response is even more complex. Because of the Earth's rotation, which adds an additional Doppler component, the orbit ellipticity, and errors in the spacecraft attitude, the point-response pattern is asymmetric (as shown in Fig. 5).

In its simplest form (Fig. 4), the synthetic-aperture point response can be easily compressed into the image point. This can be done optically or digitally. In the optical processing approach, the modulated signal film acts like a Fresnel lens and will focus incident laser light into a focal point which is the image. In the digital processing case, a two-dimensional correlation, or two successive one-dimensional (one along each axis) correlations with a reference function, will compress the signal data to form the image. In the real case (Fig. 5), the range walk and range curvatures have to be taken into account and corrected for. These can be estimated from knowledge of the spacecraft state vector. However, some feedback mechanism is usually required to achieve accurate compression. The compression techniques are discussed in detail in Section IV.

\section{Orbital and Environmental Factors}

The satellite orbital plane is fixed in space. Targets on the Earth's surface move with a linear velocity which is dependent on their latitudes. It is maximum at the equator and zero at the poles. The resulting Doppler effect leads to an asymmetry in the point response as discussed above. Three methods can be used to compensate for this effect [77]: i) rotating the antenna beam in yaw about the satellite nadir to include the zero Doppler-frequency direction. (Because the rotation angle varies with latitude, this requires the satellite attitude to be continuously adjusted.); ii) continuously adjusting the receiver local-oscillator frequency with respect to the transmitted frequency to cancel the effect of the Doppler from the Earth rotation; and iii) subtracting the Doppler due to Earth rotation from the received signal during processing. The last approach was used by the Seasat SAR.

In a similar way, orbit eccentricity causes an altitude change 


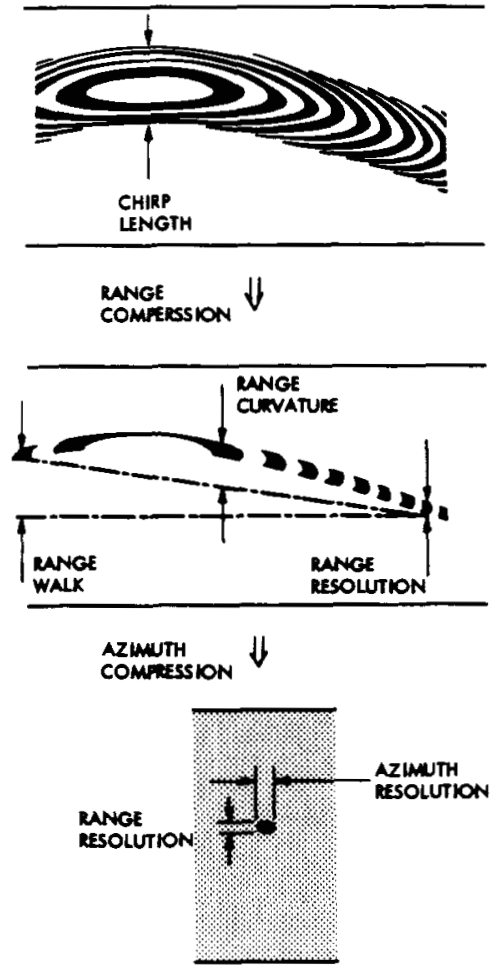

Fig. 5. Point-target response showing the spaceborne SAR case where range curvature and range walk are present. Also illustrated are the processing steps as well as other parameters discussed in the paper.

and displaces the image in the track direction. With descending altitude, the velocity vector will dip below the local horizon. The zero Doppler-frequency direction is perpendicular to the satellite-velocity vector so that the image will shift rearward relative to the subsatellite point $[44],[77]$. The image shift is given by

$$
s=\frac{h}{v} \frac{d h}{d t} \cos \theta .
$$

Similar to the Earth-rotation effect, compensation for altitude change can be accomplished by rotating the antenna boresight axis in yaw about the local satellite nadir, by trimming the receiver local-oscillator frequency, or during processing. It should be noted that since the orbit perigee will migrate, the orbital position of maximum altitude change rate will migrate.

The orbital effects (ellipticity and attitude errors) are deterministic and well behaved. Therefore, they can be estimated directly or by analyzing the data using clutterlock feedback. However, undeterministic effects could be encountered with spaceborne radar systems. Amplitude and phase random scintillations are caused by ionospheric irregularities [1], [21]. The phase scintillation would degrade the radar image if the granularity along a synthetic-aperture length leads to phase shifts in excess of a small fraction of a radian. The magnitude of the phase granularity can be calculated from time-delay formulations given by Burns and Friemouw [17], and it is expected to be of appreciable impact only for systems operating at frequencies lower than $1 \mathrm{GHz}$.

\section{Range Curvature and Range Walk}

During integration along the synthetic-aperture length, the target range does change in excess of the range resolution, as illustrated in Fig. 3. This effect is called range curvature [56]. If $R_{0}$ is the nearest range to the target and $R_{m}$ is the maximum range at the edge of the synthetic aperture, then

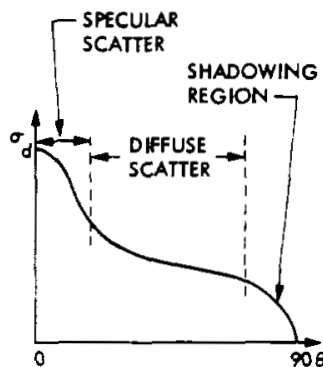

(a)

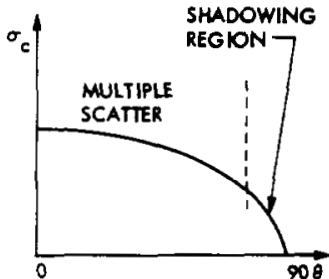

(b)
Fig. 6. Simplified sketch of the backscatter cross section behavior as a function of incidence angle and polarization: (a) direct backscatter, (b) depolarized backscatter. The two curves are not to the same scale.

$$
R_{m}=\sqrt{R_{0}^{2}+\left(\frac{v \zeta}{2}\right)^{2}}=R_{0}+\frac{1}{8} \frac{(v \zeta)^{2}}{R_{0}}
$$

where $\zeta=\lambda R_{0} / 2 v r_{z}=$ integration time, and $r_{z}$ is the azimuth resolution. The range curvature is given by

$$
R_{C}=R_{0}-R_{m}=\frac{1}{8} \frac{(u \zeta)^{2}}{R_{0}}=\frac{\lambda^{2} R_{0}}{32 r_{z}^{2}} .
$$

Relative to the range slant resolution $r_{r}$, then

$$
\frac{R_{c}}{r_{r}}=\frac{\lambda^{2} R_{0}}{32 r_{r} r_{z}^{2}} \text {. }
$$

For the Seasat SAR system, $r_{r}=r_{z}=7 \mathrm{~m}, \lambda=0.25 \mathrm{~m}$, and $R_{0}=850 \mathrm{~km}$. The range curvature is equal to

$$
\frac{R_{c}}{r_{r}}=4.3
$$

This implies that a point migrates through 4.3 resolution elements during the formation of the synthetic aperture. This effect must be taken into account during processing (see later).

The range walk is defined as the difference in the range to a point target when it leaves the illumination beam relative to when it enters the beam (see Fig. 5). The range walk is a function of attitude errors, orbit ellipticity, and linear velocity of the target. It can be one to two orders of magnitude larger than the range resolution.

Range curvature and range walk are called range migration.

\section{E. Surface Interaction Mechanisms}

The brightness of an individual pixel in a radar image is a direct representation of the backscatter cross section of the corresponding area on the surface being imaged. The backscatter cross section is mainly dependent on the physical (e.g., slope, roughness, and near-surface inhomogeneities) and electrical (e.g., dielectric constant) properties of the surface, as well as the radar wave characteristics-incidence angle, frequency, and polarization state. A number of interaction mechanisms contribute to the backscattered energy [47]. Bragg scattering is dominant at relatively large incidence angles and for relatively well-organized surfaces. Rayleigh scattering is dominant in the case of discrete objects. Specular reflection is of particular importance at near-vertical incidence, and multiple scattering plays a key role in depolarization of the incident wave.

In its most simplified form, the behavior of the backscatter cross section $\sigma$ as a function of incidence angle is shown in a sketch form in Fig. 6 . Because of the numerous surface parameters which influence the backscatter cross section, a complete description of the surface properties will most likely require multiple observations with different radar parameters. If the 
radar system operates at a single frequency, it can be seen from Fig. 6 that large incidence angles are more favorable for observing surface topography because of the resulting shadowing. Small incidence angles are also desirable because of the high sensitivity of $\sigma_{d}$ to variations in the surface slope. Intermediate incidence angles are more favorable for observing surface roughness. Multiple incidence-angle observation will allow the acquisition of information about different surface properties, and will allow the use of stereo techniques.

Multiple-frequency observation is of particular interest at intermediate angles in order to acquire a measure of the spectral characteristics of the surface roughness. Multiple-polarization observations provide information about volumetric and multiple scattering, and will tend to supress the effects of the large-scale topography (i.e., slope changes).

\section{F. Noise in SAR Systems: Thermal and Speckle Noise}

If we neglect the quantization noise, the two types of noise to be considered in a radar design are the thermal noise and the speckle noise.

The thermal noise is an additive noise which is due to the noise temperature at the input of the receiver. The signal-tothermal noise ratio can be improved by increasing the transmitted peak power or decreasing the receiver temperature. This is the type of noise encountered in real-aperture radar systems.

The speckle noise is due to the coherent nature of the SAR image-formation process. This noise gives the radar image a fine texture similar to what is observed when a scene is illuminated with laser light. The speckle noise is proportional to the reflected signal power. Thus it is a multiplicative noise which cannot be reduced by increasing the transmitted power. The speckle noise can only be reduced by averaging independent looks.

Before detection, the speckle noise is assumed to be a zeromean Gaussian noise on each of the in-phase and quadrature components. After detection, the intensity in an image pixel has then an exponential probability distribution of the form

$$
p(I)=\frac{1}{I_{0}} e^{-I / I_{0}}
$$

where $I_{0}$ is the average return in the absence of speckle. As multilooks are coherently added, the speckle variance is reduced and becomes chi-squared $\left(\chi^{2}\right)$ distributed with $2 N$ degrees of freedom where $N$ is the number of independent looks. This gives a probability density function for the intensity of an image pixel as

$$
p(I)=\frac{1}{\Gamma(N)} \frac{1}{I_{0}}\left(\frac{I}{I_{0}}\right)^{N-1} e^{-I / I_{0}} .
$$

This distribution has a mean of $\mu=N I_{0}$ and a standard deviation $\Sigma=\sqrt{N} I_{0}$.

One measure of an image radiometric resolution, for a stationary scene, is

$$
Q=10 \log \left(\frac{\mu+\Sigma}{\mu-\Sigma}\right)=10 \log \left(\frac{\sqrt{N}+1}{\sqrt{N}-1}\right)
$$

which clearly shows that higher values of $N$ are desirable. However, for $N \geqslant 25$ (e.g., $Q=1.8 \mathrm{~dB}$ ), large increases in $N$ give only small improvement in $Q$. This should be traded off with the fact that increasing $N$ immediately degrades the image resolution in a proportional way.

A detailed theoretical study with experimental verification of the speckle noise property was given by Bennett and McConnell [8].

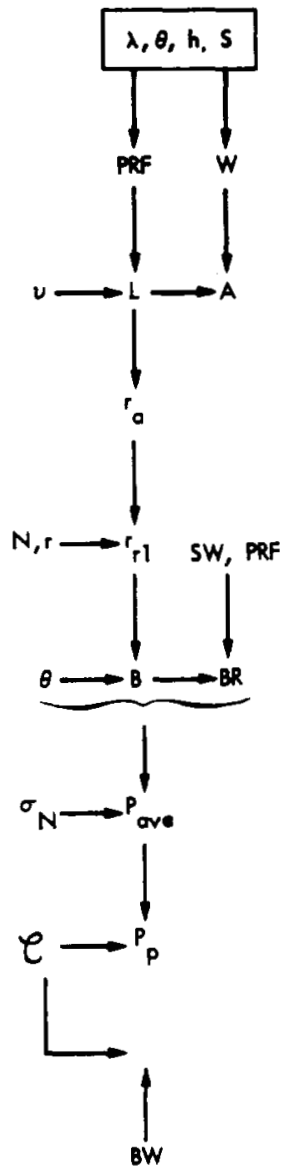

Fig. 7. Sketch showing the steps of one procedure to determine the different parameters for a spaceborne SAR system.

\section{G. Key Tradeoff Parameters}

In defining and optimizing the design of a radar system, a large number of parameters have to be determined and traded off. Sophisticated computer programs have been developed at the Jet Propulsion Laboratory (JPL), Pasadena, CA, in connection with the development of the Seasat SAR and SIR-A, and with the planning of Venus orbiting-imaging radar (VOIR) and future Shuttle and free-flying SAR sensors.

For illustrative purposes, a simplified step-by-step parameters estimation procedure is described here. Other procedures could be equally well used. The most involved steps are not included, such as integrated ambiguities or sidelobes level, image-calibration parameters, pixel-brightness statistics, Earth ellipticity, spacecraft-attitude effects, system nonlinearities, sample-digitization level, effect of data-link bit error rates, sampling noise, etc.

We assume that the radar wavelength $\lambda$, illumination incidence angle $\theta$, and image swath width $S$ are fixed by the scientific objective. We also assume that the orbit altitude $h$ and platform velocity $v$ are given, and we neglect the surface curvature. In a complete design procedure, these parameters $\lambda, \theta$, $S$, and $h$ are usually input variables which can also be varied to optimize the design.

The steps of the procedure are given in a schematic form in Fig. 7. The pulse-repetition frequency is derived from the requirement that the echoes from successive pulses should not overlap. Thus (see Fig. 2)

$$
\text { PRF } \leqslant C S \sin \theta / 2
$$




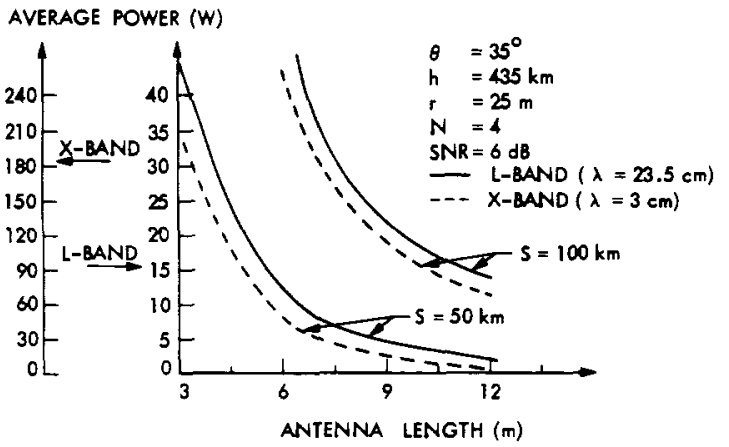

Fig. 8. Illustrative examples of tradeoff curves.

$$
W=\lambda h / S \cos ^{2} \theta .
$$

From the maximum PRF, we can then determine the minimum antenna length such that the Doppler shift is sufficiently sampled by the PRF.

$$
L>\frac{2 v}{\text { PRF }} .
$$

The antenna width and minimum length give the minimum antenna area $A=W L$. The one-look azimuth resolution is theoretically equal to half the antenna length for a fully focused system [16]. Thus

$$
r_{a l}=L / 2 \text {. }
$$

If we assume that the desired resolution $r$ (assumed to be the same in azimuth and range) and total number of looks $N$ are given, then the one-look range resolution is

$$
r_{r l}=r^{2} / N r_{a l}
$$

The bit rate is then determined by the bandwidth, the sampling duty cycle, and the number of bits per sample.

The next key parameter is the average transmitted power required to achieve a noise-equivalent backscatter $\sigma_{N}$. This is the surface backscatter cross section which will give a thermal signal-to-noise ratio (SNR) of unity (i.e., $0 \mathrm{~dB}$ ).

$$
P_{\text {ave }}=\frac{8 \pi k T \lambda B h^{3} \mathrm{PRF}}{\sigma_{N} c L W^{2} \cos ^{3} \theta}
$$

and the peak transmitted power is

$$
P_{p}=\frac{8 \pi k T \lambda B^{2} h^{3}}{\sigma_{N} c L e W^{2} \cos ^{3} \theta}
$$

where $e$ is the compression ratio. The dispersed pulse length is equal to

$$
\tau=e / B \text {. }
$$

The above equations give the simplified relationships between the radar parameters. In defining a SAR system, the designer can change a number of input parameters to optimize the system. Fig. 8 gives some illustrative examples of tradeoff curves.

\section{H. Nonconventional SAR Systems}

A number of coherent imaging radar systems have been proposed which use a slight variation relative to the conventional synthetic-aperture technique discussed above. Some of these systems were proposed in order to get around the limitation imposed on the swath width by the ambiguities; others have the capability of surface imaging at a constant incident angle (but variable azimuth angle). Some of these systems are dis-

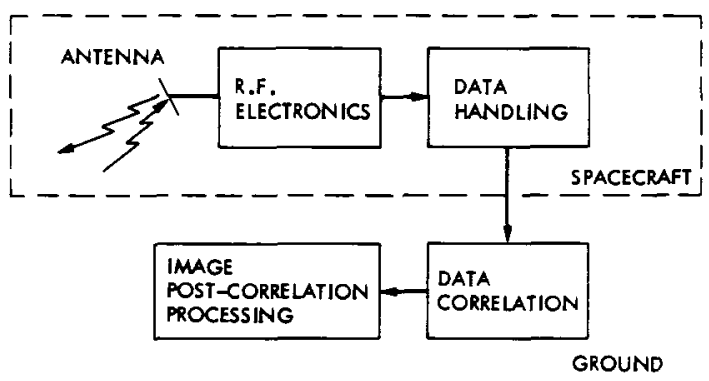

Fig. 9. Simplified block diagram of a typical SAR system showing its main elements.

cussed here briefly. These include the burst-mode SAR, the scan SAR, the geosynchronous SAR, and the bistatic SAR. All of these systems basically use the concept of range-Doppler processing to generate two-dimensional images.

In the conventional SAR, a continuous array along the flight is synthesized to achieve the high resolution. Repetitive subarrays can also be used to improve resolution relative to a realaperture system. These can be achieved by turning on the radar for a predetermined time period and then turning it off for another time period, and so on. This is called the burstmode SAR. With this approach, the power consumption is reduced by the turnoff duty cycle; however, there is a proportional loss in the resolution or number of looks.

One variety of the burst mode is to keep the radar turned on all the time, sequentially stepping the beam position, however, to illuminate neighboring swath track on the ground. The physical beam of the antenna initially illuminates one subswath long enough to synthesize a radar image covering the entire area of the beam. The beam is then pointed to the neighboring subswath for an equal length of time, and so on. The whole cycle is then repeated when the spacecraft moves about the length of the antenna footprint. This mode was proposed and studied by Moore, Classen, and Lin (see [62] and [76]). In effect, a burst mode is being used for each subswath. Cutrona [26] proposed the use of a wide-beam illuminator and simultaneous narrow receiving beams for the different subswaths. Classen and Eckerman [18] proposed a variant of this idea in which a constant incident angle, but variable illumination direction, could be achieved by illuminating the surface with a wide conical beam and by using narrow sub-beams for reception.

To synthesize a long aperture, the radar platform must be moving relative to the target. Thus a geostationary orbiting radar cannot use the synthetic-aperture technique. However, a geosynchronous radar in an inclined orbit will undergo a mutating motion which would allow aperture synthesis. The geosynchronous SAR was studied by Tomiyasu [76], [77]. The main advantage of such a SAR is that it could map a large limited area (such as the U.S. and Central America) repetitively every $24 \mathrm{~h}$. However, the power requirements and antenna size are relatively large though feasible.

In a bistatic SAR, the transmitter and receiver are not collocated. A synthetic aperture can be achieved with either the receiver, or transmitter, or both in motion. A number of configurations have been studied [76] and Elachi et al. [35].

\section{SPACEBORNE SAR SENSORS}

A SAR system can be divided into five major elements (Fig. 9). The antenna is the interface between the on-board RF electronics and free space. It radiates the electromagnetic energy toward the object to be sensed, and it collects the backscattered energy. The RF electronics consists of the transmitter subsystem which generates the RF signal and the receiver 


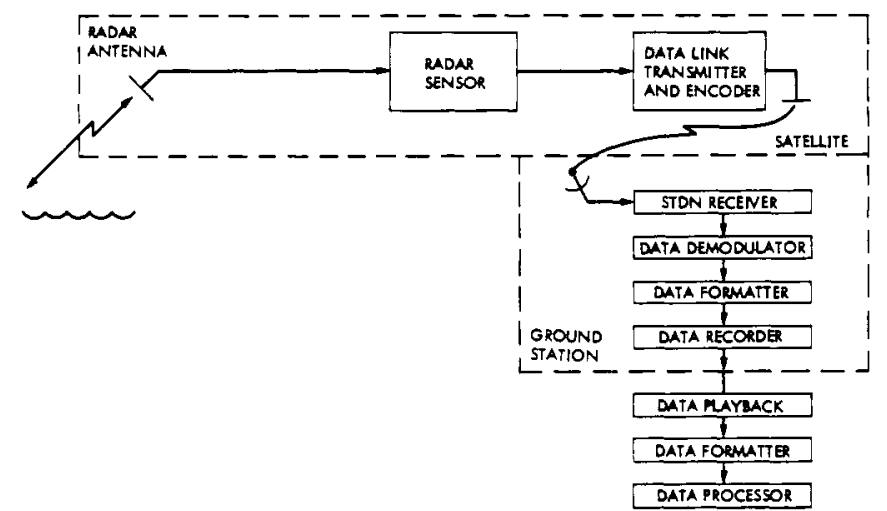

Fig. 10. Simplified block diagram of the Seasat SAR.

subsystem which detects the returned energy and converts it into a form which can be handled by the data-handling element. This element, in tum, reformats the data and transmits them to the data correlator. The output of the data-handling element could be either a digital tape with a digital record of the returned echoes or an optical film with an analog record of the returned echoes. This record contains what is commonly called the "signal data." These first three elements are referred to as the "SAR sensor." They are discussed in this section.

The data-correlator element transforms the "signal record" into an "image record" which is a two-dimensional representation of the surface reflectivity. This is the stage at which a recognizable image is acquired. These image data are then processed by an image post correlation processor for geometric and radiometric calibration, and for registration with other data sets acquired with similar (other radar data acquired at different frequency, polarization, or incidence angle) or different (visible or IR) imaging sensors. These last two elements are the subjects of Sections IV, V, and VI.

The first spaceborne SAR system was flown on the Apollo 17 spacecraft around the Moon. It was intended for subsurface sounding of the lunar crust $[67],[66]$. This sensor, called the Apollo Lunar Sounder Experiment (ALSE) radar was not specifically designed for surface imaging, and, therefore, will be only briefly discussed in this paper.

The first spaceborne SAR which was specifically designed to acquire synoptic high-resolution images of a planetary surface is the Seasat SAR which operated in orbit around the Earth during a 100-day period in the summer of 1978 [12]. The Seasat SAR [49], [48] incorporated some of the most advanced sensor technology of the late 1970's, and it is described here in detail. More recently, an imaging SAR was flown on the second Shuttle flight in November, 1981 [30]. This sensor [42], called the SIR-A, is very similar to the Seasat SAR as far as the electronics are concerned. Only its unique and additional features are discussed here.

\section{A. The Seasat SAR}

The Seasat SAR sensor system, shown in Fig. 10, consists of a planar array antenna, a transmitter/receiver RF sensor, an analog data link, a data formatter, and a high-density digital recorder subsystem which outputs the "signal record." The characteristics of the sensor are summarized in Table I. It was designed to provide continuous strips of radar imagery with a $100-\mathrm{km}$ swath at a resolution of $25 \mathrm{~m}$ from an orbital altitude of $800 \mathrm{~km}$. During its 100 days of operation, it acquired a voluminous set of images of the Earth's surface corresponding to a total imaged area of about 126 million square kilometers
TABLE I

SEAsat-A SAR System Characteristics

\begin{tabular}{ll}
\hline Satellite altitude & $800 \mathrm{~km}$ \\
Wavelength & $0.235 \mathrm{~m}$ \\
Theoretical resolution & $25 \times 25$ min on the surface at 4 looks
\end{tabular}

$\begin{array}{ll}\text { Swath width } & 100 \mathrm{~km} \\ \text { RF bandwidth } & 19 \mathrm{MHz} \\ \text { Iransmit pulse length } & 33.4 \mathrm{Hsec} \\ \text { Pulse repetition range } & 1463 \text { to } 1640 \mathrm{pps} \\ \text { Iransmit time bandwidth product } & 634 \\ \text { Radar transmitter peak power } & 1000 \mathrm{~W} \\ & \\ \text { Sensitivity ime control range } & 9 \mathrm{~dB} \\ \text { Data recorder bit rate } & 110 \mathrm{Mbits} / \mathrm{sec} \text { (5 bits per word) } \\ \text { Radar DC pover } & 500 \mathrm{~W} \\ \text { Radar antenna dimenstons } & 10.7 \mathrm{by} 2.16 \mathrm{~m} \\ \text { Radar antenna gatn } & 35 \mathrm{~dB}\end{array}$

(including multiple coverage). Because the radar data were directly linked to a limited number of specially equipped ground stations, the coverage was limited to the central and northern American hemisphere, western Europe, the North Atlantic, the North Pacific, and the northern polar regions.

Antenna: The Seasat-A antenna system consists of a 10.74by $2.16-\mathrm{m}$ phased-array system deployed after orbit insertion. This deployed antenna is configured to fly with the long dimension along the spacecraft-velocity vector and bore-sighted at an angle of $20.5^{\circ}$ from the nadir direction in elevation (cone) and $90^{\circ}$ from the nominal spacecraft-velocity vector (clock). The antenna dimensions $(10.74 \mathrm{~m} \times 2.16 \mathrm{~m})$ are dictated by a desire to limit range and Doppler ambiguities to acceptably low levels (see (6), (17)-(19)). At a nominal $20.5^{\circ}$ look angle from nadir, a total beamwidth in elevation of $6.2^{\circ}$ is required to illuminate a $100-\mathrm{km}$ swath on the Earth's surface from an $800-\mathrm{km}$-high orbit. Thus the antenna crosstrack dimension is $2.16 \mathrm{~m}$ to limit the radiation to these sets of angles (see (18)). The area illuminated on the surface of the Earth is from 240 to $340 \mathrm{~km}$ to the right of the subspacecraft point. The antenna elements in elevation are weighted to limit sidelobes in the cross-track direction. The minimum antenna along-track length is limited by a desire to keep azimuth sampling ambiguities to an acceptably low level (see (19)) while the maximum along-track length is determined by the requirement to illuminate a sufficiently large patch of terrain to allow processing of the data to achieve $25-\mathrm{m}$ resolution in azimuth with four looks.

These two requirements limit the antenna length along the velocity vector to between 10.5 and $14 \mathrm{~m}$. The $10.74-\mathrm{m}$ antenna length was dictated by the available volume within the satellite shroud. The level of integrated ambiguities in azimuth was estimated to be between -18 and $-24 \mathrm{~dB}$, depending on the selected PRF and processor bandwidth in azimuth.

The antenna subsystem consists of eight mechanically deployed, electrically coupled, flat microstrip panels. This array is shown in Fig. 11 in both stowed and deployed configurations. The construction of the microstrip is depicted in Fig. 12, and the deployment and extension mechanization is shown in Fig. 13.

$R F$ Sensor: The radar RF sensor provides the antenna with 


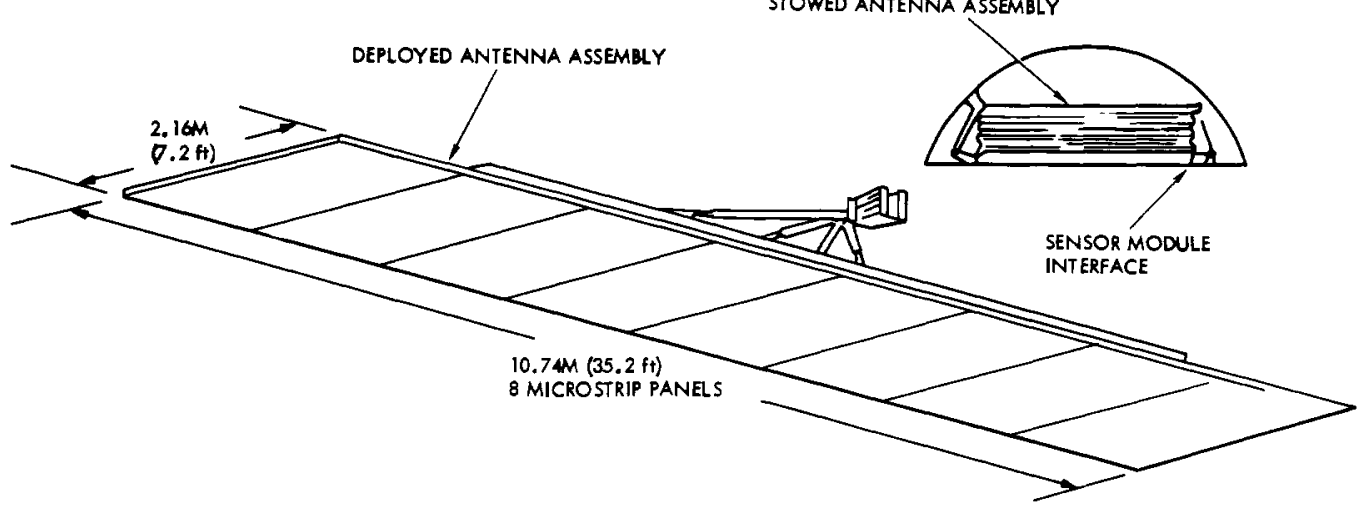

Fig. 11. Seasat SAR antenna shown in its stowed and deployed configurations.

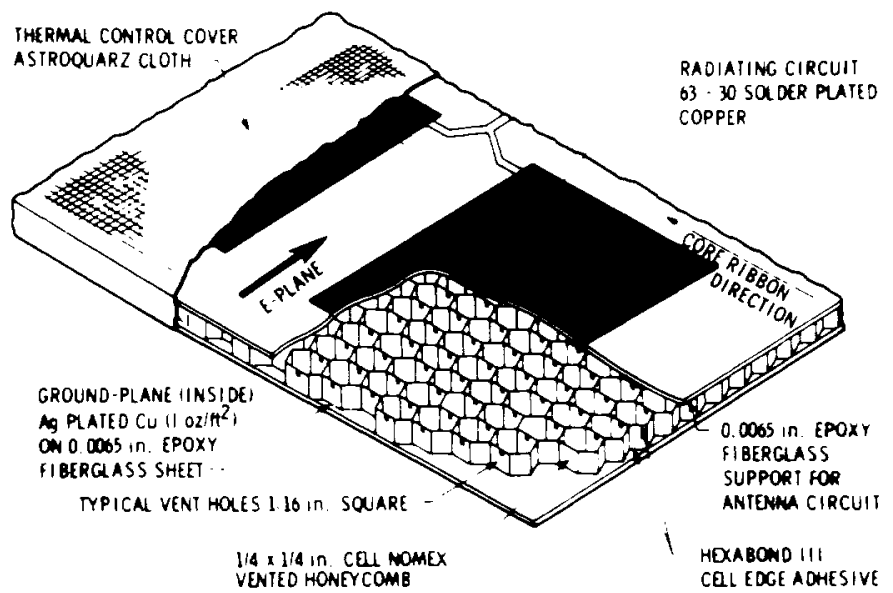

Fig. 12. Construction of the microstrip honeycomb pannels which were used in the Seasat and SIR-A antennas.

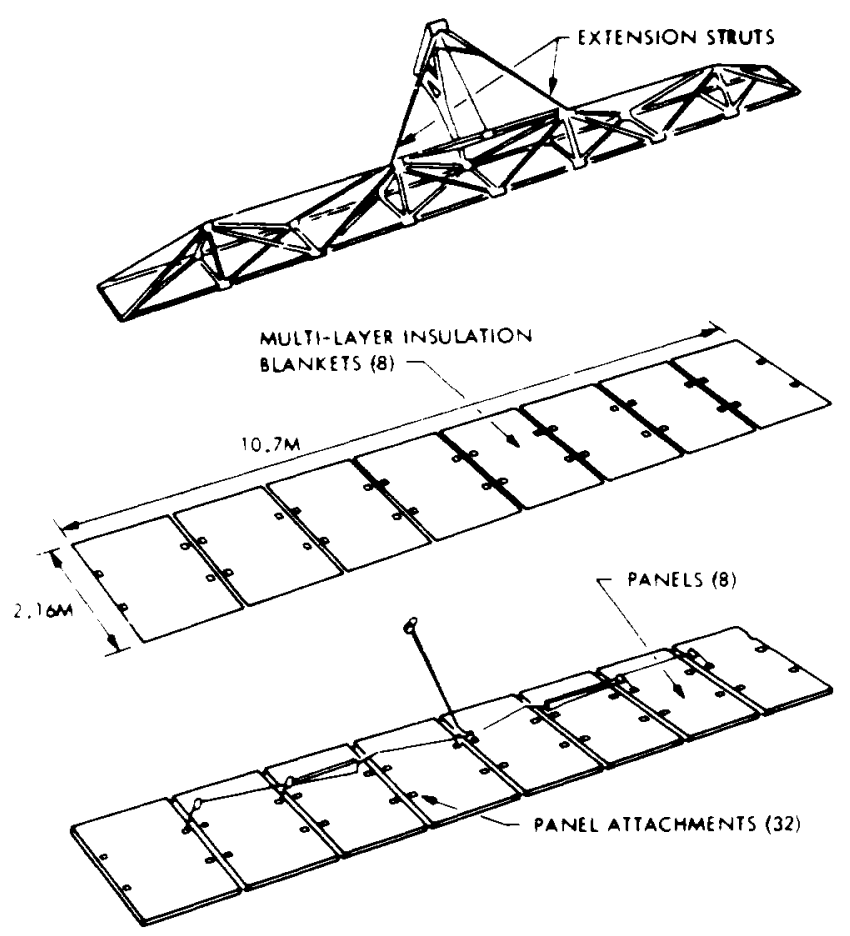

Fig. 13. Seasat SAR antenna mechanical components and assemblies. a series of high-power coherent pulses of energy at $L$-band and amplifies the weak return echoes collected by the antenna. The radar sensor consists of four subassemblies: transmitter, receiver, logic and control, and power converter. A diagram of the sensor is shown in Fig. 14, and the principal sensor parameters are tabulated in Table II.

To obtain an adequate SNR from a system whose range resolution is $25 \mathrm{~m}$ on the surface and which utilizes a solidstate transmitting device, it is necessary to use a long transmitted pulse and pulse-compression techniques to reduce the peak-power requirement. The output of the transmitter assembly is, as a result, a linearly swept frequency-modulated pulse (or chirp) having a 634:1 compression ratio. It is generated in a surface acoustic wave (SAW) device located in the chirp generator subassembly of the transmitter assembly. The output of the transmitter is coupled to the antenna subsystem through an output combiner. A portion of the output (leakage) is also impressed on the receiver input where a load is placed in the circuit each time the transmitter operates. This prevents the leakage pulse from burning out the input stage of the receiver.

Echo returns are coupled into the receiver assembly through the output network in the transmitter. Because the echo's intensity is expected to vary in proportion to the variation of antenna gain with angle, a sensitivity time control (STC) has been incorporated in the receiver. The STC action, initiated by satellite stored commands, linearly decreases the receiver gain by $9 \mathrm{~dB}$ during the first half of the return-echo period, and then increases the gain until the end of the echo has been received. The application of the STC results in a nearly uniform signal (echo) return for a uniform scattering field, and, as a result, the dynamic range required to transmit the resultant data to Earth is reduced by $9 \mathrm{~dB}$.

The sensor-receiver output is sent to the data link along with timing and frequency references derived from the SAR system local oscillator (STALO). The STALO generates a very stable (in frequency) signal at a nominal frequency $f_{s}\left(f_{s}=91.059\right.$ $\mathrm{MHz}$ ), a portion of which is delivered to the multiplier assembly. Another portion of this signal is used to derive both squarewave clock and sine-wave signals at $f_{s} / 3$, which are used in synchronizing other sensor electronics subsystem functions. The frequency multiplier assembly provides signals at $3 f_{s}, 9 f_{s}$, and $18 f_{s}$. The $3 f_{s}$ and $18 f_{s}$ signals are delivered to the chirp generator where, along with a portion of the STALO signal, $f_{s}$, they are used to generate the linear FM pulse (chirp) signal at the carrier frequency of $14 f_{s}$. The signal at $9 f_{s}$ and a por- 
RDAN: SEASAT-A SAR SYSTEM

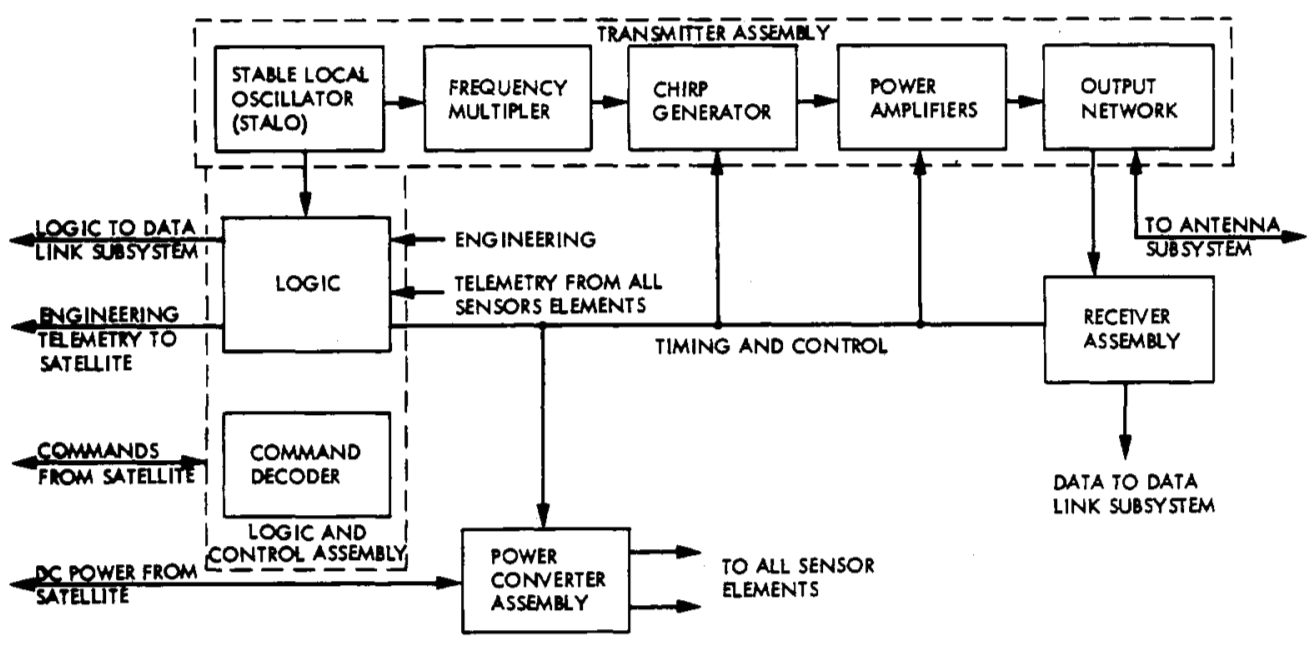

Fig. 14. Block diagram of the Seasat SAR sensor.

TABLE II

SENSOR EleCtronics Subsystem IMPLEMENTATION

\begin{tabular}{|c|c|}
\hline Electronics & Implementa:lon \\
\hline Center frequency & $1274.8 \pm 0.31 \mathrm{maz}$ \\
\hline Bandwidth & $19.05 \pm .05 \mathrm{MHz}$ \\
\hline STALO frequency & $91.059 \pm .0022 \mathrm{Mfiz}$ \\
\hline STALO stabillty & 3 parts $/ 20^{10}$ in $5=111$ iseconds \\
\hline Pulse width & $33.9 \pm 0.8 \mathrm{isec}$ \\
\hline Peak power & 1000 W nominal \\
\hline Puise envelope rise tire & $90 \mathrm{nsec}$ \\
\hline Pulse envelope fall cige & $90 \mathrm{nsec}$ \\
\hline Pulse repetition frequencies & $1464,1540,1580,1647 \pm .1 \mathrm{PPS}$ \\
\hline No1se temperature & 650 Kelvin, nominal \\
\hline Recelver gain control steps & 8 \\
\hline Gain control step spactsg & $3 \pm 0.3 \mathrm{~dB}$ \\
\hline Gain control range & 77 to $98 \mathrm{~dB}$ \\
\hline STC gatn variation & 9 \\
\hline Receiver gain flatness & $\pm 0.33 \mathrm{~dB}$ \\
\hline Receiver gain stability $\left(0-60^{\circ} \mathrm{C}\right)$ & $\pm 1.0 \mathrm{~dB}$ \\
\hline Receiver bandwidth ( $3 \mathrm{~dB}$ ) & $22 \pm 0.2 \mathrm{viz}$ \\
\hline Receiver phase ripple & $4.0^{\circ}$ dev. from quadratic \\
\hline Transmitter FM slope & $0.5622 \mathrm{xiz} / \mathrm{isec}$ \\
\hline Iransmitter phase response & $3^{\circ} \mathrm{rms}$ \\
\hline
\end{tabular}

tion of the signal at $f_{s}$ are delivered directly to the data-link subsystem along with a signal derived from the PRF event which divides the interpulse interval into 4096 sectors.

The remaining two assemblies in the sensor electronics subsystem are the logic and control as well as the power converter. They provide the primary electrical interface with the satellite. The logic and control assembly receives commands from the satellite, decodes them, and causes the sensor electronics subsystem to assume one of a number of operating modes. In addition, the logic and control interfaces between the satellite and the intra-subsystem engineering telemetry. The powerconverter assembly provides the stable, isolated power required by all the SAR subsystems.

Data-Link Subsystem: The purpose of the data-link subsystem is to transmit the radar echo to the ground for digitization, storage, and subsequent processing. The link also maintains the phase and time references necessary to the processing function, thus providing a unity transfer function. The chosen implementation technique was a linear $S$-band modulator/ transmitter/receiver/demodulator combination. This choice was governed chiefly by the available frequency spectrum/ bandwidth.

In addition to the basic requirement for linearity to preserve the SAR coherent information, the inclusion of necessary phase and timing signals placed an additional burden on the linear property of the link. The data-link subsystem performed the following functions:

a) Translated the $L$-band echo return to $S$-band.

b) Orthogonally combined the offset video with both PRF and STALO reference.

c) Amplified the result linearly (with negligible phase error) for transmission.

On the ground, the data-link subsystem:

a) Translated the composite $S$-band signal to an offset video frequency centered at $11.25 \mathrm{MHz}$.

b) Coherently demodulated the signal with the spacecraft local oscillator.

c) Removed the link-induced Doppler from the composite signal,

d) Reconstructed the PRF and STALO signals from the video.

Ground Station Subsystem: The Seasat SAR system required unique equipment at the ground receiving station. Only three U.S. stations (Fairbanks, Goldstone, and Merritt Island), one Canadian station (Shoe Cove), and one British station (Oak Hangar) were equipped to receive the SAR data. The SAR operations were limited to only the time periods when the satellite was in view of one of those stations.

The ground station subsystem consists of a data formatter and a high-density digital recorder. Upon the receipt of a trigger signal from the data-link subsystem demodulator as- 


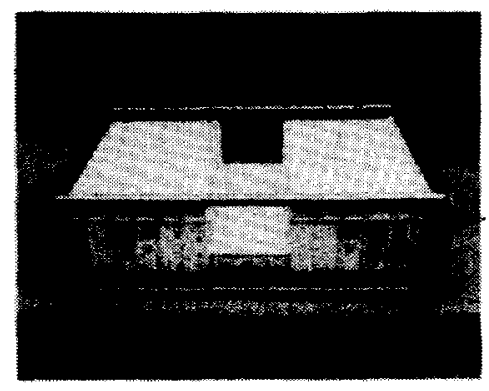

ELECTRONICS

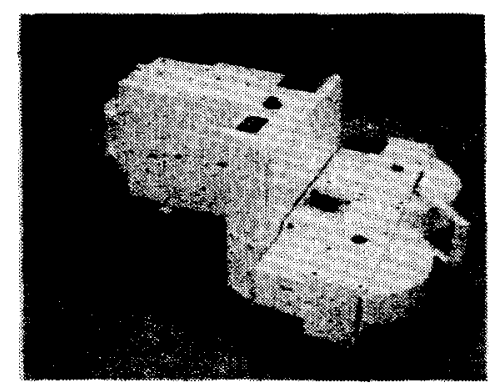

OPTKAL RECORDER

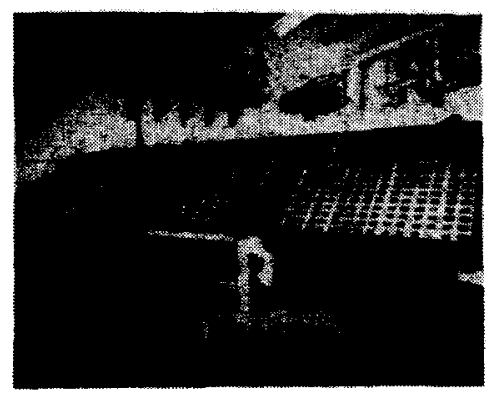

ANTENNA
Weight: $128 \mathrm{~kg}$

Size: $120 \times 85 \times 26 \mathrm{~cm}$

Peak Power Transmitted: 1100 watts

Average Power : 650 watts

Frequency: $1278 \mathrm{MHz}$

Brequency: $6 \mathrm{MHz}$

Pulse Length: 30.4 $\mathrm{sec}$ (chirped)

Pulse Length: $30.4 \mu \mathrm{sec}$ (chirped)
PRF: variable between $1464 \mathrm{~Hz}$ and $1824 \mathrm{~Hz}$
Weight: $70 \mathrm{~kg}$

Size (envelope): $108 \times 65 \times 43 \mathrm{~cm}$

Average Power: 135 watts

Film Capacity: 1100 meters

Film Speed: $36 \mathrm{~mm} / \mathrm{sec}$

Film Speed: $36 \mathrm{~mm} / \mathrm{sec}$

Fig. 15. Photographs of the SIR-A sensor and antenna.

sembly, the data formatter accepts and digitizes the analog offset video signal furnished by the demodulator. Digitization occurs only during the period $(\sim 300 \mu \mathrm{s})$ when the SAR video return is expected. The resulting 13680 samples, which are generated at a rate of $227 \mathrm{Mbits} / \mathrm{s}$, are temporarily stored within the data formatter. Information on the operational status of the data formatter and the demodulator is also collected and retained.

The video samples, the status information, and the GMT time are formatted and sent to the high-density digital recorder (HDDR) at a rate of about $113 \mathrm{Mbits} / \mathrm{s}$. The HDDR records this high-rate stream on 1 -in width magnetic tape. Recording uses 40 (of 42) parallel tracks on the tape at a recording speed of $150 \mathrm{in} / \mathrm{s}$. Parity is included on each track and timing information is carried on one of the remaining tracks.

The output "signal" tape is then used in the correlator element to obtain the final image.

\section{B. Shuttle Imaging Radar-A (SIR-A)}

The SIR-A was flown on the second flight of the Columbia in November 1981. The flight lasted for three days, and the radar acquired data during a total time period of $7-\frac{1}{2} \mathrm{~h}$. Even with a brief time period, images were acquired over selected regions in North, Central, and South America, Africa, Asia, and Australia. The total area covered was about 10 million square kilometers.

The SIR-A sensor is a modified, but similar, version of the Seasat sensor, and it used some of the residual Seasat hardware. The main differences in the SIR-A sensor were the following: a narrower bandwidth (6 $\mathrm{MHz}$ versus $18 \mathrm{MHz}$ for Seasat), a larger incidence angle $\left(50^{\circ}\right.$ versus $\left.20^{\circ}\right)$, a narrower swath (50 $\mathrm{km}$ versus $100 \mathrm{~km}$ ), a slightly lower resolution ( $38 \mathrm{~m}$ versus $25 \mathrm{~m}$ ), but a higher number of looks (6 versus 4 ). The SIR-A data were optically recorded on an on-board recorder. This allowed acquisition of data at any desired location around the world within the limitation of the orbital coverage. The "signal film" was retrieved after the Shuttle landing and was processed in an optical correlator (see Section V) to generate the twodimensional imagery. The orbital altitude was $245 \mathrm{~km}$. Fig. 15 shows photographs of the SIR-A sensor and antenna.

\section{Apollo Lunar Sounder (ALSE)}

The design of the ALSE was dominated by the primary objective of subsurface detection [67]. The potential of depth penetration of a radar wave is proportional to the wavelength of the wave. Since deep penetration was desired, the frequen- 


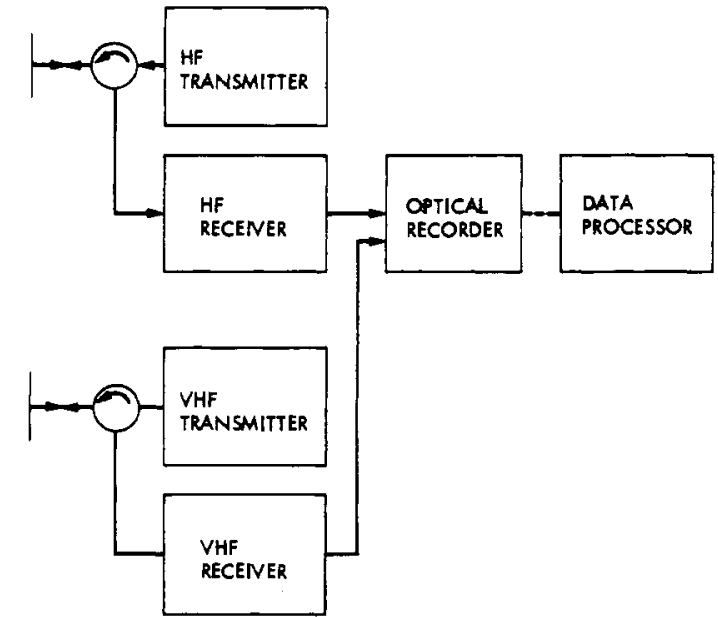

Fig. 16. Apollo Lunar Sounder Experiment system configuration.

TABLE III Lunar SOUNDer System Characteristics

\begin{tabular}{|c|c|c|c|}
\hline & \multicolumn{2}{|c|}{ HF Mode } & \multirow[t]{2}{*}{ VHF Mode } \\
\hline & RF1 & HF 2 & \\
\hline Wavelength (m) & 60 & 20 & 2 \\
\hline $\begin{array}{l}\text { Center frequency (MN2) } \\
\text { RF bandwidth (MHz) } \\
\text { Pulsewidth (Hs) } \\
\text { T1me-bandwidth product } \\
\text { Range resolution, Eree space (m) } \\
\text { Iransmitter peak power (W) } \\
\text { Irsnsmitter average power (W) } \\
\text { Effective antensa gain } \\
\text { (dB one-way, including efficiency) }\end{array}$ & $\begin{array}{l}5.256 \\
0.5333 \\
240 \\
128 \\
300 \\
130 \\
12.4 \\
-0.8\end{array}$ & $\begin{array}{l}15.8 \\
1.6 \\
80 \\
128 \\
100 \\
118 \\
3.7 \\
-0.7\end{array}$ & $\begin{array}{l}158 \\
16.0 \\
8.0 \\
128 \\
10 \\
95 \\
1.5 \\
+7.3\end{array}$ \\
\hline $\begin{array}{l}\text { Noise figure (dB) } \\
\text { Pulse repetition rate }\left(\mathrm{s}^{-1}\right) \\
\text { Recording duration (us) }\end{array}$ & $\begin{array}{l}11.4 \\
397 * \\
600\end{array}$ & $\begin{array}{l}11 ; ;^{4} \\
397^{*} \\
600\end{array}$ & $\begin{array}{l}10.0 \\
1984 \\
70\end{array}$ \\
\hline
\end{tabular}

Interlaced on HF1 and HF2.

cies used in the Apollo system were very low. The ALSE system was operated in three frequency ranges: $5 \mathrm{MHz}$ (HF-1), $15 \mathrm{MHz}$ (HF-2), and $150 \mathrm{MHz}$ (VHF). The two HF frequencies were operated simultaneously and were designed for deep sounding. The VHF system was designed for shallow sounding and for surface imagery. The flight ALSE system had four major hardware components (Fig. 16). At the heart of the system was the coherent SAR (CSAR) sensor containing the transmitters and receivers.

Separate transmit/receive antennas were provided for the HF and VHF ranges. The received signal was recorded by an optical recorder. The receiver also monitored the average reflected electromagnetic power and transmitted these data together with other engineering data by way of a $51.2-\mathrm{kbit} / \mathrm{s}$ telemetry channel. The average reflected power data were used for calibration purposes as well as for aid in data interpretation.

Since no internal access from the Command Module to the optical recorder was available, the photographic film on which the recorder stored the radar data was retrieved by an astronaut during an extra-vehicular activity (EVA); this was carried out toward the end of the Apollo 17 mission, during the transEarth phase. The basic parameters of the ALSE radar are given in Table III. Each of the three frequency bands utilizes a linear frequency-modulation (FM) transmitter pulse. An FM bandwidth of 10 percent of the carrier frequency is employed at each frequency band, as limited by the antenna subsystems. The linear FM pulse is generated by a voltage-controlled oscillator which, at the start of each pulse, is phase-locked to a stable local oscillator. The duration of the FM sweep in each system is set to maintain a time-bandwidth product of 128 . Upon reception of the echo, the signals are offset in frequency while maintaining phase coherence. The transmitted waveform envelope had a linear rise and fall for all three channels in order to limit the sidelobes caused by the Fresnel ripples.

Automatic gain control was incorporated at both HF and VHF. To avoid undue complexity in the data processing, the gain setting was allowed to change no more often than once every $30 \mathrm{~s}$ and then in discrete steps. During the flight of Apollo 17, the power return did follow predicted specular values on the average; however, significant variations in return power were observed over some regions of the lunar surface. The VHF subsystem incorporated a 12-dB increase in gain 13 $\mu s$ after arrival of the specular pulse. The initial gain of the radar system was set so that the specular pulse would have a received amplitude close to the saturation point of the optical recorder in order to achieve a wide dynamic range. However, shortly after arrival of the specular return, the level of the received signal was expected to drop significantly and rapidly as a result of weak diffuse off-nadir scattering. Consequently, additional gain was required in order to obtain a radar image of the lunar surface.

The HF-1 and HF-2 systems used a dipole antenna of 0.4 and 1.2 wavelengths, respectively. To achieve an antenna tipto-tip length of $24 \mathrm{~m}$, two extendable/retractable elements were required. A retracted element was spooled as two flat metallic ribbons, which, upon extension, curled together into a circular rod. To match the complex antenna impedance to the radar, an electrical network was constructed and mounted in the antenna housing. A hybrid unit mounted in the scientificinstrument module provided the power division and the appropriate electrical phase to feed the antenna elements as a dipole. To maintain low side lobes, the antennas were designed with extreme amplitude and phase linearity across the bandwidth. This was done at the expense of antenna efficiency; the minimum efficiency across the band was 65 percent for the HF-1 system and 43 percent for the HF-2 system.

The VHF antenna was designed to satisfy a linear amplitude and phase response as well as a directionality requirement. The antenna was a Yagi of five directors and one reflector. The Yagi, mounted at the rear of the service module, was erected by a spring mechanism. The antenna had a beamwidth of $70^{\circ}$ in the plane containing the antenna elements and a beamwidth of $50^{\circ}$ in the orthogonal plane. To suppress ambiguity between terrain returns on the left and right sides of the spacecraft in the imaging mode, the antenna axis was pointed $20^{\circ}$ off nadir.

The optical recorder was required to accept input data over the video bandwidths of the radar and to have adequate storage capacity to permit the radar to operate for nearly six orbits of the Moon. The video bandwidth is largest-16 $\mathrm{MHz}$-during VHF operation. The decision to use a CRT/film-type optical recorder was based both upon the ease with which such a recorder would meet the input and storage requirements, and upon the compatibility of the film-storage medium with coherent optical processing.

\section{Future Development}

A number of spaceborne SAR systems are presently under development or are being designed for flight in the 1980's. These include the German Spacelab radar system (flight in 
1983), the U.S. Shuttle Imaging Radar-B (in 1984), the Shuttle Active Microwave Experiment (SAMEX) radar (series of flights in 1986-1988), the Venus Radar Mapper (in 1988), and a number of free-flying systems for the late 1980's under study by the U.S., the European Space Agency (ESA), Japan, and Canada. The Shuttle-borne systems are expected to be more flexible with emphasis on scientific research, while the freeflying systems are planned for long-term semioperational observation with minimum variation in the operating parameters. The main emphasis in the next ten years is on multiparameter (i.e., multiple-frequency, multipolarization, variable observation geometry) and long-lifetime sensors. The Seasat and SIR-A sensors were all solid state. Going to higher frequencies ( $C$ - and $X$-band) requires the use of traveling-wave tubes (TWT's) or the development of high-frequency solidstate transmitters with peak-power capability between 2 and $10 \mathrm{~kW}$ and average transmitted power of a few hundred watts. New development in fieldeffect transistors (FET's) and electron-bombarded semiconductors (EBS's) should make it feasible to develop such high-power transmitters. TWT transmitters are capable of achieving the power requirements; however, they are less attractive than solid-state systems because of the need of high-voltage power supplies and their lower reliability for multiyear missions.

Another approach is to use a distributed array of low-power solid-state transmit-receive elements. These elements will be directly coupled to the antenna radiating elements. Such a distributed SAR approach will allow the radiation of a highpower signal, which is the superposition of the radiated signals from hundreds, or even thousands, of low-power elements. This technique is commonly used with ground-based radar systems.

The distributed SAR approach has another advantage. By using phase shifters, the beam can be controlled in both dimensions, allowing variable incidence angle and spotlight observation. It can also compensate for any mechanical distortions in the large SAR antenna, allowing a relaxation of the structural requirement which could be challenging at $X$-band frequencies.

\section{Digital Processing of Spaceborne SaR Data}

The SAR image formation process, as described in Section II, is to use coherent phase information in an array of radar echoes to synthesize an effective antenna aperture which is much larger than the size of the physical antenna. This approach enables high-spatial-resolution radar images to be attained with a practical-size antenna. Digital processing for SAR image formation involves sampled and quantized SAR echo data and represents a numerical evaluation of the synthetic-aperture beam-formation process. A large number of arithmetic computations are involved. The process, nevertheless, can be very accurate in representing the radiometric reflectivity of the target surface being imaged. The inherent flexibility in a sequential execution of digital SAR processing functions also allows users to extract special information such as Doppler spectra and pixel phases at various intermediate stages of the SAR correlation process. The added accuracy and flexibility of digital SAR processing relative to that of analog optical processing represent an appreciable advantage, which is considered rather significant as computer equipment is increasingly involved in performing quantitative analysis of remotely sensea data.

The arithmetic computation requirement for digital SAR image formation corresponds to the product of the number of computations involved to produce an output pixel and the pixel-throughput rate required for the system. For SAR image formation in real time, both the pixel rate and the per-pixel computation increase with the resolution capability. For a typical spaceborne SAR operating at $L$-band, such as Seasat SAR, the computation rate required for real-time processing exceeds $10^{9}$ operations/s. Conventional digital computers support a rate of approximately only $10^{6}$ operations/s. Such a high rate of computation indeed represents one major bottleneck in realizing an economical digital SAR processing system. For this reason, high-speed digital processors for airborne SAR sensors [50], [63] became possible only in the early- to mid1970 's with the availability of integrated circuits, which is two decades after the development of analog optical SAR processors.

The large number of arithmetic computations characterizes one part of the digital SAR correlation task. The numerical nature of the digital correlation process calls for the formulation of an accurate mathematical procedure, which is often referred to as the SAR correlation algorithm, to manipulate the sampled echo signals to accomplish the SAR correlation process. Design of this procedure is a two-step process. The first step involves an accurate modeling of the SAR response to a point target as well as a continuous field of reflecting targets. This modeling is required to formulate an inversion process and to provide all the necessary functions for reconstructing the target scene from the received echo signals. The model is also essential in evaluating the performance of a SAR System with respect to its attainable limit. The second step is to implement the processing functions of the SAR image reconstruction process in a clearly defined, mathematical computation procedure. Because digital processing is a sequential procedure, a computationally efficient algorithm often implies savings in computing time or hardware logics and may dictate the selection of a specialized hardware architecture. Besides the computational efficiency of the algorithm, the related costs of software and hardware implementation and the maintainability of the system are all of practical concern.

Digital processing for spaceborne SAR data concerns the general algorithmic and implementation issues described briefly above. Analysis of the response of a target to a spaceborne SAR has shown a number of differences relative to the treatment of a conventional airborne SAR. The focus of the first part of this section is thus on the computational algorithm for SAR image formation and the associated techniques in accommodating a number of peculiarities relating to data gathered by a spaceborne SAR. The different hardware architectures and several existing ground-based SAR processors and possible approaches for future development will be described in the later part of the section.

\section{A. Modeling of SAR Response to a Target Field}

Analysis of target response to an airborne SAR and the signal-processing principle was presented by Cutrona [26] and Leith [57]. As presented in Section II, spaceborne SAR involves a more complicated sensor-target relative-motion problem than the airborne SAR. A recent effort to provide a detailed modeling of target response to a spaceborne SAR sensor and the associated representations for signal processing was reported by Wu et al. [85]. Briefly, SAR echo response to a point target is a series of pulses, each of which is a replica of the original transmitted waveform, but is associated with a time or phase delay proportional to the round-trip distance to 
the target. This one-to-one correspondence between the echo time and phase delay is a result of the use of coherent wavetrain in generating the radar carrier and in demodulating it at reception. The ensemble of returned echo signals can be assembled into a rectangular format with spatial range delay, $r$, along one axis and the along-track or azimuth position, $x$, for the other axis. The echo response to a point target can therefore be written as

$$
h(x, r)=h_{1}(x, r) \otimes h_{2}(x, r)
$$

where $\otimes$ denotes a two-dimensional convolution, and

$$
\begin{aligned}
& h_{1}(x, r)=W(x) \exp \left(-j \frac{4 \pi r_{1}(x)}{\lambda} \delta\left(r-r_{1}(x)\right)\right) \\
& h_{2}(x, r)=\frac{2}{c V} \delta(x) A(r) \exp (-j \psi(r))
\end{aligned}
$$

where

$$
\begin{aligned}
& r_{1}(x) \text { round-trip distance from target to sensor at } x \\
& \psi(r) \text { phase encoding of radar transmitted pulses } \\
& W(x) \text { radar illumination at sensor position } x \\
& A(r) \text { amplitude waveform of the transmitted pulses } \\
& \delta(t) \quad \text { Dirac's delta function } \\
& V \quad \text { surface projection of the along-track speed of the } \\
& \text { sensor } \\
& \text { speed of light. }
\end{aligned}
$$

The function $h_{2}(x, r)$ is one dimensional, representing the waveform of the radar-transmitted pulse. Function $h_{1}$ is a curved response function, representing the locus of the target delay with a phase angle proportional to its range distance.

Radar echo return and reception is a physical process. The key issue in SAR imaging is the coherency of the carrier wave in the transmission-modulation and receiving-demodulation process, which results in a deterministic phase variation of the echo response from a point target. Equation (25) also shows that the transmitted waveform is preserved in the onedimensional time or range dimension; however, it is associated with a varying range and phase delay as the sensor changes its position in the $X$-dimension. This is clearly illustrated in Figs. 3-5.

For extended target with reflectivity $\sigma(x, r)$, the signal received by an idealized radar receiver represents a linear superposition of echoes from a continuous field $\sigma(x, r)$. The echo response $S(x, r)$ can be written as the following convolution:

$$
S(x, r)=\sigma(x, r) \otimes h(x, r) .
$$

Substituting (25) into the above, we have

$$
S(x, r)=\sigma(x, r) \otimes h_{1}(x, r) \otimes h_{2}(x, r) .
$$

Equation (29) describes the SAR echo response to an extended target $\sigma(x, r)$. The reconstruction of the scene can be accomplished by a two-step matched filtering involving the time-reversed range transmitted waveform $h_{2}$ and azimuth response $h_{1}$. Imaging radar systems often use a linear FMphase-encoded waveform; however, other coding schemes can also be used. For a linear FM waveform, deconvolution of $S(x, r)$ or $(29)$ with the reverse of the $h_{2}(x, r)$ function results in a sinc-function $(\sin a / a)$ type of response in the range dimention [20].

The waveform of $h_{1}$ depends mainly on the range delay function $r_{1}(x)$. For the Seasat SAR sensor in a near-circular orbit, the range or phase delay can be approximated by a second-order power series [85]. It is also clear that the $(x, r)$ coordinates are interchangeable with $(\tau, t)$ for the pair of alongtrack elapsed time, $\tau$, and range echo delay time, $t$, respectively. Using the notation $\tau$, the phase angle of $h_{1}(x, r)$ in (26) becomes

$$
\phi(\tau) \cong \phi(0)+2 \pi\left(f_{D} \tau+\frac{1}{2} \dot{f}_{D} \tau^{2}\right)
$$

where $f_{D}$ and $\dot{f}_{D}$ can be referred to as the Doppler center frequency and frequency rate, respectively. The phase delay $\phi(t)$ is proportional to the range delay $r_{1}(t)$ multiplied by $4 \pi$ over the radar wavelength. An additional factor of 2 accounts for the round-trip traveling of the radar transmitted wave. The polynominal expression in (30) is a convenient way to characterize the SAR Doppler phase response for digital SAR processing, and the functional expressions for $f_{D}$ and $\dot{f}_{D}$ factors in terms of sensor and target position and velocity vectors are derived by Wu et al. [85]. For Seasat SAR, the orbit geometry is such that magnitudes of the higher order terms in the series expansion of the phase function are indeed negligible.

By properly determining the numerical values of the Doppler parameters $f_{D}$ and $\dot{f}_{D}$ through the sensor orbit and attitude predicts and the SAR echo data, the function $h_{1}$ is now defined. A matched filtering of the range-compressed echo signals with the time-reversed $h_{1}$ completes the SAR image reconstruction process. Using $\hat{\sigma}(x, r)$ as the reconstructed scene, the expression of $\hat{\sigma}(x, r)$ is

$$
\hat{\sigma}(x, r)=\left[S(x, r) \otimes h_{2}^{-1}(x, r)\right] \otimes h_{1}^{-1}(x, r) .
$$

Substituting (29) into (31), $\hat{\sigma}(x, r)$ can be expressed as

$$
\widehat{\sigma}(x, r)=\sigma(x, r) \otimes\left[\left(h_{1} \times h_{1}^{-1}\right) \otimes\left(h_{2} \otimes h_{2}^{-1}\right)\right]
$$

where $h_{1}^{-1}$ denotes the time-reversed response of the $h_{1}$ function. The quantity given in the square bracket of (32) is now the response of the SAR-sensor system to a point target. As mentioned previously, a quadratic echo phase function results in a sinc type of response in the matched filtering process. Using linear FM pulse for radar transmission, the SAR sensor response to a point target resembles a two-dimensional sinc function. The half-power width of the main lobe is the resolution of the imagery. A detailed treatment of the resolution function in terms of the Doppler parameters is given by Wu et al. [85].

\section{B. SAR Correlation Algorithms and Correlator Architecture}

The previous subsection establishes the functional basis for reconstructing a scene from the SAR received echo signals. The algorithm development for digital SAR processing now deals with the formulation of an efficient computational procedure for implementing the matched filtering process given in (31). The compression of the range response $h_{2}$, which is a replica of the radar-transmitted waveform, is a onedimensional correlation operating on the sequence of sampled echo signals. This process is considered straightforward and is common practice in pulsed radars with amplitude or phase encoding of transmitted waveform. A common technique is to use SAW devices to perform the pulse compression. The focus of this section is on the compression of the SAR azimuth response $h_{1}$.

There are currently two categories of concepts in realizing the azimuth correlation function described in the previous subsection. The first one uses a cross-correlation process where a waveform which is an estimate of the target response 


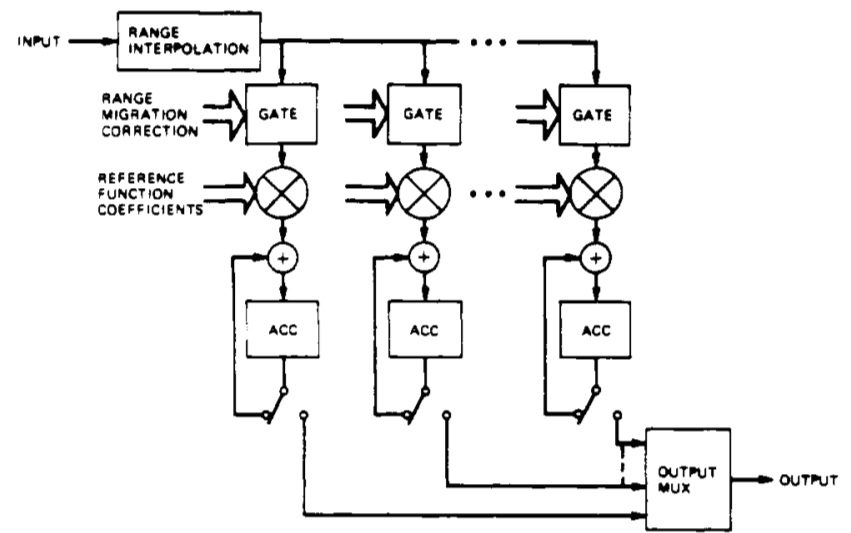

Fig. 17. Azimuth correlator architecture using distributed accumulator approach.

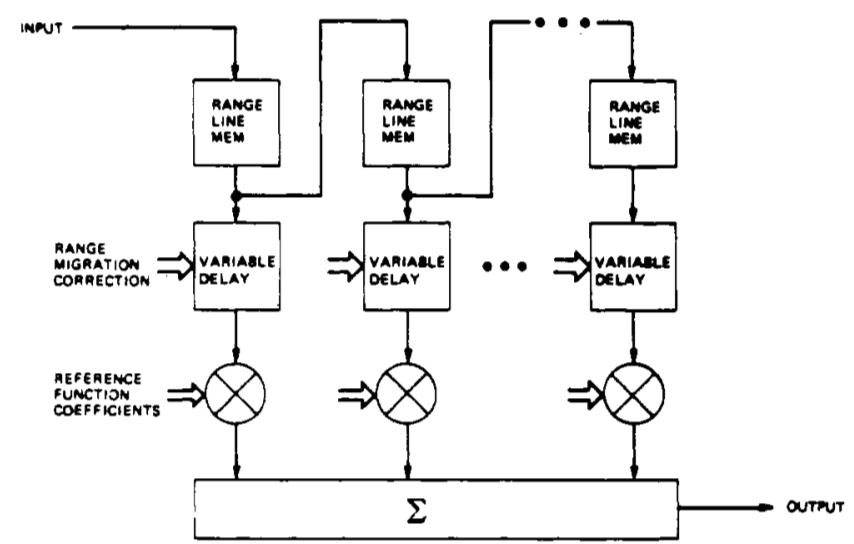

Fig. 18. Azimuth correlator architecture using tapped shift-register approach.

is generated to serve as the correlation reference function. The other category treats the SAR processing much as the signal processing for echo data received from a linear-phase-array radar. The ultimate long synthetic apertures are formed by several stages of array processing, each forming a longer aperture from outputs of shorter ones. In this approach, pulse compression is accomplished without the generation of the explicit target response waveform.

The correlation between signal data and reference can be accomplished by either a time-domain convolution process or a frequency-domain fast correlation approach. A usual timedomain convolver for one-dimensional signal correlation can assume either of two alternative forms: the distributed accumulator or the tapped-shift register, as shown in Figs. 17 and 18. For SAR data with severe range-curvature effect [69], both correlators illustrated in Figs. 17 and 18 incorporate appropriate compensation to accommodate such a curved two-dimensional SAR response. The configuration in Fig. 17 incorporates gating control to select the appropriate data samples. The configuration in Fig. 18 incorporates extra shift-register cells and control to enable selection of the appropriate sets of data samples. The two configurations shown in Fig. 18 exhibit the following differences: 1) the dynamic range requirement for the accumulator memory cells is greater than that for the tapped shift-register data sample memory cells; and 2) the accumulator approach involves the passing of coefficients from channel to channel whereas the shift-register approach involves the passing of radar data

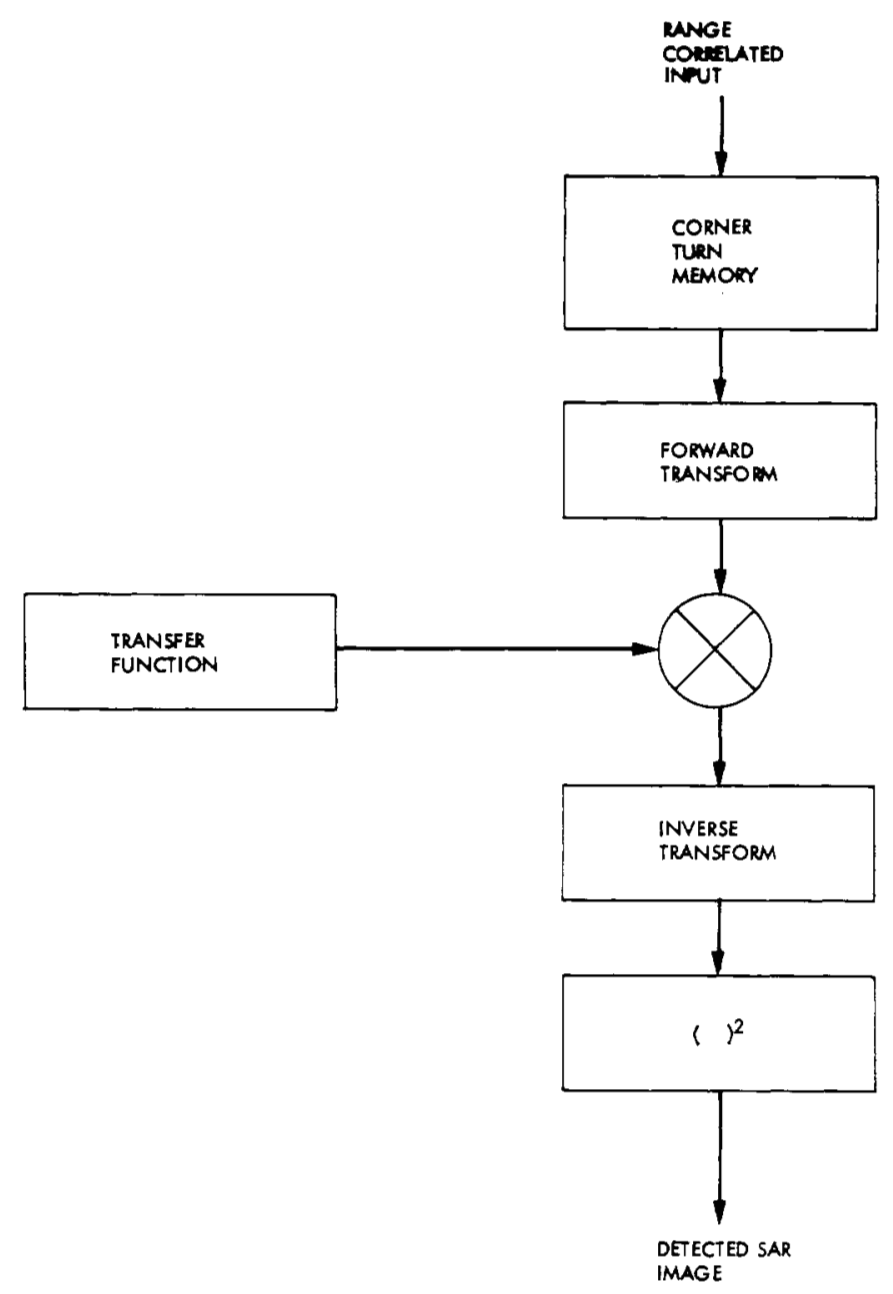

Fig. 19. Frequency-domain convolver for SAR azimuth correlation.

samples from channel to channel. Considerations on largescale integration (LSI) implementation of such a correlator were discussed by Tyree [79].

The SAR correlation can also use the fast Fourier transform (FFT) as a means of reducing the number of arithmetic operations relative to the direct time-domain approach. After the range correlation is accomplished, a range "corner-turn" operation is done which changes sequential data access from the range dimension to the azimuth dimension. A functional block diagram of this approach is shown in Fig. 19. Note that the transform involved can be any efficient linear transformation that exhibits the convolutional property. Applications of the FFT for SAR correlation were reported by $\mathrm{Wu}$ [82], Cumming [22], and Vant [81], and the polynominal transform by Truong [78].

The other approach to SAR processing, which does not involve azimuth response waveform in an explicit manner, generally makes use of the fact that the Doppler response given in (30) is quadratic. Synthetic-aperture processing thus resembles the compression of such a linear FM or chirp signal. In this section, we briefly describe two such digital SARprocessing algorithms.

A time derivative of the phase history in (30) provides a linear frequency and time relationship of the target response. The Doppler frequency response versus time for multiple targets which are separated in azimuth but are at the same range is shown in Fig. 20(a). The finite response in time and 


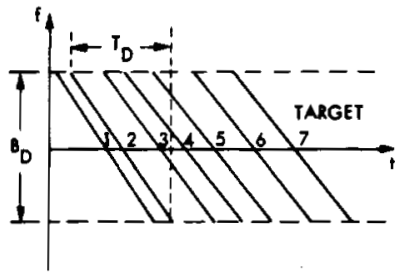

(a)

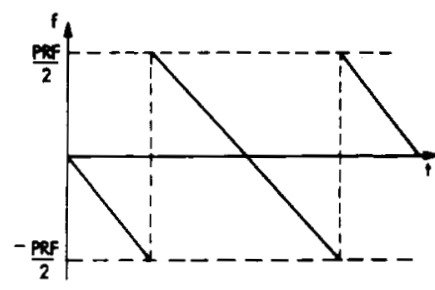

(b)

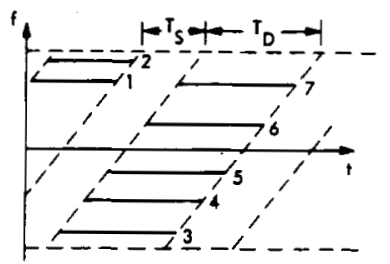

(c)

Fig. 20. SAR processing by sweep mixing and frequency analysis: (a) Doppler responses to multiple targets in azimuth; (b) sawtooth sweep to be mixed with the signal in (a); (c) frequency responses after mixing.

frequency for each point reflects the finite duration that a target is illuminated by the main radar beam. The finite time duration and Doppler bandwidth are indicated by $T_{D}$ and $B_{D}$, respectively, in the figure. The composite signal return when mixed by a coherent frequency-sweep waveform, which is shown in Fig. 20(b), results in a signal represented in Fig. 20(c). SAR responses to targets at different locations are now resolved into different frequency and time intervals. The frequency sweep covers a range over the radar PRF, which is always designed to be greater than the Doppler bandwidth $B_{D}$. The oversampling margin, which provides a corresponding time separation $T_{S}$ between two responses of the same frequency, allows unambiguous resolution of such targets by selecting a proper data block for the frequency analysis. The block size must be between $T_{D}$ and the sum of $T_{S}$ and $T_{D}$. Using the maximum allowable size, a fraction $T_{S} /\left(T_{S}+T_{D}\right)$ of the frequency-transformed results is valid. Overlapped blocks are then necessary to provide continuous output in the azimuth dimension. A two-dimensional radar map is produced by repeating this process for samples at different range positions. The technique described here is often referred to as the "deramp" approach and was reported by Brown et al. [15].

One method, which is often referred to as the step-transform [59], or the subarray [80], can be used to simplify the arithmetic operations of the deramp method described above. Instead of using a block size which is greater than the synthetic-aperture length $T_{D}$, the procedure is multistage and operates on a much smaller block of data at each stage. The idea is shown in Fig. 21(a). Small data blocks and segmented sweeps are used. The frequency resolution which is directly related to the spatial resolution of targets is very coarse due to the small number of signal samples contained in a data block

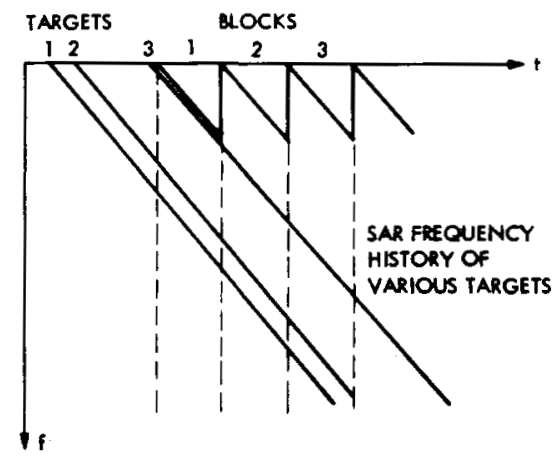

(a)

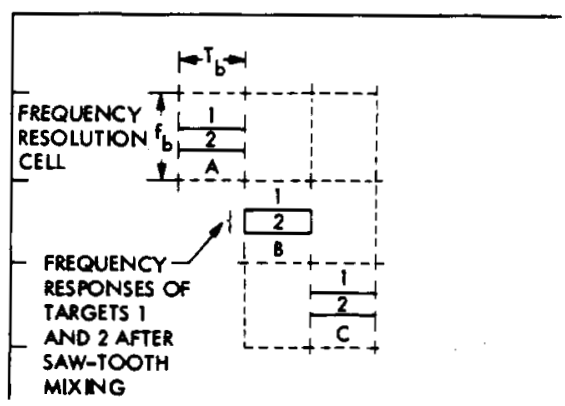

(b)

Fig. 21. Illustration of the time and Doppler frequency relationship of the step transform approach: (a) auto mixing with segmented sawtooth sweeps; (b) time and frequency ranges for samples $A, B$, and $C$.

(frequency resolution is PRF/N in the first step of frequency analysis, where $N$ is the size of block). Fine frequency and target resolution can be obtained by performing another level of analysis on data samples from different blocks but carrying the coherent Doppler information of the same target area (see Fig. 21). Fig. 21(a) shows the frequency histories of several targets and segmented sawtooth sweeps. The points used for the second step of analysis are labeled $A, B$, and $C$ in Fig. 21(b). Special conditions must be met for a good timeand-frequency correspondence to perform frequency transform and analysis in the second step of operation. The dashed grid in Fig. 21(b) provides that the fine frequency of a given target, e.g., target 1 or 2 , is fixed over the blocks. This condition is met when there are equal numbers of blocks over the full synthetic aperture and numbers of frequency-resolution elements over the maximum Doppler bandwidth. This implies that the size of the time block equals the square root of the product of the full aperture duration and the corresponding Doppler bandwidth [59]. Note that points $A, B$, and $C$ in Fig. 21(b) can be in the same band of frequency resolution rather than in the stepped bands as shown, if a continuous long sweep such as the one in Figs. 20(b) is applied.

The subarray type of processing approach reported by Van de Lindt [80] is similar in principle to the multistaged approach shown above. The interpretation is viewed through the array-antenna synthesizing. At each stage of operation, a longer antenna aperture is synthesized based on the shorter ones produced from the previous stage. It is required that the arrays in order to be synthesized in a higher level must all be pointing toward the same patch of target area in both the azimuth and range distance directions.

The approaches described above differ in the number of 
arithmetic computations and the complexity in processing control. In general, for the case of large time-bandwidth product, the time-domain correlation is associated with the highest degree of arithmetic computation and parallelism. The frequency-domain fast correlation has potentially the simplest control function for high-resolution processing. The multistage frequency analysis or subarray approach involves the least amount of arithmetic computation. The processing complexity issues are discussed in the next subsection.

\section{Processor Complexity Issues}

The general correlation and architectural concepts for digital SAR processing have been described above. A major difficulty in realizing a digital SAR processing system for high-resolution and high-swath imaging is mainly associated with the requirement for a very large amount of data memory for line storage/ corner-turn or accumulation and a very-high-speed arithmetic computation requirement. The curvature effect is, in general, more severe for a spaceborne SAR than an airborne SAR because of the larger value of $V^{2} / R$, which is equivalent to the centrifugal acceleration of the sensor relative to the target. The range curvature requires that the azimuth response $h_{1}$ must be treated as a two-dimensional function.

The variation of Doppler parameters is caused by the changing of the tangential velocity of targets on Earth or planetary surfaces due to the rotation of the planet. The change of tangential velocity affects both the Doppler variation over the swath because of the varying angle of incidence over the swath and the variation over different orbit positions or latitudes where the target surface speed is a function of latitude. The rate of change of Doppler parameters is inversely proportional to the radar frequency. Rapid change of correlation reference functions is therefore required in order to obtain high-quality SAR imagery.

The large number of memory storage and arithmetic units, and the added procedures to accommodate the severe-range curvature and Doppler-variation effects, both significantly impact the complexity of a digital SAR processor. A brief and simplified assessment of the arithmetic calculations requirement is given here.

The complexity of a specific digital SAR processing approach is characterized by arithmetic complexity and control-function complexity. The arithmetic complexity depends on a number of factors, the most predominant being the throughput rate, the pulse-compression ratio, and the number of looks. The arithmetic-processing rate can be measured by the product of the pixel rate at the output of the processor and the number of arithmetic operations required to produce each pixel. The pulse-compression factor of SAR azimuth correlation is equal to the ratio of the synthetic-aperture length to the corresponding azimuth resolution. This is equal to the number of complex arithmetic operations $K_{t}$

$$
K_{t}=\frac{\mathcal{L}}{r_{a}}=\lambda R / 2\left(r_{a}\right)^{2}
$$

where $\mathcal{L}$ is the azimuth footprinted on the surface. For a nearoptimal SAR system, the echo window is nearly equal to the separation between pulses, and $r_{a}$ is equal to half the physical aperture. The pixel rate $P$ is very close to the bandwidth $B$ of the radar transmitted pulses. Thus

$$
P \simeq B=\frac{c}{2 r_{r} \sin \theta}
$$

The arithmetic-complexity measure $A_{T}$ is the product of $K_{T}$ and $P$, and is given by

$$
A_{T}=\frac{\lambda R c}{4 r_{r}\left(r_{a}\right)^{2} \sin \theta} .
$$

For the frequency-domain processing approach using the FFT multistage processing, the number of arithmetic operations per pixel is approximated by

$$
K_{f}=\log _{2} K_{t}
$$

and the complexity measure $A_{f}$ is given by

$$
A_{f}=\frac{c}{2 r_{r} \sin \theta} \log _{2}\left|\frac{\lambda R}{2 r_{a}^{2}}\right| .
$$

Equations (35) and (37) are useful for a quick assessment of the arithmetic complexity of the processor for various SAR systems.

The control-function complexity is not easily quantifiable. In general, the control complexity increases as the systemperformance requirements (resolution, sidelobe levels, etc.) become more stringent. The SAR processor must include a controller which determines accurately the parameters required for processing. These include exact description of the range history between the radar and the target, which requires precise knowledge of the orbital and attitude characteristics, as well as the surface curvature and surface velocity at the point of observation. Fig. 22 summarizes the control functions. Predictions of SAR Doppler processing parameters can be obtained from the spacecraft state vectors, which are part of the ephemeris. Refinement of the processing parameters is then obtained by a feedback loop (clutterlock and autofocusing). Rapid updating of the azimuth correlation reference functions and range-curvature compensation coefficient increases the complexity of the overall processor.

\section{Clutterlock and Autofocusing}

Clutterlock and autofocusing refer, respectively, to the estimation of the instantaneous Doppler frequency $f_{D}$ and Doppler frequency $\dot{f}_{D}$ at the center of antenna azimuth beam based on the SAR received echo data. These two parameters define the basic response of the SAR system to a point-target according to (30), and are crucial in computing the SAR correlator response to obtain high-quality SAR imagery. Since SAR response waveform based on a quadratic phase function which has a linear frequency response over the time span represents the typical linear FM waveform, compression of such waveform, and the distortion due to mismatch in the frequency and rate coefficients are well known in radar signal processing, e.g., Cook [20]. Quantitatively, a mismatch in the center frequencies $f_{D}$ of the echo response and the correlator parameter results in an apparent shift in the target position as well as a loss in the output SNR; and a mismatch in $f_{D}$ results in a degraded radar resolution.

These Doppler parameters are solely determined by the relative-motion characteristics between the SAR sensor and the targets. They can be estimated accurately provided that precision orbit and attitude data of the spacecraft are given. The clutterlock and autofocusing are used primarily in cases where the available orbit and attitude predicts are not accurate enough to maintain a reference phase function that meets the requirements on image quality.

The clutterlock techniques in general make use of the fact 


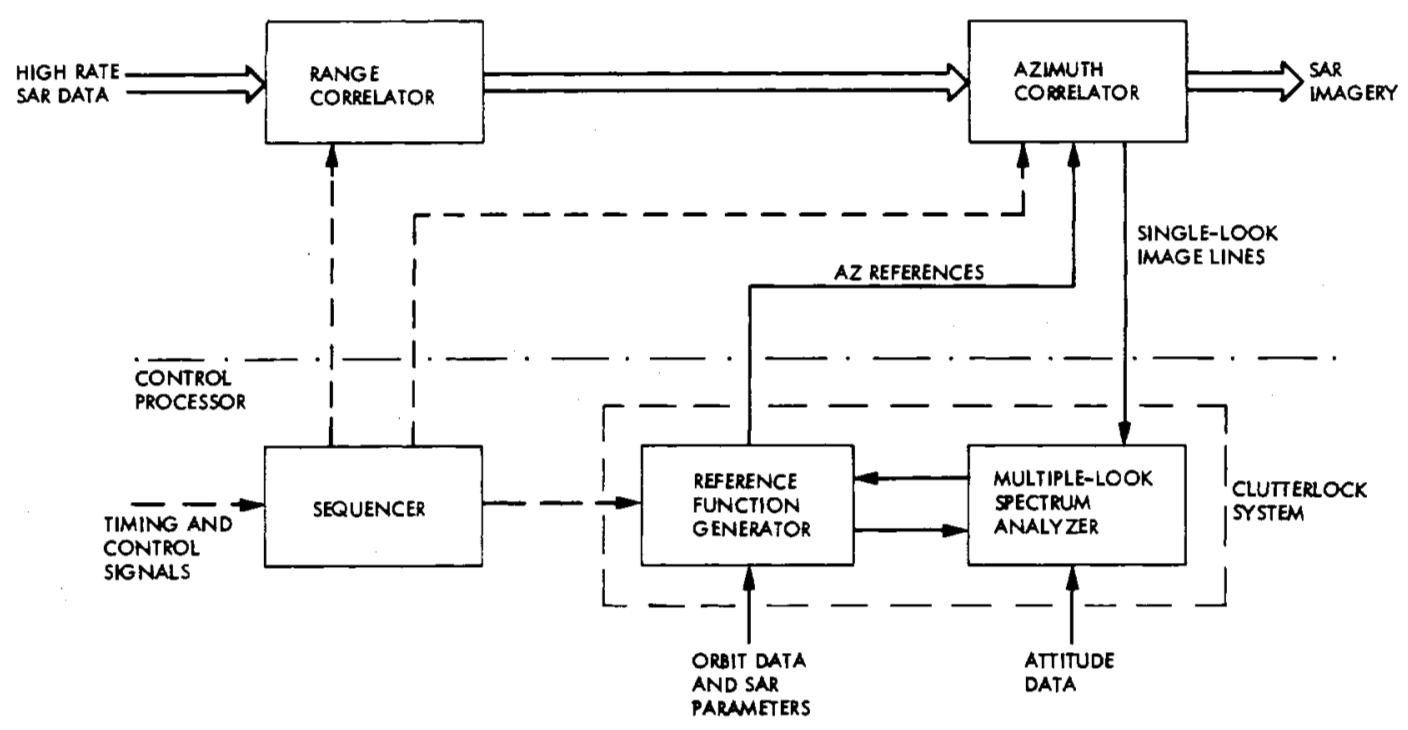

Fig. 22. Real-time SAR processor control block diagram.

that the SAR antenna response in azimuth is nearly identical to the SAR Doppler spectral response due to a one-to-one relationship between the radar perspective angle in the alongtrack direction and the instantaneous Doppler frequency of a target. The Doppler frequency at the center of the antenna along-track beamwidth can be obtained by evaluating the Doppler spectral pattern of the echo. This method is subject to error because the Doppler spectral pattern obtained over a short period of time can be affected by the presence of strong targets in the leading or trailing part of the antenna beam. More sophisticated approaches based on the response from the same target area can attain an accuracy on Doppler frequency estimate over a finite target area to within a few hertz ([83], [24]).

SAR systems are often designed to have a Doppler bandwidth measured over half-power points within 80 percent of the radar PRF. Since the Doppler spectral pattern varies smoothly, an estimation accuracy of the Doppler center frequency to within a few percents of the PRF is adequate to meet most of the image-quality requirements. Accurate estimates are most promising for possible information-extraction applications, such as the determination of the SAR platform attitude from SAR data [84], and ocean currents.

The autofocusing technique estimtes the Doppler frequency rate from SAR echo data. The mismatch in $\dot{f}_{D}$ induces image blurring that can be detected by measuring the effective spatial bandwidth of the processed image. In a multiple-look SAR correlator, the mismatch in $\dot{f}_{D}$ can also be detected from the misregistration of the different single-look scenes. As a result of the linear FM waveform, it is easily derived that the amount of misregistration between looks is linearly proportional to the mismatch of $\dot{f_{D}}$. This method through multiple-look registration is very effective but is subject to the existence of clearly discernible targets to obtain the registration measures [63].

\section{E. Existing Ground-Based Digital Processors for Spaceborne SAR's}

Seasat SAR has been the only available spaceborne highresolution imaging radar for remote-sensing applications with digital data handling. The tremendous interest in obtaining high-quality SAR imagery and in quantitative data analysis using computers prompted the development of digital processors for producing Seasat SAR imagery. Digital SAR correlators capable of producing imagery at a near-real-time dataacquisition rate were not possible due to the need of a high-throughput processor that met the stringent computation and control requirements discussed before. Software-based SAR processors using programmable peripheral array processors were first developed in the U.S. (Jet Propulsion Laboratory) and Canada (MacDonald, Detwiller and Associates), and then in Europe (Royal Air Force, England) and Japan (Nippon and Mitsubishi). A frequency-domain fast-correlation algorithm was the main approach in compressing the dispersed range and azimuth responses into a narrow pulse. The commercially available peripheral array processors such as the AP-120B manufactured by the Floating Point System are used in computing the FFT and other vector processing at a much faster rate than that of a conventional general-purpose computer. The commercial array processors in general have an adder and a multiplier in parallel. The hardware is designed to allow pipelined operations in those multipliers and adders as well as in other storage memory units. For vector processing where the procedure is highly repetitive and the data storage in memory is in form or arrays, this parallel and pipelined execution of data access and arithmetic units at a rate of one set of such operations per machine-clock period allows a very high-throughput capability. A review of those array processors for vector data processing is reported in a special issue of the IEEE COMPUTER magazine (Sept. 1981).

The digital correlator currently existing at JPL applies three parallel AP-120B array processors in performing the FFT fast correlation to produce Seasat SAR imagery [86]. This system is called the Interim Digital SAR Processor (IDP). The IDP throughput is currently one five-hundredths of the real-time data-acquisition rate. Noting the fact that Seasat SAR calls for a compression ratio (number of samples required to be coherently integrated to compress the pulse and produce one output sample) of approximately 700 in range correlation and 200 per each of the four looks in azimuth to produce a final pixel, and the pixel rate in real-time acquisition is approxi- 
mately 2.4 million pixels/s, the current system is, nevertheless, still a very cost-effective approach. The IDP System currently produces a digital $100-\mathrm{km} \times 100-\mathrm{km}$ Seasat SAR frame in about $2 \mathrm{~h}$. Some of the digital products are shown in this paper.

The Canadian processor, developed by MacDonald Detwiller and Associates (MDA) is described by Bennett [8]. The FFT fast correlation in conjunction with an AP-120B array processor is applied in the MDA system. Software implementations of frequency-domain fast correlation were also made on the CRAY-1 computer [65] and other general-purpose computers [9], [64], [58]. A summary of various SAR processor development was reported by Guignard [43].

\section{F. Future Developments}

Digital data processing for spaceborne SAR sensors generally requires a large number of arithmetic computations and very involved processing control functions to produce a meaningful size of image. Future spaceborne SAR flights certainly require a fast throughput and accurate processing system to support experiments aimed at applications for real-time environment observation, especially ocean and ice. A real-time digital SAR processor thus is the development goal, and is being pursued in the U.S., Canada, the European Space Community, and Japan.

SAR processing algorithms and knowledge of peculiarities in the control functions for spaceborne SAR data are much better established now than prior to the launch of Seasat SAR. Implementation of a real-time SAR processor involves tradeoffs between cost and functional flexibility, arithmetic accuracy, and other performance requirements. We expect the cost-effectiveness of a digital SAR processor will continuously benefit from advancement in electronics technology. Specific areas that are applicable to future SAR processor development include: the computer-aided design (CAD) techniques for custom very-large-scale integrated circuits (VLSI) and gatearray logics, faster and more compact memory devices as well as peripheral array processors, distributed processing techniques, etc. This list covers a wide spectrum of advanced electronics technology development, even though the quantity of SAR processors that may be manufactured is quite limited. Real-time digital SAR processor development would definitely contribute in the area of electronics applications and distributed system techniques. A discussion on the trend of radar-signal processing development is more thoroughly presented by Brookner [13].

Development in the next decade may still be focused on ground-based SAR processors. A real-time spacecraft onboard SAR processor is feasible provided that 1) the processing operations can be handled by a system within the speed, volume, and power constraints; and 2) the required applications and the range of flexibility are well defined.

\section{Optical Processing of Spaceborne SaR Data}

Optical techniques for processing SAR data were developed primarily in the 1960's. Since that time, there have been no major developments of new techniques. One reason for the lack of further development has been the emphasis on digital techniques, with the inherent advantages of accuracy and adaptability. Another reason is that the state of the art in the optical technology used in conventional processors has not progressed significantly, and major improvements did not
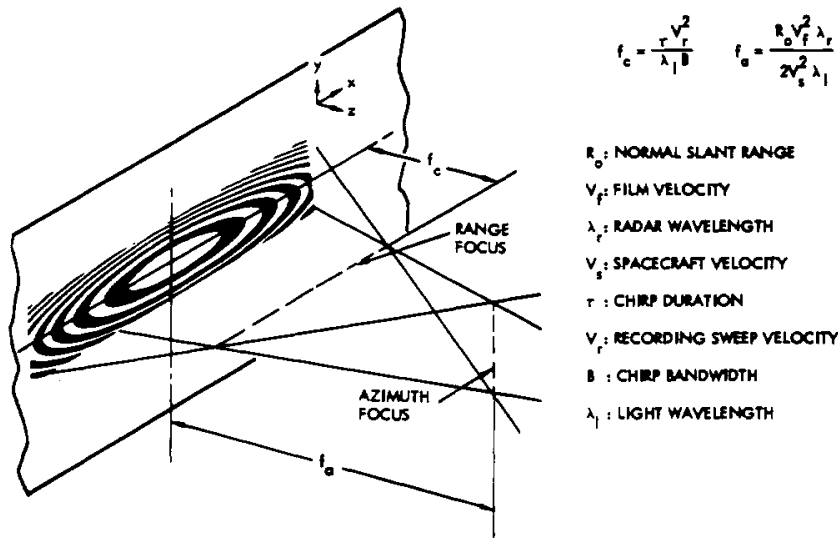

R: NOEMUL SLANT RANGE

$V_{f}$ FILAT VELOCTY

A: RODA WAVELENGTH

$V_{\text {: SPACECANT VELOCTY }}$

$T:$ CHIEP DURATION

$V_{r}$ : RECORDHNG SWEP VELOCTY

D: CHIR BaNowioth

$\lambda_{1}$ : LKGHT WAVELENGTH

Fig. 23. Focal properties of a point-target phase history.

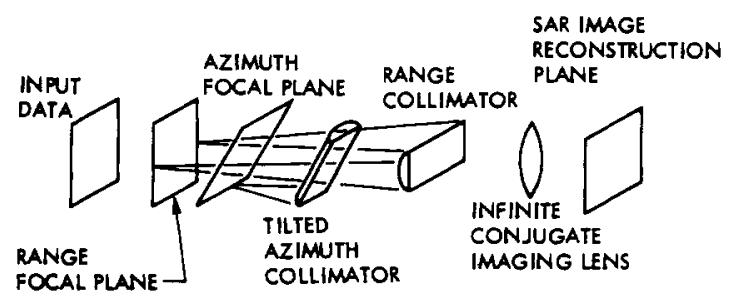

Fig. 24. Configuration of a simplified optical correlator system.

appear feasible. However, recent interest in the applications of spaceborne SAR, has led to renewed emphasis on SAR data processor development, both digital and optical. The primary effort in digital techniques is centered on throughput improvement, while the emphasis in optical systems is the reduction of size, weight, and power consumption for remote-site or onboard processing applications.

\section{A. Conventional Optical Processing}

The phase history generated by a point target (Figs. 3-5) is similar to a Fresnel-zone plate. This fact makes SAR data natural candidates for optical processing. A basic introduction to optical processing of SAR data is given by Goodman [41]; see also [51]. Since the phase function is quadratic in both dimensions, it diffracts a coherent plane wave, illuminating it into a converging beam that will focus at a point. Unfortunately, the converging wave will come to a focus at different distances from the zone plate for the range and azimuth directions. These distances are referred to as the range and azimuth focal lengths, respectively. They are shown graphically in Fig. 23, and they are expressed in terms of the radar system parameters.

An important point to note is the dependence of the azimuth focal length on range. Not only is the azimuth focal length different from the range focal length, but it varies linearly with range dimension. This linear variation can be compensated for by using a conical lens. Manufacturing such lenses is difficult, however, and a cylindrical lens rotated about the horizontal axis leads to the same compensation. One of the simpler configurations for an optical correlator is shown in Fig. 24. A cylindrical lens in range is placed so that its input focal plane occurs at the zone-plate range focus, therebv collimating the wavefronts of all targets. A second cylindrical lens is used in a similar manner in azimuth. The lens is rotated so that its input 
focal plane coincides with the tilted focal plane of the azimuth phase histories. Now both dimensions are collimated, i.e., imaged at infinity, and a spherical lens placed behind the cylinders will focus both dimensions in its back focal plane.

\section{B. Optical Correlator System Description}

The configuration of the Seasat and SIR-A processors with the tilted-lens mode of operation is shown in Fig. 25. A confocal, spherical lens pair, called the range telescope, images the range and azimuth focal planes. A three-lens cylindrical telescope demagnifies the azimuth dimension. An auxiliary lens pair composed of a tilted azimuth cylinder and a vertical azimuth cylinder are used at the output of the azimuth telescope to bring the azimuth image into coincident focus with the range image. Frequency filtering and range-migration correction are done at the back focal plane of the first spherical lens by a combination of cylindrical lenses. A relay lens magnifies an intermediate image onto the output film drive. The spatial filter is composed of a microscope objective lens and a pinhole. The pinhole is located at the back focal point of the objective, blocking out spatial noise in the laser beam. Thus a "clean" diverging spherical wave emerges from the filter.

The collimator is composed of two elements. The first element is a convex-concave negative lens and the second element is a plano-convex lens. When the aperture of the input lens is illuminated by the diverging wave from the filter, the output will be a plane wave which is then reflected through a $90^{\circ}$ bend by mirror $M_{1}$ toward mirror $M_{2}$, the first element of the primary optical system. Mirror $M_{2}$ bends the beam through another $90^{\circ}$ angle and can be adjusted to provide an offset illumination angle in range and/or azimuth. It is desirable to have the center frequency of the spatial frequency band in both dimensions aligned with the optical axis to insure optimum use of the lens apertures. Thus if a signal film has equal positive and negative frequencies in both range and azimuth, the center of the band is zero frequency in both dimensions. Hence, the film should be illuminated with an on-axis beam. However, if the data have equal positive and negative frequencies in azimuth but run from 0 to some positive frequency in range, a range offset would be used. This case is the normal mode of operation.

The input film drive transports the signal film to be processed. The optical axis position of the film drive is determined by the range focal length of the signal film. The range telescope is a confocal spherical lens pair. Spatial frequency spectrum of the data occurs at the back focal plane. An expanded view of the frequency plane optics is shown in Fig. 26.

The frequency plane filter is used to block all light outside the bandwidth of the data to be processed. Two pairs of knife edges in range and azimuth are adjusted to the rectangular aperture corresponding to the range and azimuth bandwidth. Since illumination offset is used to bring the center of the bandwidth along the optical axis, the rectangle will, in general, be centered about the optical axis.

The shift lens, also located at the frequency plane, is a cylindrical lens with power in the azimuth direction. It is used for films with azimuth focal lengths that are too long to allow processing (without the shift lens). The shift lens does not reduce the tilt angle of the azimuth focal plane but translates it in the negative direction (opposite the direction of light) along the optical axis. Rotation of the shift lens about the optical axis introduces positive or negative range walk depend-

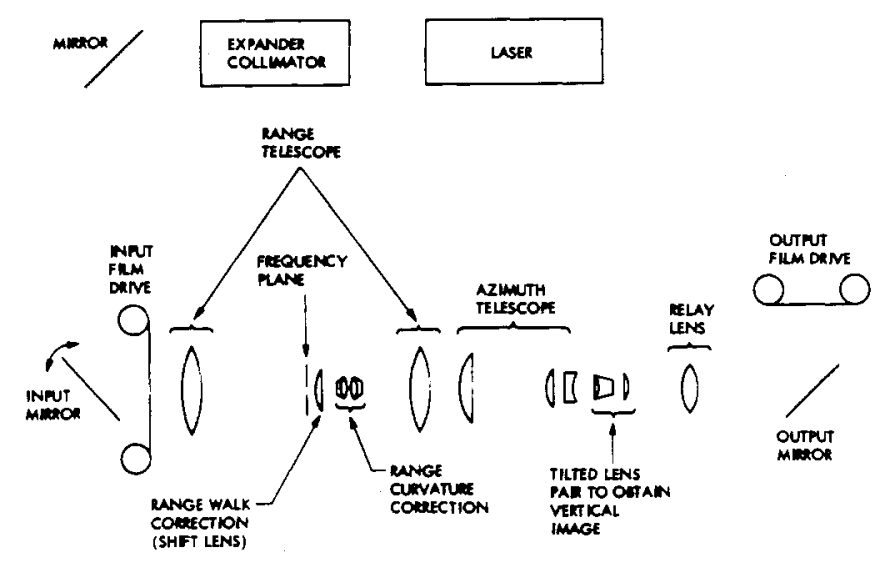

Fig. 25. Details of the Seasat SAR and SIR-A optical correlator.

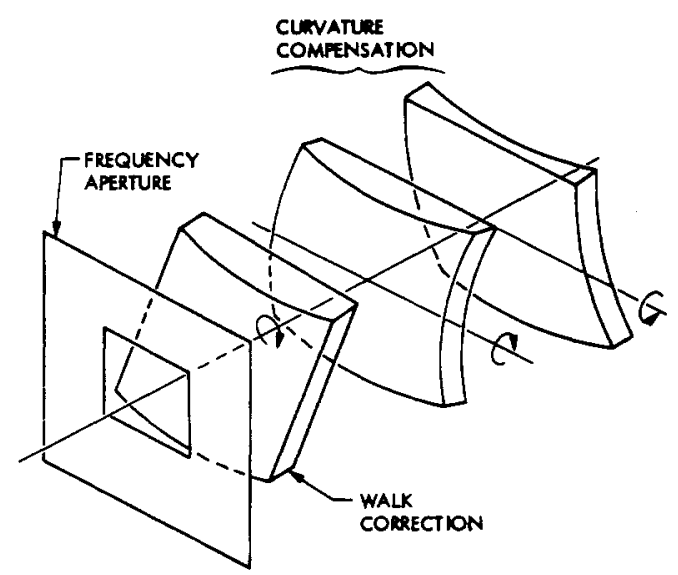

Fig. 26. Frequency plane optics showing the frequency filter, the curvature compensation, and the walk correction elements.

ing on the direction of rotation. This adjustment is used to remove range walk. A positive and negative azimuth cylindrical lens pair is located immediately after the shift lens. The lenses will introduce positive or negative range curvature when rotated in opposite directions about the horizontal axis perpendicular to the optical axis. This adjustment is used to remove range curvature.

The second range telescope lens is identical to the first with its orientation with respect to light direction reversed. It is located so that its input focal plane coincides with the output focal plane of the first lens. This combination forms a telescope with one-to-one magnification.

The azimuth telescope is a three-lens cylindrical telescope used to demagnify the azimuth image. The azimuth image will usually occur within a few inches of the output focal plane of the last lens. The demagnification setting is adjusted so that the azimuth scale factor of the image center matches the range scale factor.

A cylindrical lens pair is located immediately after the azimuth telescope. The first is tilted about the horizontal through its center perpendicular to the optical axis. The angle corresponds to the angle of the azimuth focal plane. This lens collimates the azimuth phase histories, removing the focallength variation with range. The second lens refocuses the phase histories to its back focal plane which is coincident with the range focus. This plane contains an intermediate image of the SAR focused data. 
A relay lens is used to magnify the intermediate image onto the output film drive. The magnification factor is normally set so that the output image will have a specified scale factor.

\section{Special Considerations for Spaceborne SAR Data Processing}

1) Extensive Phase Histories in Range and Azimuth: In order to reduce peak power, spaceborne radars usually transmit frequency-modulated long pulses, resulting in a high timebandwidth product of the chirp to be recorded. Since the data record will eventually be recorded in a two-dimensional raster format, a high sweep linearity will be required in the range dimension. The accuracy is approximately the reciprocal of the time-bandwidth product. In the case of Seasat, the accuracy required was approximately 1 part in 1000 . Only the highest precision type of recorder can normally achieve such accuracy. The CRT type of recorder used for Seasat required the addition of a digitally controlled correction signal to the basic sweep signal.

The large slant range at which spaceborne SAR's operate gives rise to a large azimuth phase history with a high timebandwidth product. As in the case of the range dimension, the azimuth dimension must be recorded linearly to an accuracy approximately equal to the reciprocal of the time-bandwidth product. In the case of Seasat, this number was about 1 part in 4000 for a single-look resolution of $6 \mathrm{~m}$.

The linearity of the recording in azimuth must be such that the focal length for a given slant range remains constant as the spacecraft velocity changes. Since the orbit is circular, the radar is accelerating toward the target as it passes over it. This causes the effective velocity to be lower than the actual tangential or orbital velocity. It can be shown that the effective velocity is proportional to the orbital velocity according to the relationship

$$
V_{\text {eff }}=\sqrt{\frac{\text { Earth radius }}{\text { orbit radius }}} \times V_{\text {orb }} .
$$

This poses an interesting problem when we try to maintain accurate focus simultaneously with a constant azimuth scale factor since the ground velocity (of the antenna footprint)

$$
V_{\text {ground }}=\frac{\text { Earth radius }}{\text { orbit radius }} \times V_{\text {orb }} \text {. }
$$

The differences in focus and scale factor are usually small enough to ignore. The long phase histories have long focal lengths in range and azimuth, requiring unreasonably long optical path lengths or the addition of shift lenses. A combination range- and azimuth-shift lens was required for Seasat to reduce the optical path lengths to an acceptable level. The shift lens was also used as the range-walk correction lens. Corrections for severe range walk cause focal shifts which must be compensated for.

The combination of large phase histories and large numbers of range-resolution cells leads to a requirement for large optical apertures at high resolution. The number of input data points in range was approximately 13000 for Seasat, several times that of the best CRT recording systems. Such resolutions are obtainable by laser-beam recording techniques. However, not many coherent optical correlators are capable of processing such a high space-bandwidth product $(6000)$. The system for processing Seasat data described earlier does have a 10000 space-bandwidth product in each dimension, but the recorder used was a CRT recorder with only about a 1500 product.

2) Range Curvature and Walk Corrections: While only veryhigh-resolution or long-wavelength airborne SAR systems require range-migration (combination of curvature and walk) compensation, spaceborne SAR systems generally will because of the long slant range involved. The Seasat SAR range curvature was on the order of 5 resolution cells, and the walk was sometimes over 100 resolution cells. The basic technique for correction of range curvature and range walk was described earlier. Since the relative change in slant range for spaceborne SAR systems is usually small, a single curvature correction is usually accurate for a near-circular orbit. However, range-walk compensation may have to be updated several times within the range swath. This update rate will increase with attitude errors, resolution improvements, and slant-range increase.

3) Doppler Tracking: Since spacecraft velocities are so great compared to aircraft velocities, it is desirable to use narrower beamwidths to reduce the Doppler bandwidth and hence the PRF. This narrowing of beam usually necessitates some type of Doppler tracking system since attitude changes and planet rotation will cause Doppler shifts that are significant when compared to the bandwidth. At higher frequencies, such as $X$-band, the Doppler shifts may often be an order of magnitude greater than the bandwidth, or many times the PRF. While Doppler tracking in an optical system is conceptually easy, the system becomes very complicated when trying to maintain accurate focus and location.

The actual Doppler spectrum is displayed in the frequency domain of the range telescope. Energy centroid detectors or a pair of detectors placed on either side of Doppler centroid peak may be used to determine the center, or at least detect when the spectrum is centered. As the spectrum drifts off center, an error signal could be generated to drive the input mirror so that the illumination angle would change and recenter the spectrum. Such a system would have to be capable of following a Doppler drift out to PRF/2 and then rapidly shift back a whole PRF to the next ambiguity spectrum coming into the bandpass of the optical system. The system should have a time constant of several synthetic apertures so that it will be insensitive to terrain changes. Furthermore, to prevent blurring of the output image, the output mirror must be shifted synchronously in the opposing direction to maintain the tracking velocity of the output film drive.

4) Geometric Correction and Location Determination: The conversion of slant range to ground range is not as difficult for spaceborne SAR systems as it is for airborne systems for a given swath width. A much smaller beam covers a given swath width on the ground. In the case of Seasat, the total incidence angle change was about $6^{\circ}$, resulting in a relative ground range scale change of about 28 percent. Breaking the data up into four swaths resulted in an error of about \pm 3.5 percent at each quarter-swath edge when the ground range scale at the center was correct. The use of a nonlinear output film plane or distortion optics could conceivably be used to compensate for the residual error, but loss of resolution would most likely occur.

Doppler centroid drifts from zero Doppler cause a parallelogram-type skew in azimuth proportional to the Doppler angle. A rectangular pattern on the ground will be imaged to a parallelogram where the cross-track sides are not perpendicular to the swath direction. Compensation for such skew can be com- 
pensated for as part of the Doppler tracking system, but the restrictions on mirror positions and angular positional accuracy are severe.

Accurate location is a difficult problem for spaceborne SAR's in general, and there are additional problems in the conventional optical systems. Usually, there are no positional references. Thus relative positions of input data, output recording film, and all optical elements must be precisely maintained. The ability to accommodate image shifts caused by orbital variations, timing changes, and attitude errors is very complex. A very precise and adaptable timing transfer system is required to maintain accurate reference of the input data to a time base during the processes of film recording and correlation to imagery. Some type of reference fiducial must be generated in the imagery, and a computer-generated list of locations of the marks must be made. The experience with Seasat has shown that it is difficult to obtain absolute location-to within a few kilometers-in this type of system.

5) Calibration: There are several potential error sources in an optical processing system which can contribute to calibration problems for a SAR system. In film recording, the diffraction efficiency is highly sensitive to recording spot size, exposure, and development process. All of these parameters must be maintained constant to provide reasonable calibration. Within the correlator itself, spatial and temporal variations of illumination intensity will contribute errors. The development process of the output image film and subsequent copies, again, contributes errors. The use of reference calibration signals can help reduce the errors from the above sources. If a known signal is input into the system, it should undergo the same transfer function as the data, and thus variations due to the processing system could be removed from the output imagery. Unfortunately, it is difficult to make a reference signal with characteristics identical to those of the radar data. Noise can be bandwidth limited in range to simulate the chirp spectrum, but azimuth filtering to simulate the antenna pattern is a complex process. Efforts to calibrate Seasat data using a series of calibrated noise steps resulted in errors of up to $2 \mathrm{~dB}$ contributed by the processor. These errors were in addition to those of the sensor, data link, and data-recording system.

\section{Areas of Current Research and Development in Optical Processing}

As mentioned previously, the primary emphasis in optical processing system research and development has been placed on size, power, and weight reduction. Conventional optical systems have the capability of running at real-time rates, but the use of film as an input and output medium makes the processors large, nonrepeatable, and slow with respect to realtime turnaround. Recent developments of spatial light modulators (SLM's) have supplied an acceptable replacement for film as the input medium to the system. Two basic types of processors are described below. The first type simply uses a two-dimensional SLM as the input, and employs the simple range cylinder with tilted azimuth cylinder correlator described earlier. The second type is quite different, using a one-dimensional acoustic-optic cell input with a charge-coupled device (CCD) array detector at the output.

1) Two-Dimensional, Spatial Light Modulator Correlator: A schematic representation of a two-dimensional correlator using an SLM input is depicted in Fig. 27. The system shown uses a

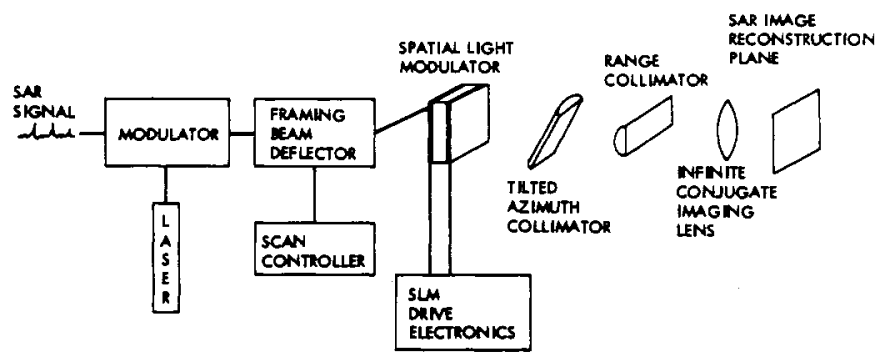

Fig. 27. Schematic of a one-dimensional SAR correlator using spatial light-modulator input.

laser scanning system to write the data in raster format on the SLM. Other types of writing systems could be used such as a CRT or an LED array with a slow mirror scan in azimuth. Such write systems are complex and limit the reduction of system power, weight, and size.

A number of SLM's have been developed by industry, but the prime candidates are the Itek PROM (Pockel's readout optical modulator) and the Hughes LCLV (liquid-crystal light valve). At this point in time, the PROM appears to be more useful because of its higher storage time (tens of seconds as opposed to about $100-\mathrm{ms}$ decay time for the LCLV). The higher storage time is necessary to obtain sufficient synthetic-aperture buildup. A CCD-addressable LCLV is currently under development at Hughes. Such a device would eliminate the need for the input scanning system. A full frame of data is directly inputted into a CCD storage array which is part of the LCLV structure and subsequently strobed onto the liquid-crystal material. It must then be processed before decay occurs. This two-dimensional approach is especially attractive for systems requiring additional data processing such as image spectrum analysis or feature recognition. A coherent two-dimensional image is present at the output, and subsequent processing operations can be easily added.

2) Hybrid Acoustooptic/CCD Correlator: Recent development of two key components have made possible a very small, lightweight, low-power, yet high-performance dataprocessing system. Psaltis [68] at the California Institute of Technology has developed a conceptual design for a twodimensional data processor using laser diode illumination, acoustooptic (A-O) cell data input, and a CCD detector array for the output. Recent development of the laser diodes and CCD array detectors has made the scheme feasible and attractive for various applications. The A-O cell technology has been well developed for some time.

The processing scheme is a hybrid approach in that range correlation is performed spatially as in a conventional system while azimuth correlation is performed temporally as in a time-domain convolution processor. The operation of the system is shown in Fig. 28. A radar echo or PRF line is launched into the A-O cell. The length of cell (in microseconds) corresponds to the length of the range line to be processed. When the echo is fully in the cell, the laser diode is strobed with a pulse short enough to "freeze" the echo. The light is collimated in the range dimension so that range compression occurs through diffraction, much the same way it does in a conventional optical correlator. The range focal plane, which occurs at some distance from the cell, is imaged in range onto the CCD array with the light uniformly dispersed in the vertical or azimuth direction. Each vertical column of the CCD array 


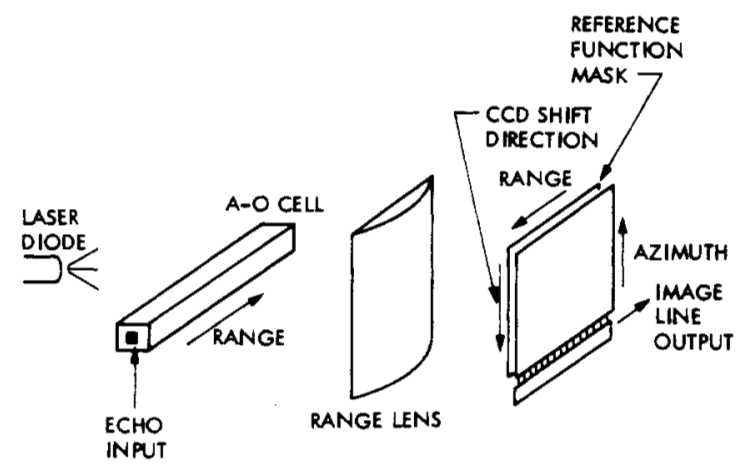

Fig. 28. Schematic of a possible optical SAR processor using a onedimensional acoustooptical input and a CCD array output.

corresponds to a line of constant range (neglecting range curvature). As new range lines come in, rows of data are shifted downward. The transmission mask in front of the CCD array contains the azimuth reference function. The mask and the detector are analogous to a time-domain azimuth correlator. Each CCD detector element, in conjunction with its corresponding value of the reference function, is a multiplier/accumulator. Thus a 300-by-500 array performs 150000 multiplies and adds every PRF line. By the time a particular target shifts from the top to the bottom of the array, it has been fully correlated with the reference function and hence "compressed."

Once the mask is generated (the mask can be computer generated and written on a film recorder), the nominal reference function has been fixed. However, adjustments for "focus" can be made over quite a large range by simply changing the shift rate in the CCD array so that the FM rate of incoming data will match the mask.

Furthermore, Doppler offsets, range walk, and range curvature can be easily accommodated. Since the input video data are placed on a carrier (the center frequency of the A-O cell is usually in the $70-$ to $80-\mathrm{MHz}$ region), the carrier frequency can be offset to null out the Doppler frequency. A given Doppler offset will correspond to a specific range walk. Range walk is caused by targets shifting slightly (in the horizontal direction) as they pass through the antenna beam. This causes the targets to cross range cell columns in the detector. A simple rotation of the array will afford exact tracking of the target trajectories. Although range curvature does not usually occur in the azimuth integration period that current CCD devices can accommodate, it can be easily corrected for by rotated cylindrical lenses in much the same way it was done in the Seasat optical processor.

The above architecture is also highly modular in every dimension. Arrays can be stacked in range for increased swath width. Since the output is complex, they can be stacked in azimuth to provide either higher resolution or more looks. They can also be stacked in operating-bias level to provide increased dynamic range.

Research and development in the detector array field is quite active in industry, and the system performance will improve along with $\mathrm{CCD}$ array improvements. Texas Instruments is expecting to have a 1200 -by-1200 device on the market within the next two years. This device, which is essentially a single chip a few centimeters on a side, would be performing well over $10^{9}$ multiplies/s in the above system and could accommodate $10^{11}$ multiplies/s if required.

\section{Post-Image-Formation Processing}

After the correlation step, the radar data are in a two-dimensional image format, similar to a regular photograph. However, a number of processing steps are still required to achieve radiometric and geometric calibration. The radiometric-calibration step involves corrections for antenna pattern shading, range-tosurface variation, and other known nonlinear processes in the sensor and correlator. The purpose of this step is to have the intensity of each pixel directly proportional (or related by a known function) to the surface backscatter cross section. The geometric calibration step involves adjustment of the spatial location of each pixel in the image such that it could be superimposed on a well-defined cartographic grid.

A number of other post-image-formation processing techniques have also been used as a first step to facilitate information extraction. Some of these techniques are reviewed in this section. They are texture analysis, speckle statistics, registration to visible and IR images, color coding, and stereo imaging. Some of these techniques are still at an early stage of development; however, they seem to have good potential.

\section{A. Geometric Calibration}

The SAR uses the time delay and the Doppler (or phase) history to locate a particular target on the surface relative to the satellite orbit. These two measurements are not dependent on the satellite attitude, which is the case with passive sensors. For the SAR, the determination of pixel location is mainly dependent on the accuracy of knowledge of the spacecraft location, on the internal timing of the signals, and on the characterization of the processing algorithm. A detailed analysis of the effects of the different error sources was given by Curlander and Brown [25] and Curlander [23]. Based on the analysis of Seasat SAR data over a number of sites, they concluded that pixels can be located with an absolute accuracy better than $200 \mathrm{~m}$ (i.e., 8 resolution elements). This is definitely not a basic limit. With a more favorable illumination geometry (larger incidence angle) and tighter requirements on the ephemeris accuracy, absolute accuracy down to about a few tens of meters or a few resolution elements is definitely feasible.

\section{B. Radiometric Calibration}

Radiometric calibration involves correction to the image such that the surface backscatter cross section can be derived directly from the image pixels' intensity. Depending on the application, different levels of calibration are desired. In some cases, only relative calibration of pixels in a scene or relative calibration between images taken at different times is needed. In other cases absolute calibration is necessary.

Errors due to the sensor elements can be corrected by: 1) continuously monitoring the level of the transmitted signal and the transfer function of the receiver; 2) measuring the antenna radiation pattern before launch and making ground receiver measurements during the flight; and 3) measuring the transfer function of the ground processor. Preliminary analysis conducted using the Seasat SAR data indicates that rms relative temporal calibration between different passes over the same area is $\pm 0.5 \mathrm{~dB}$ [Held and Croft, private communication]; however, more research in this area is still required.

Another factor which plays an important role in the SAR calibration is the presence of speckle. The coherent noise will add uncertainty to the measured intensity of a specific pixel as 

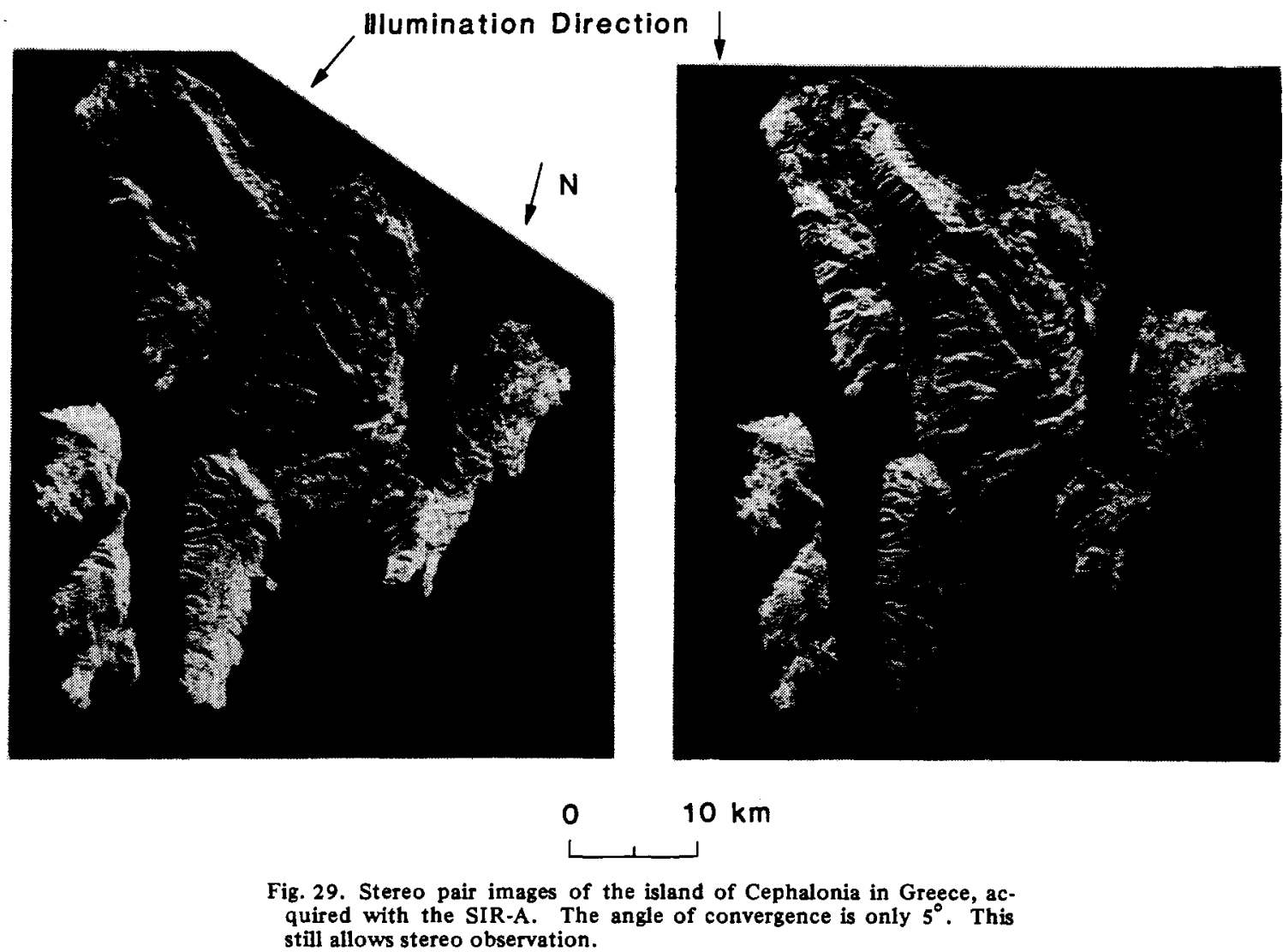

discussed earlier. In order to minimize the ratio of the standard deviation to the mean intensity, a large number of looks must be acquired. This, in turn, will degrade the resolution. Thus a tradeoff must be made between spatial resolution and "radiometric resolution." This has been discussed by Moore $[61]$.

\section{Texture Analysis}

One of the features which is used in the analysis of an image is its texture. The texture is defined as the spatial variation in the image brightness on the scale of a few tens of resolution elements.

A number of researchers have developed and used analytical techniques to study and classify texture in radar images. Shanmugan et al. [75] and Blom and Daily [10] used spectral analysis to classify geologic units based on their texture in the radar image. Blom and Daily [10] used a processing technique of splitspectrum to enhance the identification of geologic structures in radar images. In this technique they generated a false-color image where the hue is proportional to the lowfrequency spatial components of the image spectrum and the intensity is proportional to the high-frequency spatial components.

\section{Speckle Statistical Properties}

The speckle in the radar image is a result of the interaction of the coherent electromagnetic wave with a rough surface. It is basically the interference pattern resulting from the combination of the retums from the different scattering points on the surface. Thus the statistical properties of the speckle as a function of the observing frequency are dependent on the surface roughness.
In his paper, Jain [46] used the decorrelation properties of the speckle as a function of the radar frequency to derive the Ims height of ocean waves imaged with the SAR. If the wave height is small, a slight change in the observing frequency will induce only slight change in the speckle pattern, i.e., high correlation. If the wave height is large, a slight change in the observing frequency will completely change the speckle pattern, implying strong decorrelation. This technique is of particular interest because it will extend the capability of SAR use in observing ocean-surface wave patterns.

\section{E. Multisensors Registration}

The radar provides an image of the surface scattering properties in the microwave region. Thus the combination of radar images with visible/IR images will provide us with a more complete picture of the surface scattering and emission properties over most of the electromagnetic spectrum which is accessible to spaceborne remote sensors.

The digital registration of radar data (airborne and spaceborne) to Landsat data was reported by Daily et al. [27] and Blom and Daily [10]. This required a good deal of effort for a number of reasons: 1) the data were taken from different platforms, and 2) the two types of sensors have different imaging geometry (i.e., radar uses a range-time format, while Landsat uses an angle-angle format in the image formation). The foreshortening in the radar images makes it particularly complicated to register accurately images of surfaces with extensive topographic change.

The analysis of the combined Seasat-Landsat images provided encouraging results. Blom and Daily [10] analyzed the combined images of the San Rafael Swell region in eastern Utah. They found that the addition of the Seasat data im- 


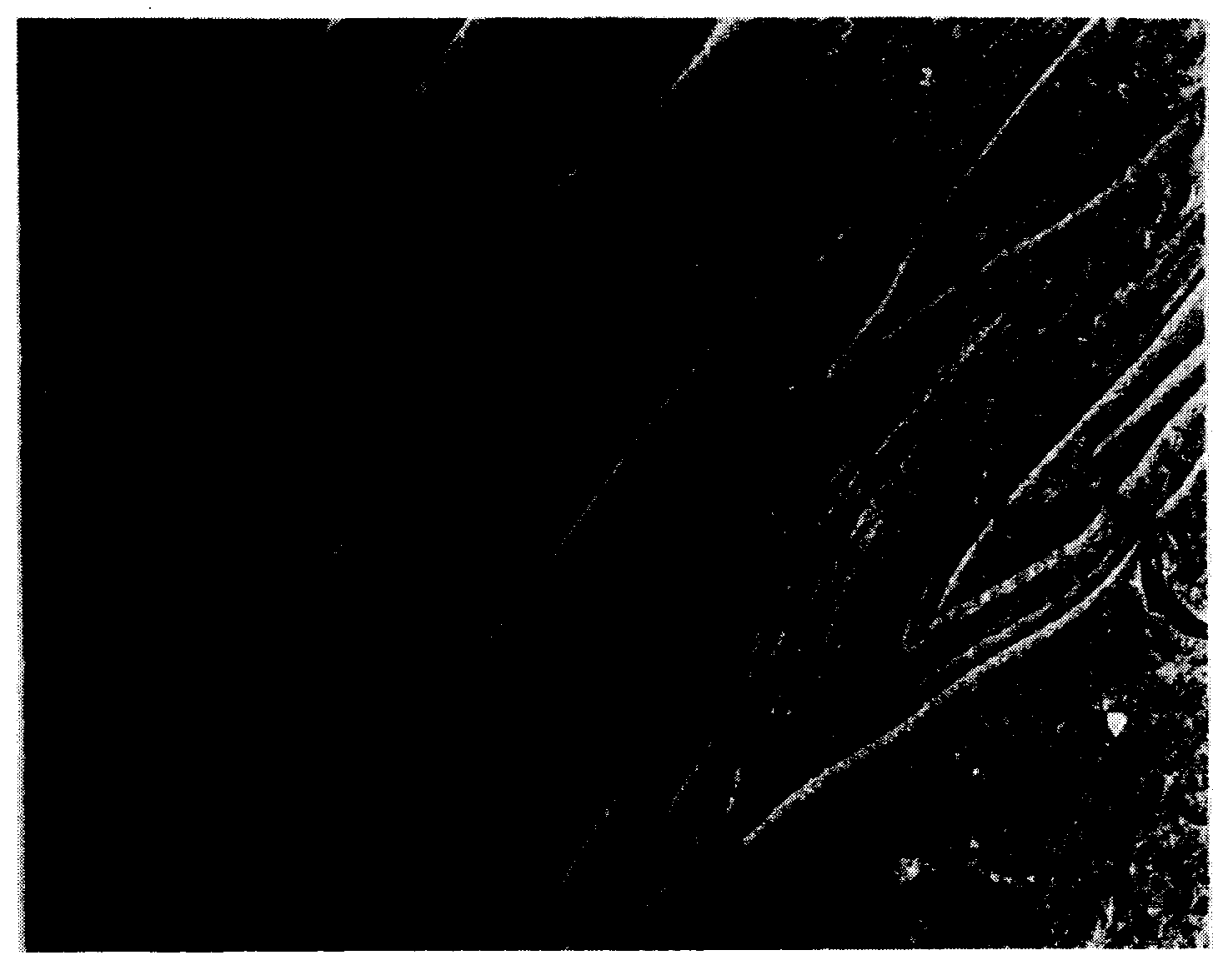

(a)

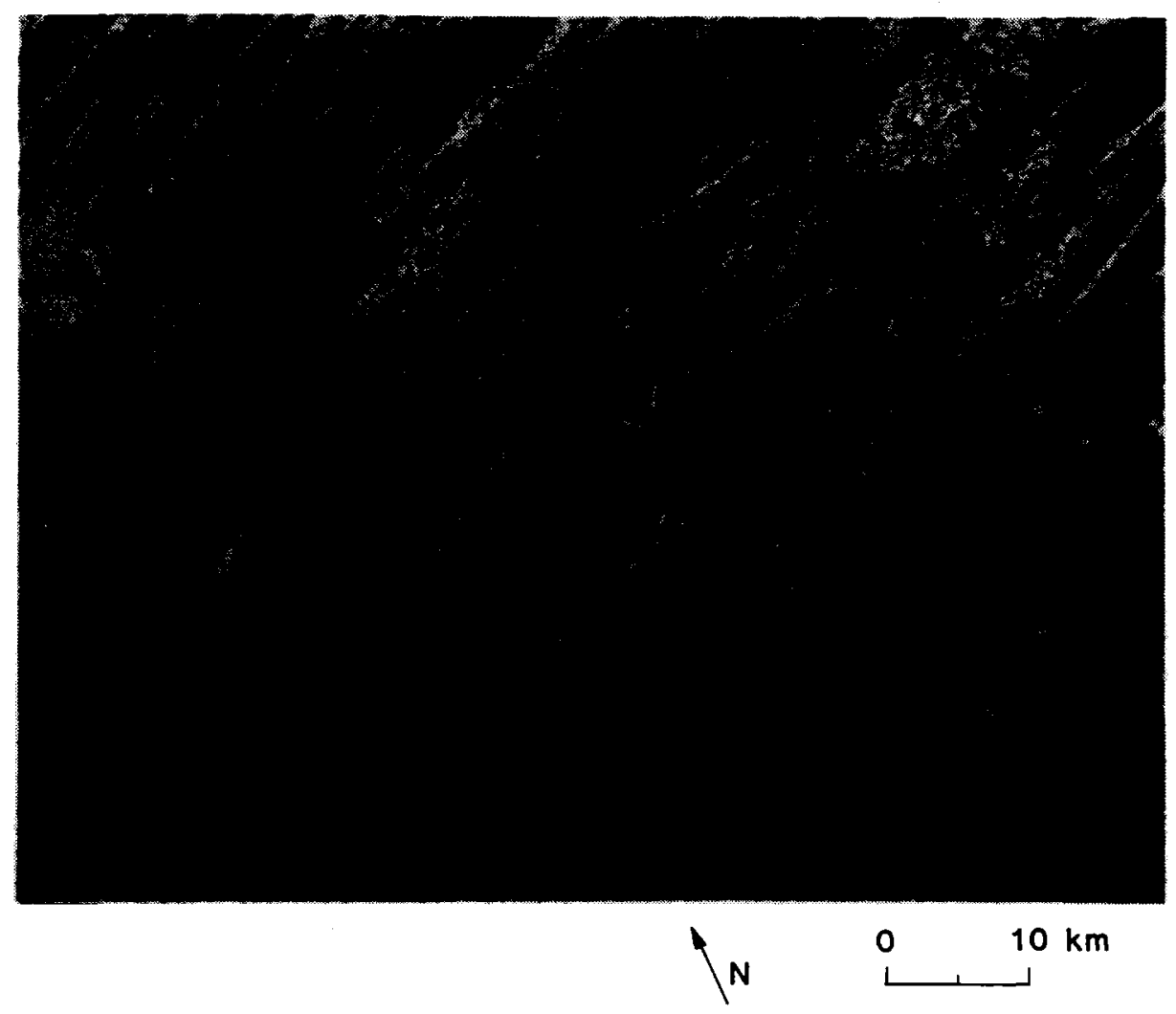

(b)

Fig. 30. Seasat (a) and Landsat (b) images of the Appalachians near Harrisburg, PA. The Susquehanna River is visible in the upper right corner. The pattern of "noses" formed by the mountains and valleys represents plunging structures formed by the folded layers. 


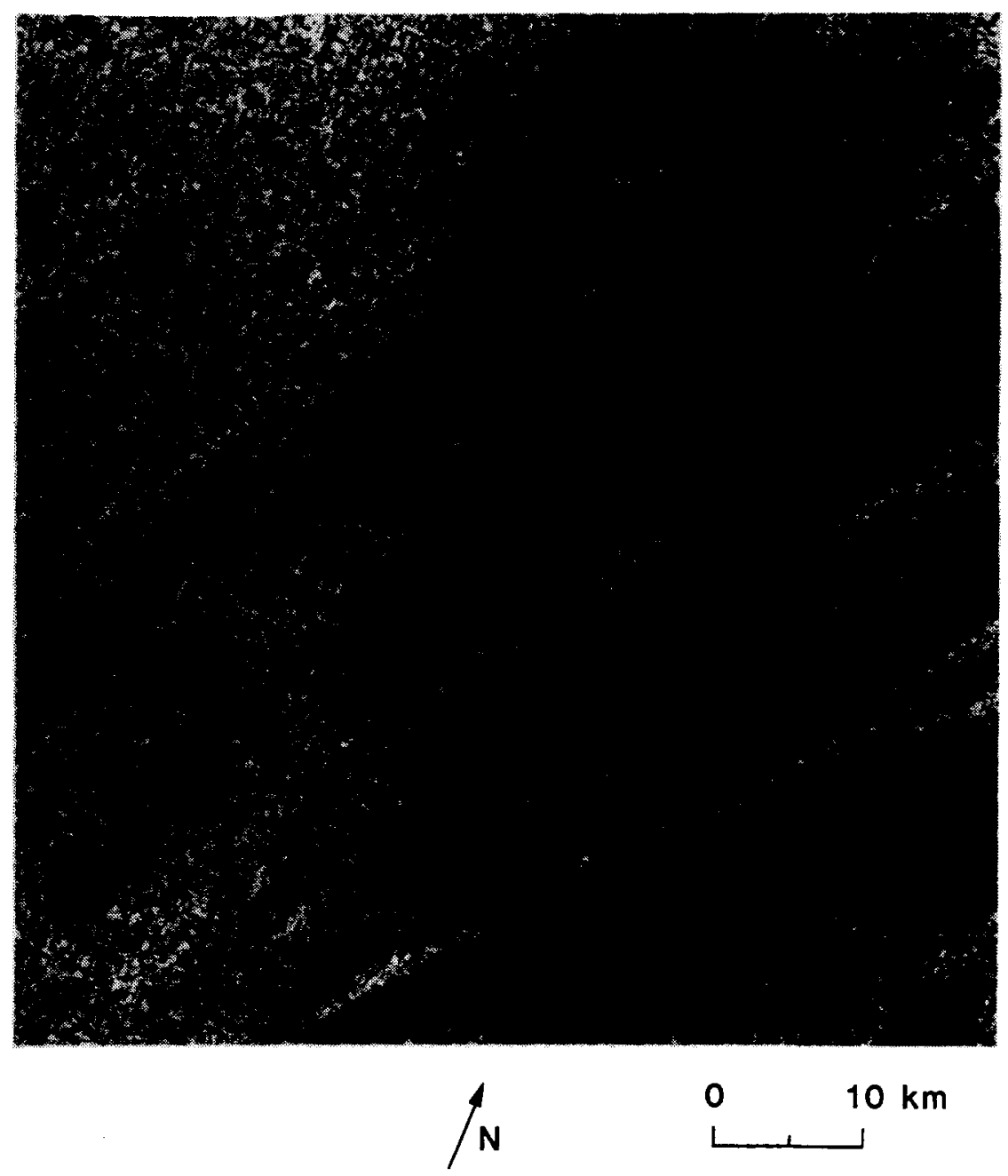

Fig. 31. Seasat image of cultivation fields around Ames, IA.

proved the lithographic classification capability from 59 percent with Landsat alone to 72 percent with the combined data set.

\section{F. Stereo Imaging}

The radar sensor can provide stereo images in a manner similar to visible sensors. In this case, two orbital passes are required such that two images of the same area can be acquired with two incidence angles.

In the case of Seasat and SIR-A, only limited stereo capability was possible. Because the look angle was fixed, small convergence-angle stereo was acquired in limited cases when a certain area was observed in the far edge of the swath on one orbital pass and in the near edge of the swath on another orbit. Fig. 29 shows such a pair of images.

\section{DATA INTERPRETATION TECHNIQUES AND APPLICATIONS}

The tone of the radar image is a representation of the surface backscatter cross section, which in turn is a function of the surface slope, its roughness at the scale of the radar wavelength, and its complex dielectric constant. For relatively long radar wavelengths, the volumetric properties of the near-surface region also play a role in the backscatter return.

The interpretation of the SAR images and raw data is based on three types of information: 1) geometric patterns, forms, and shapes; 2) image tone and texture; and 3) coherency properties, such as speckle statistics and Doppler shift. Examples of the first one are lineaments, folds, dunes, drainage patterns, cultivation fields boundaries, ocean-wave patterns, current boundaries, ice flows, etc. These patterns, forms, and shapes are interpreted in a way similar to that used with regular photography. Fig. 30 shows a Seasat SAR and a Landsat image of the folded Appalachians near Harrisburg, PA. The folded structures, a series of anticlinal and synclinal features, are clearly recognizable on both images for the same reason, i.e., a recognizable variation of image tone in an organized pattern which, from field experience, is usually associated with folding of geologic strata.

Image tone and texture are primarily a function of the surface roughness and subresolution small-scale topography, the surface complex dielectric constant, and surface variations on the scale of few resolution elements. The dielectric constant variation is most useful in the study of vegetated and moist surfaces. The tonal and textural data on the radar image provide new information that is not available with optical or IR photography. Interpretation of the data requires an understanding of the interaction of microwaves with natural surfaces [74], [11]. Fig. 31 shows a Seasat SAR image of cultivation fields in Central Iowa. The tonal variations in the SAR image are not directly correlated with the tonal variations observed on Landsat images. This shows that the surface properties 

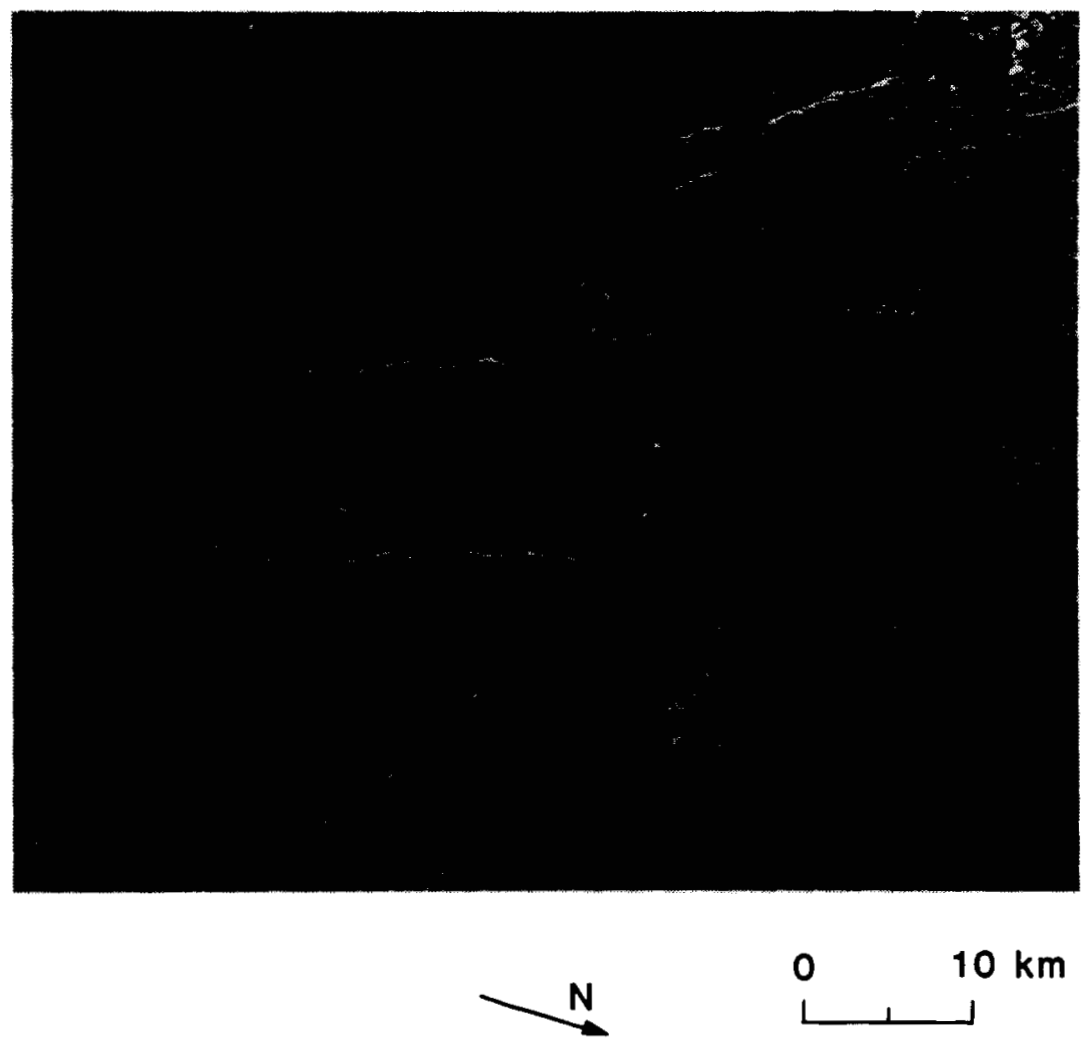

Fig. 32. Seasat image of Central Jamaica. The Blue Mountains in eastern Jamaica represent a pre-Eocene igneous and metamorphic complex. They show as coarse texture. Younger limestones cover most of the rest of the island and form karst topography which shows as fine denture.

have different effects on the response of each sensor. Work is presently ongoing, mostly at the University of Kansas and JSC, on how to use these two types of data in a complementary way to improve the discrimination and identification of crops.

Fig. 32 is a Seasat SAR image of Jamaica. The area in the image is completely covered by vegetation. Variations in the image texture are clearly visible. These variations correlate well with the surface geology [28]. The fine texture corresponds to limestone areas where the karst topography is well developed. The coarse texture corresponds to areas with igneous or metamorphic rocks. The topographic texture is visible on the radar image because of the strong sensitivity of the backscatter intensity to variations in the surface slope.

The third type of information is unique to coherent radar sensors. The surface roughness and texture of the subresolution scale leads to interference patterns which are represented by the speckle in the radar image. The statistical properties of the speckle are related to the surface geometric properties, and recent research has been conducted on deriving surface height statistics, particularly for ocean waves [46], using the speckle decorrelation properties as a function of observation frequency.

The target motion (for example, ocean currents and waves, vessels, man-made objects, etc.) also has an effect on the Doppler shift of the echo. Research is ongoing to measure the surface motion magnitude and spatial distribution from the radar data.

The Seasat SAR data, and the recently acquired SIR-A data, are being used in a number of applications related to the Earth and Ocean Sciences. In this section, we present a brief overview of the main research results in the fields of geology, oceanography, glaciology, and agriculture with some discussion about future potentials of the SAR sensor.

\section{A. Geologic Mapping Applications}

In geologic mapping applications, the SAR images are used to detect, delineate, map, and identify features, patterns, shapes, albedo variations, and texture variations, and their relative spatial relationship. The image brightness, which is a direct representation of the intensity of the radar backscatter, is mainly an effect of the surface slope, roughness (surficial and volumetric), and dielectric constant.

On a qualitative basis, radar images are interpreted in a way similar to that used with regular photography [60], [5], [72] . The radar sensor has the advantage that the angle of illumination, and to some extent the direction of illumination, are selectable. This is not the case with spaceborne visible and IR sensors where the geometry is completely fixed by the position of the Sun and the time of data acquisition. Proper illumination geometry is of particular importance for structural mapping. Thus the radar and visible IR sensors will complement each other by providing different spectral signatures and illumination geometry.

Quantitative analysis of the radar imaging albedo is somewhat more involved. Models have been developed, and to some extent tested, to relate surface roughness and dielectric constant to the radar return. However, there is still a need for appreciably more work to be able to interpret quantitatively the albedo in radar images.

Fig. 33 is a SAR image of the central part of the Dominican Republic. Fig. 34 shows simplified interpretation maps of the 

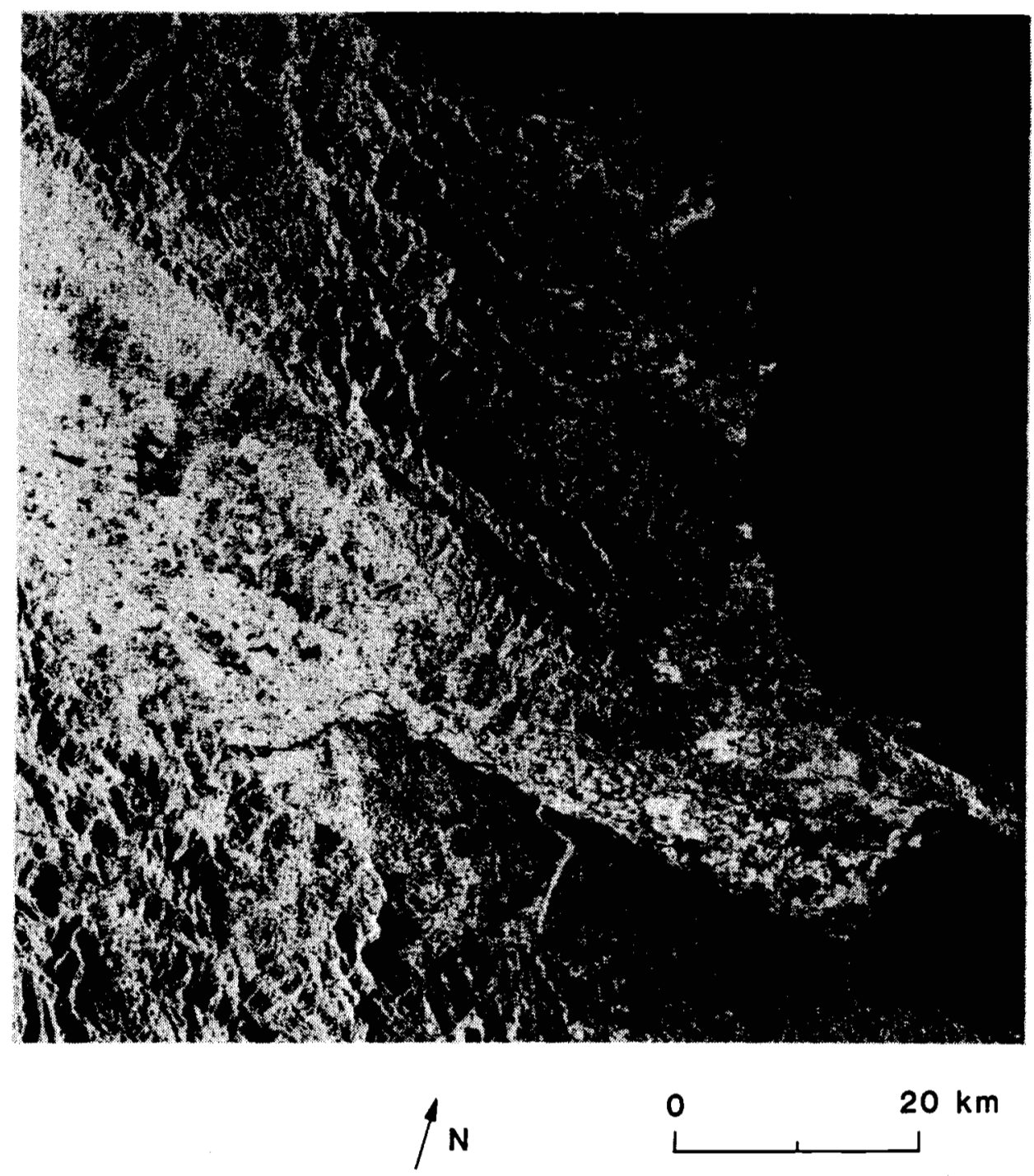

Fig. 33. Seasat image of the northeast part of the Dominican republic. Three major topographic units are visible. The youngest deposits of flat-lying soils and grounds occupy the central region. Tertiary limestones that give rise to karst topography (fine texture) cover the lower right region in the image. Cretaceous and older volcanic rocks underlie the more rugged mountainous basement in the rest of the image.

lineaments and the texture in the radar image. Lineaments are visible as an abrupt, spatially linear, change in image brightness [73], in texture (see Fig. 33), in drainage-flow direction (see Fig. 33), or topography (see Fig. 32). Linear features mapped on a radar image must be verified on the ground to determine their exact nature. Large-scale linear features are usually associated with faulting. Variation in texture is usually associated with change in lithology. In Figs. 32 and 33, the fine texture corresponds to Tertiary limestone units which formed karst topography. The very coarse texture corresponds to igneous and metamorphic units. The rest of the area corresponds to Quaternary alluvium.

In a detailed comparative analysis of the Seasat SAR and Landsat images of the Pine Mountain thrust region near Knoxville, TN, Ford [37] concluded that these two sensors are complementary for lineament mapping. The Seasat SAR seems to be superior because of the sensitivity of radar backscatter to surface slope change, and because it allows at least two different illumination directions. However, the use of both sensors does allow a more complete mapping capability.
The spatial relationship of surface features is an important geologic indicator. Fig. 35(a) shows the radar image of Central Ireland. This is a region covered with drumlins (elongated, well-defined hills) which are remnants of the glacial period. The alignment of these hills reflects the retreat pattern of the ice sheet ([38]; see Fig. 35(b)).

Fig. 36 shows a simpler and more direct case of identification based on spatial relationship. A very bright region is seen emanating from the bottom of a cone-shaped feature with a circular crater at the top. This occurs in a volcanic region north of Flagstaff, AZ. This can be immediately interpreted as a very rough, recent lava flow emanating from a well-defined cinder cone. A detailed analysis of radar images of volcanic fields is given by Schaber $e$ t al. [74].

More subtle features could be enhanced by special processing. Blom and Daily [10] used color enhancement of slight albedo and texture variations to detect and delineate a geologic structure in the Patrick Draw, WY. The geologic feature, a subsurface arch, was almost invisible on the surface. However, the surface texture, which is mainly controlled by the drainage 


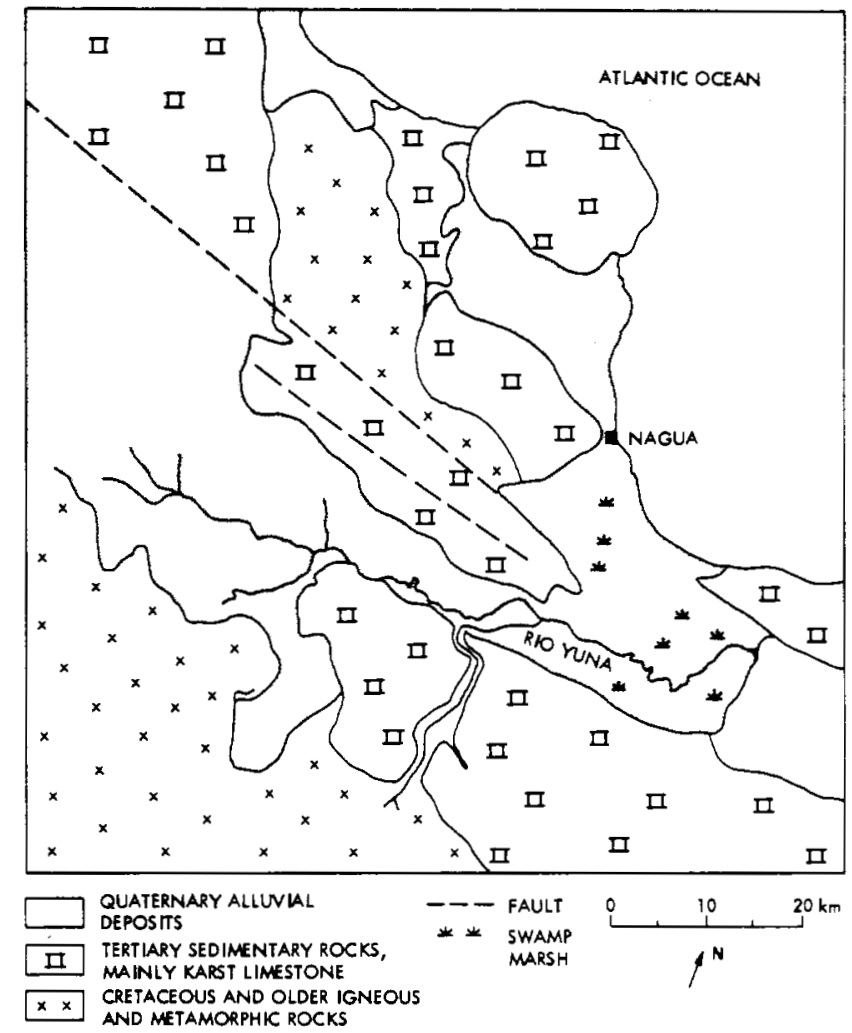

Fig. 34. Simplified map of lineaments and texture observed in Fig. 33. (Courtesy of T. Dixon.)

patterns, and the surface albedo, which is mainly affected by the vegetation cover, were slightly affected by the regional subsurface structure. Blom and Daily [10] used a digital combination of Seasat and Landsat images of the San Rafael Swell region to enhance the capability of classifying the lithologic units in that region.

Another type of geologic feature, sand dune fields, was studied by Blom and Elachi [11]. In this case, the surface consists of a number of smooth tilted facets, and the scattering occurs primarily in the specular mode; that is, strong echoes are returned from the dunes' facets that are properly oriented (e.g., normal or near normal) relative to the radar illumination.

The effect of the illumination geometry is illustrated in Fig. 37, which shows the Seasat and SIR-A images of the Santa Ynez Mountains. Because of the near-vertical illumination of the Seasat SAR, the foldover effect leads to extensive distortions in the mountainous area, making interpretation very difficult. In the case of the SIR-A data, the large incidenceangle illumination allows a much better view of the layered structure in the mountainous terrain. However, in the flat areas, the Seasat image seems to contain additional useful information on the surface drainage. Thus it seems that a dual(or multiple-) incidence angle illumination will be required to acquire a full picture of the surface structure.

\section{B. Oceanographic Applications}

In oceanographic applications, the imaging radar sensor has a unique and essential characteristic-the capability to acquire high-resolution images of the surface regardless of cloud cover and at any time of the day or night. This characteristic is essential because of the dynamic nature of almost all the features on the ocean surface. A review of spaceborne imaging radar applications in oceanography is given by Beal et al. [6] .

The radar sensor provides an image that is representative of the surface backscatter characteristics. In the case of the ocean, the backscatter is completely controlled by the smallscale surface topography: the short gravity and capillary waves which scatter the radar energy by the Bragg scattering mechanism, and the local tilt of the surface, which is due to the presence of large waves and swells. Thus the SAR is capable of imaging surface and near-surface phenomena that affect the surface roughness directly or indirectly. These phenomena include surface waves, internal waves, currents, weather fronts, wind or oil slicks, and eddies. In this section, we discuss examples of ocean features that have been observed with Seasat SAR. Some of these observations have not been verified by simultaneous ground truth; however, we will present the most logical interpretation. The Seasat SAR provided for the first time a synoptic high-resolution view of large ocean areas; and in some cases, it provided repetitive observations of the same region every three days. Some of the observed features were never observed before, and their interpretation is controversial at the present time. Other features were observed to occur much more commonly than had previously been thought.

Surface waves are visible on the radar image as a periodic regular change in the image tone (Fig. 38 ). The spatially periodic change in the surface-coherent backscatter cross section is a result of three surface effects that are modulated by the presence of a propagating surface wave or swell: 1) local slope; 2) the intensity and bunching of small gravity and capillary waves; and 3) the wave orbital velocity, which affects the phase of the returned echo. The relative importance of these three effects is not yet well understood [36], [29], [3], [71], [2] .

Fig. 38 shows a Seasat image of ocean surface waves acquired over the northeastern Atlantic, near the Shetland Islands. The surface waves had a wavelength of about $300 \mathrm{~m}$, and the refraction patterns near the coast are clearly visible. This Seasat image was acquired in $14 \mathrm{~s}$. Thus it represents an almost in- 

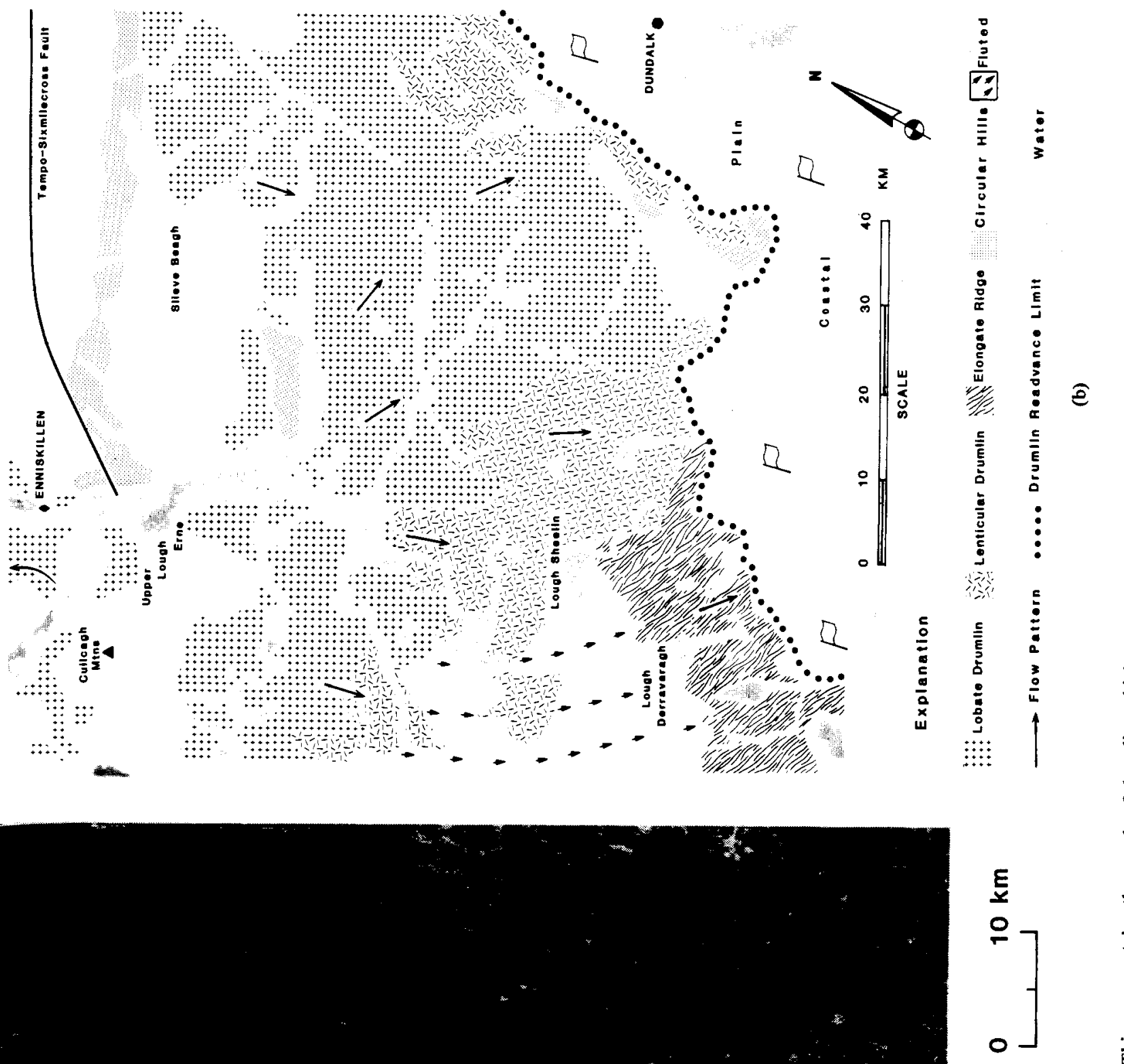


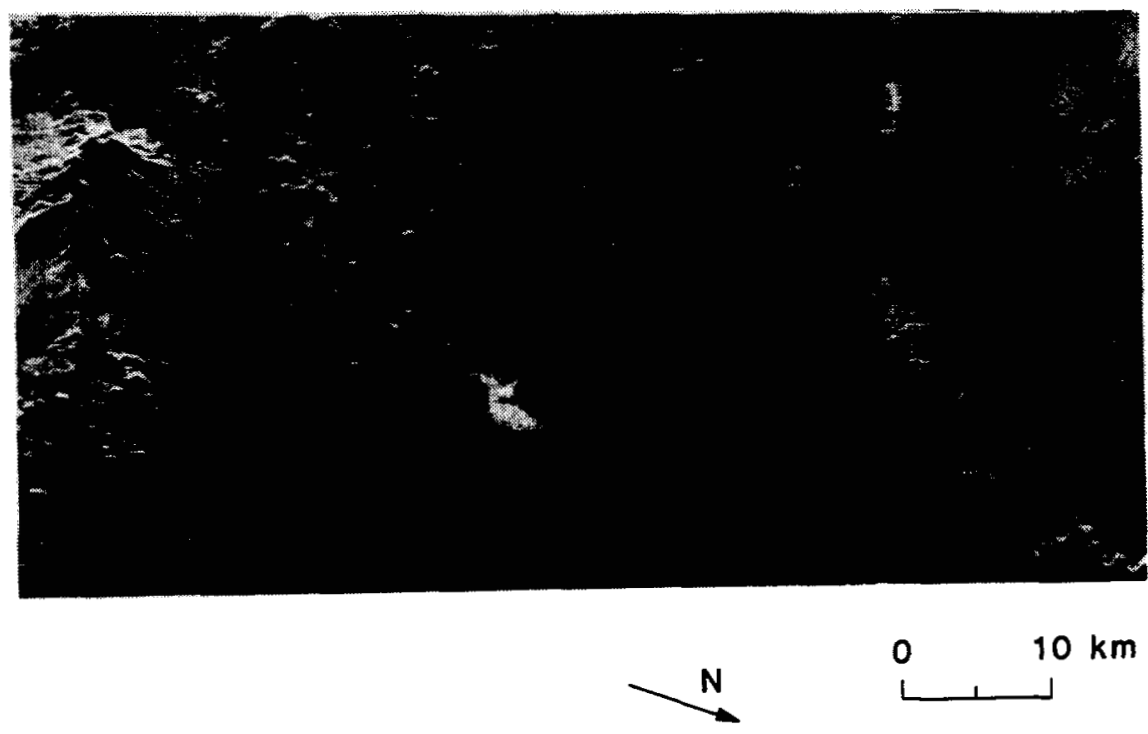

Fig. 36. Seasat image of the San Francisco volcanic field, north of Flagstaff, AZ. Numerous cinder cones and craters are visible. The brightest area corresponds to the lava flow emanating from the SP cinder cone.

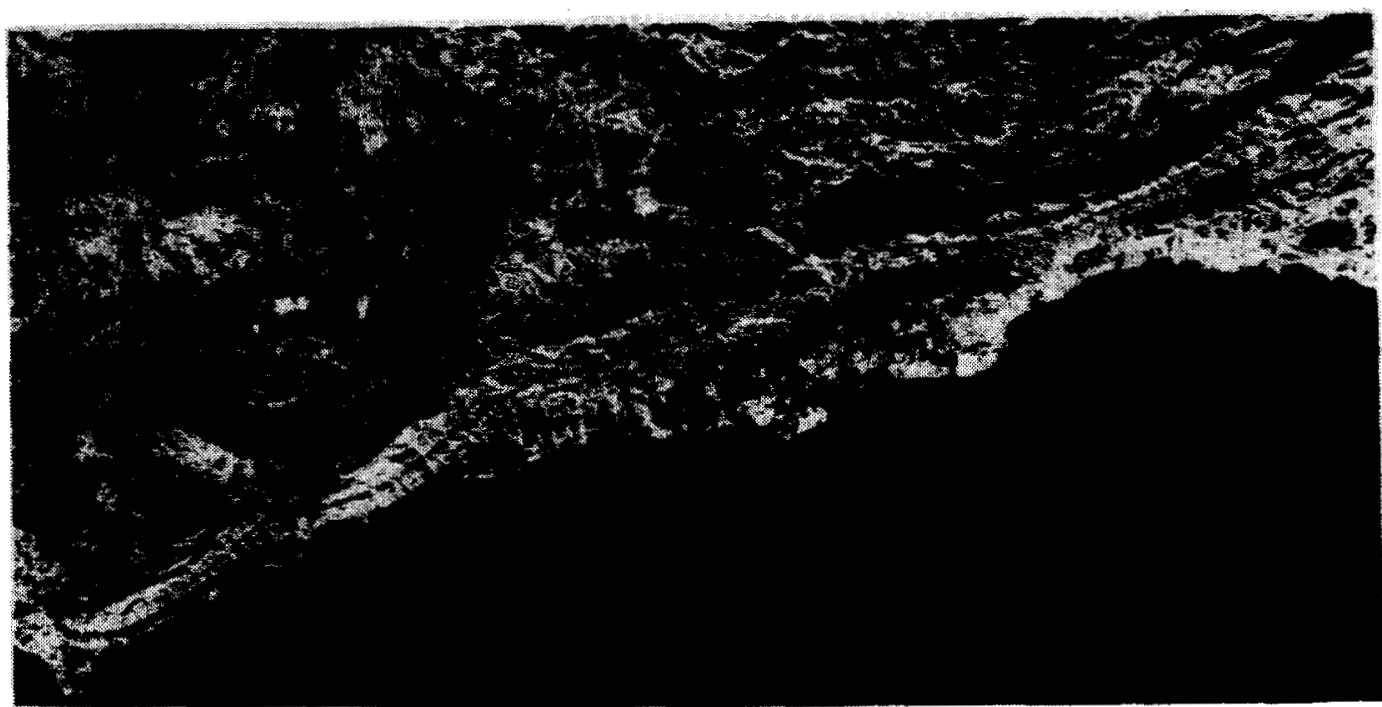

(a)

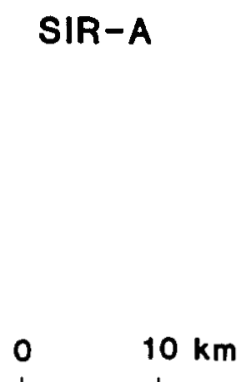

$10 \mathrm{~km}$

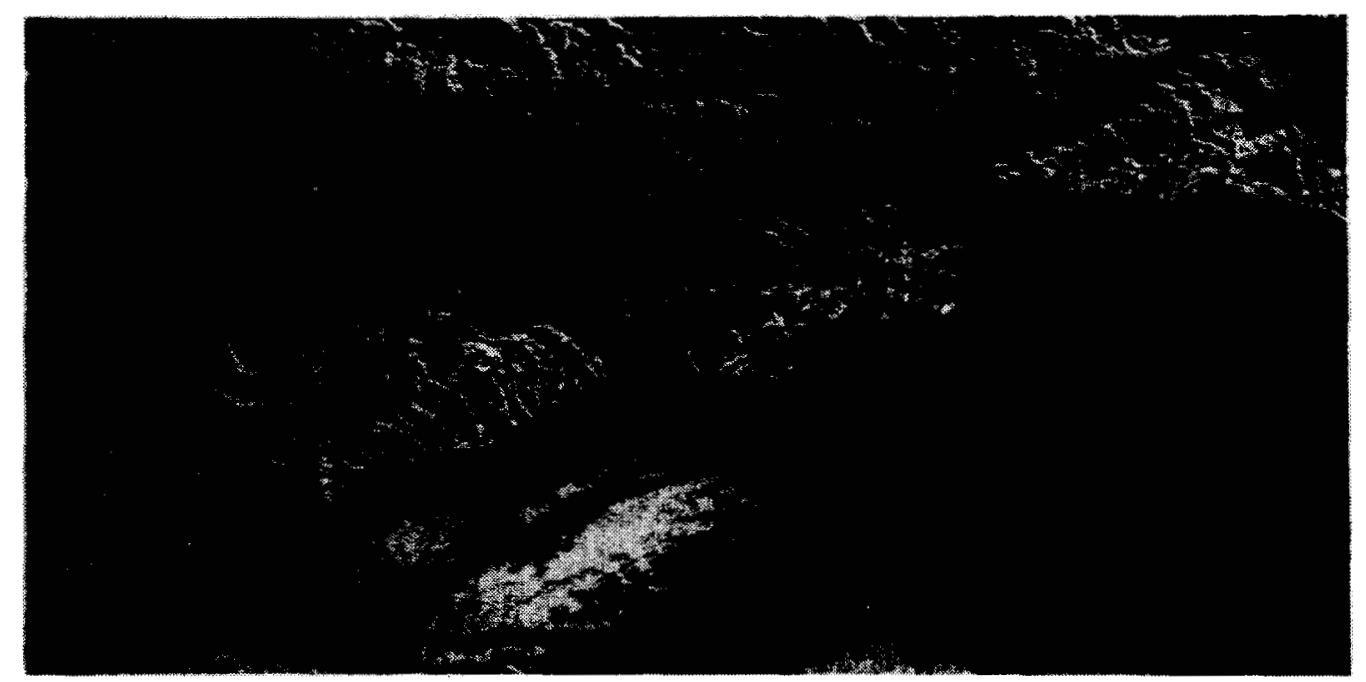

(b)

Fig. 37. Seasat (a) and SIR-A (b) images of the Santa Ynez Mountains along the California coastline near Santa Barbara. The SIR-A image is clearly superior to study the structure in the mountainous region. The Seasat image provides additional information in relatively flat regions. 

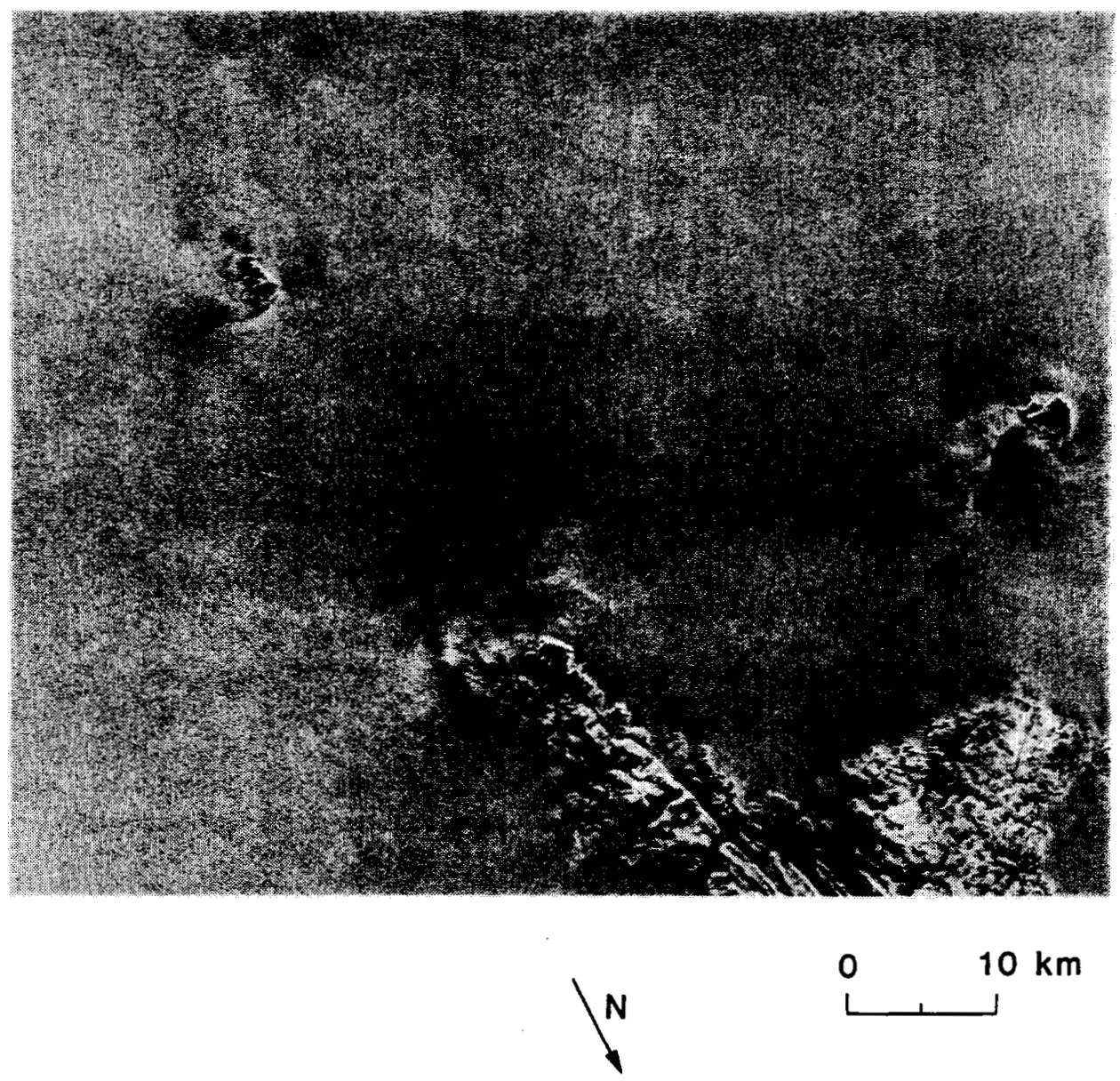

Fig. 38. Seasat image of surface ocean waves near Shetland Island, England. The swell had a wavelength of $300 \mathrm{~m}$. The two small isiands are Foule and Fair Isle. Observe the refraction and diffraction of the swell near the coast.
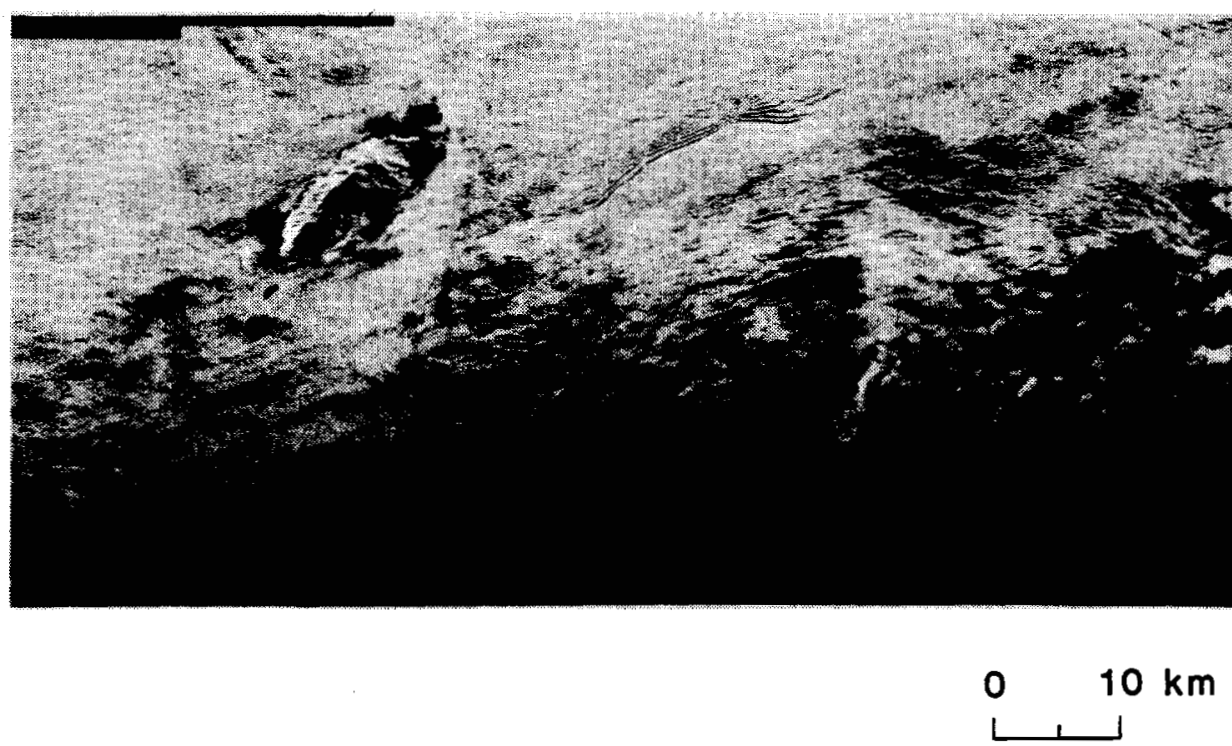

Fig. 39. Seasat image of internal waves near the island of San Nicolas off the coast of California.

stantaneous snapshot of the wave pattern over this $100 \times 100$ $\mathrm{km}$ region.

Fig. 39 shows internal waves in the western Atlantic just east of Manhattan Island. These waves are observed as a result of their surface manifestations and their effect on the surface roughness. The rather large currents associated with these waves modify the capillary-ultragravity surface-wave spectrum overlying the oscillations. The exact mechanisms by which 

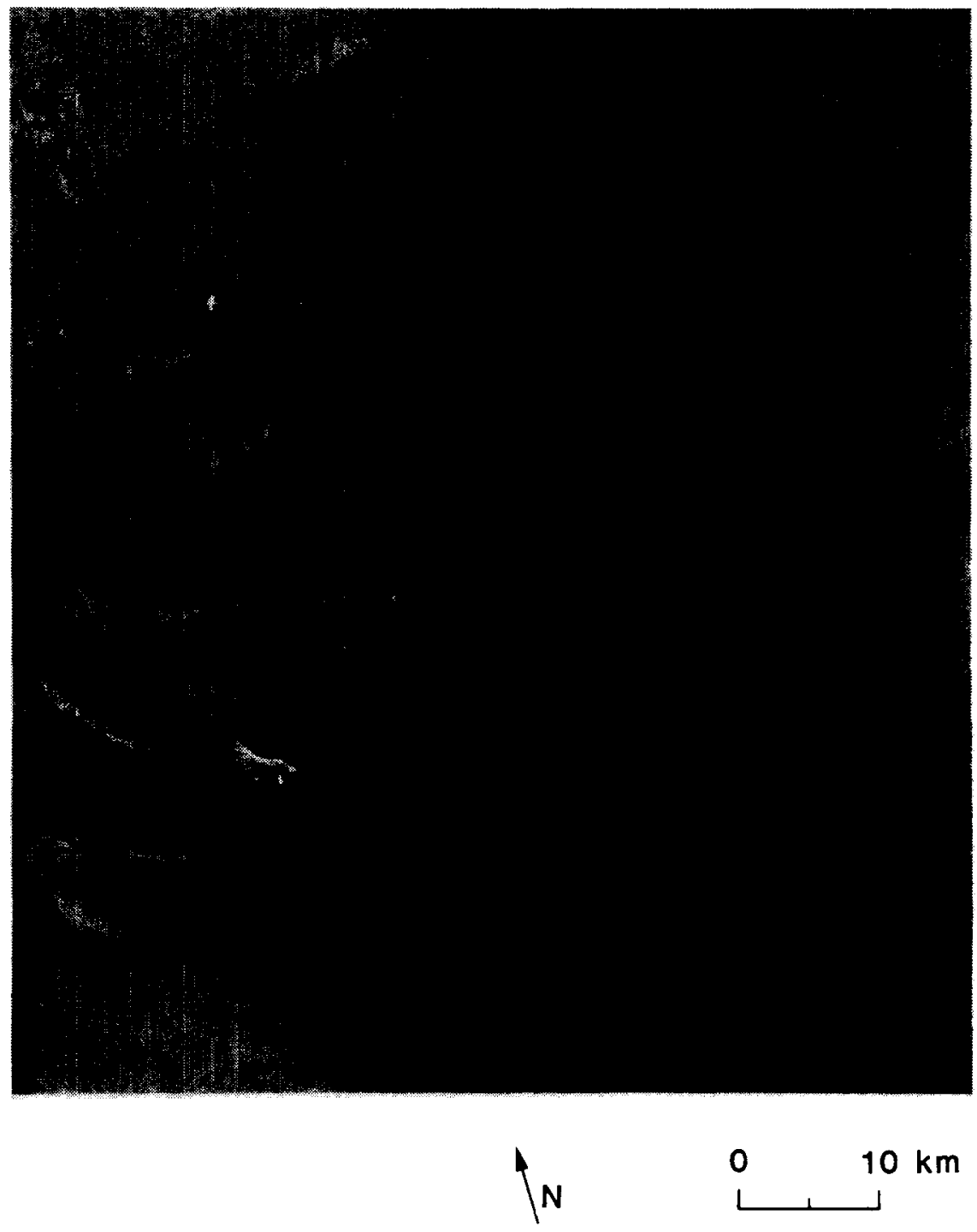

Fig. 40. Seasat image of the Nantucket Island region. The island is visible in the upper left corner of the image. Most of the other patterns observed on the ocean surface reflect the bottom topography in this shallow region.

the modifications take place are still the subject of discussion, but at least two hypotheses have been advanced [40]. According to the first hypothesis, the high velocity of surface water arising from the internal wave amplitude can sweep surface oils and materials together to form a smooth strip near regions of surface water convergence. The second mechanism predicts that capillary and ultragravity wave energy is concentrated in the convergence zone by surface-current stress, which then becomes a region of enhanced roughness rather than a smooth area as with the first hypothesis. When such smooth and rough regions are illuminated away from normal incidence and then viewed at nonspecular angles, the smooth region would appear darker and the rough one brighter than the normal sea surface. This geometry is the same for both imaging radar and multispectral (including optical) sensors.

Internal waves are usually observed on the radar image as a wave packet that consists of a series of convex strips, with the spatial periodicity becoming shorter toward the center of curvature. The length of the crest may range up to many tens of kifometers. The leading wavelengths are on the order of 1 to $2 \mathrm{~km}$ and decrease monotonically toward the rear. They usually occur in groups or packets, and they have been observed in numerous places along the western and eastern coasts of North America. Similar observations have been conducted with aircraft SAR [34] and optical sensors [4]. On some Seasat single swaths, more internal waves could be observed than the total number observed during dozens of aircraft flights over a period of five years. This illustrates the new insight that resulted from the Seasat SAR experiment on the extent and rate of occurrence of certain dynamic ocean phenomena.

An interesting phenomenon which was observed for the first time with the Seasat radar is shown in Fig. 40. The different patterns observed in the shallow waters around Nantucket Island correlate very closely with the bottom bathymetry. The radar waves do not penetrate the ocean surface sufficiently to sense the bottom topography. The most likely interpretation is that the change in the thickness of the water column modulates the velocity of the near-surface current. This in turn modulates the ocean surface roughness which is the main physical parameter that the radar is sensing. Thus indirectly, the radar image reflects the bottom topography. 


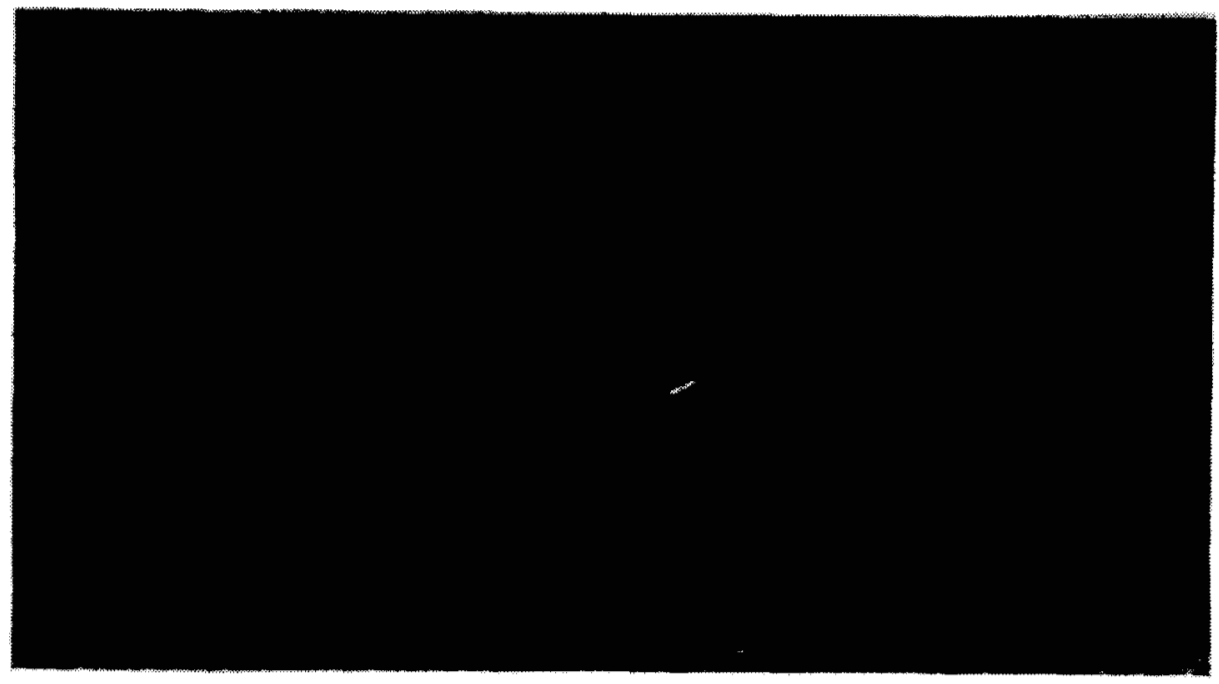

October 1,1978

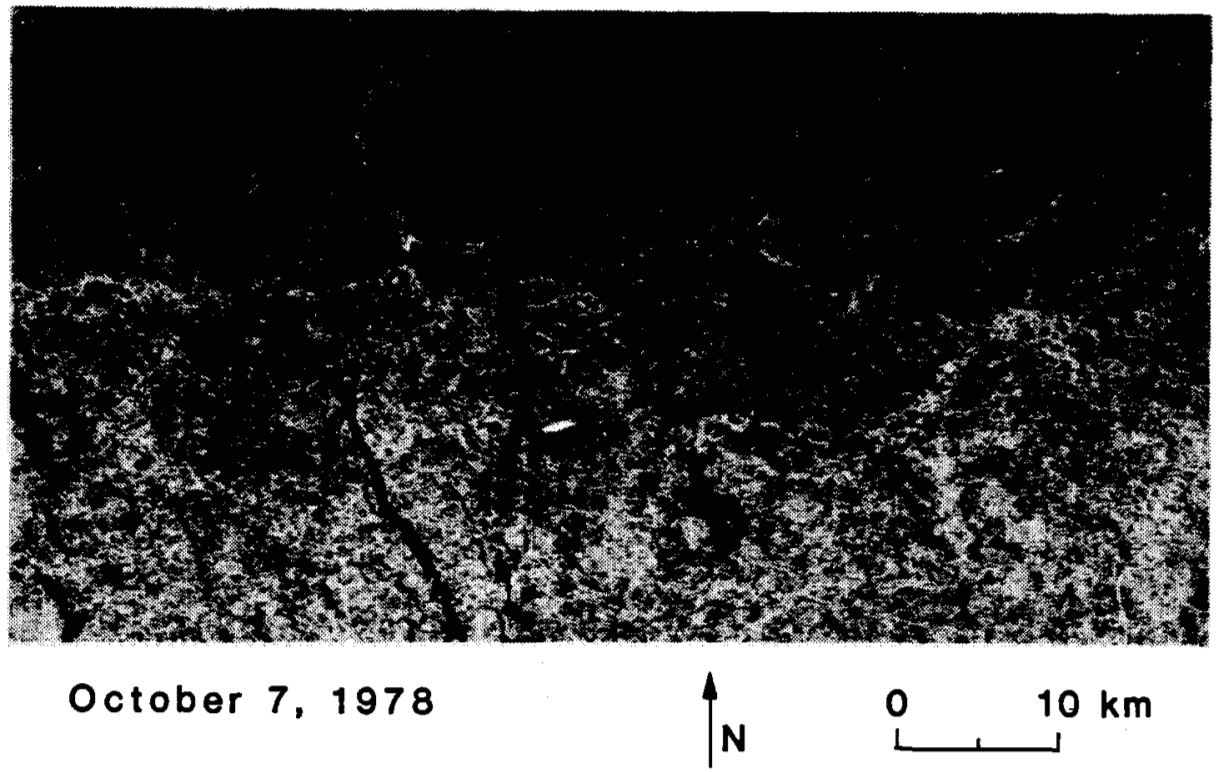

Fig. 41. Seasat image of polar ice floes in the Beaufort Sea acquired six days apart. The bright section is most likely a stranded iceberg.

Other features which were observed on radar images include current boundaries, eddies, and vessels.

\section{Polar Ice and Glaciology Applications}

Spaceborne imaging of the polar ice cover is of particular interest in two areas: 1) mapping of the ice-cover motion and extent and spatial distribution of the open water channels, and 2) determination of the ice floes' age.

A spaceborne imaging SAR is the ideal sensor for mapping the ice spatial distribution, extent, and motion. The all-time capability of the radar allows repetitive observation all through the year. Global coverage every three to four days can be easily achieved at the polar regions. For this application, the main observable characteristics are the shape, size, and distribution (including accurate spatial location) of the ice features [55]. Leberl et al. [54] have shown that the polar ice motion can be mapped with the Seasat data to an accuracy of $150 \mathrm{~m}$ or better and that furture systems should be able to achieve a location accuracy of better than $50 \mathrm{~m}$. Fig. 41 shows an example of a Seasat image of an ice region which was observed over a period of about two months in the summer of 1978. Fig. 42 shows the motion of that region during that time period.

To be able to determine the ice type and age, a better understanding of the wave-surface interaction mechanisms is required. At the present time, it seems that a multiparameter radar system might be necessary in order to classify uniquely which ice floes are recently formed, one year old, or many years old.

Fig. 43 is the Seasat SAR image of the Malaspina Glacier in southeastern Alaska. The ice-floe patterns are observed because of the folded moraines, which usually have different roughness characteristics from the surrounding ice.

\section{Renewable Resources}

Landsat data are being used to observe, identify, estimate the extent of, and monitor the growth of different crops on a regional scale. The use of the surface cover reflection in the visible and near-IR spectral regions has been very successful; however, it is limited by the clouds which, over many world regions, could last for many weeks at a time. Thus a combina- 


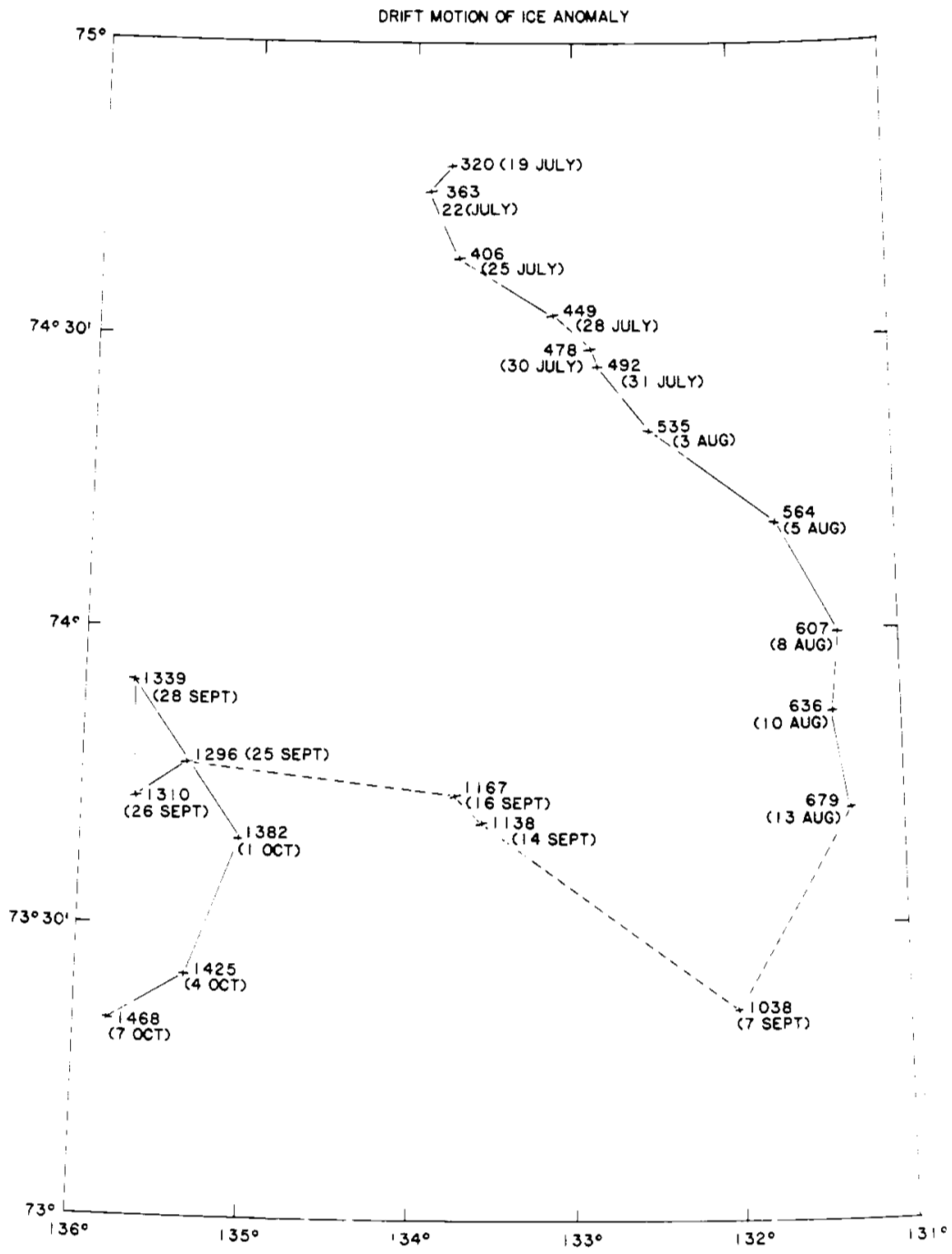

Fig. 42. Long-term motion of the bright feature in Fig. 41. (Courtesy of B. Holt.)
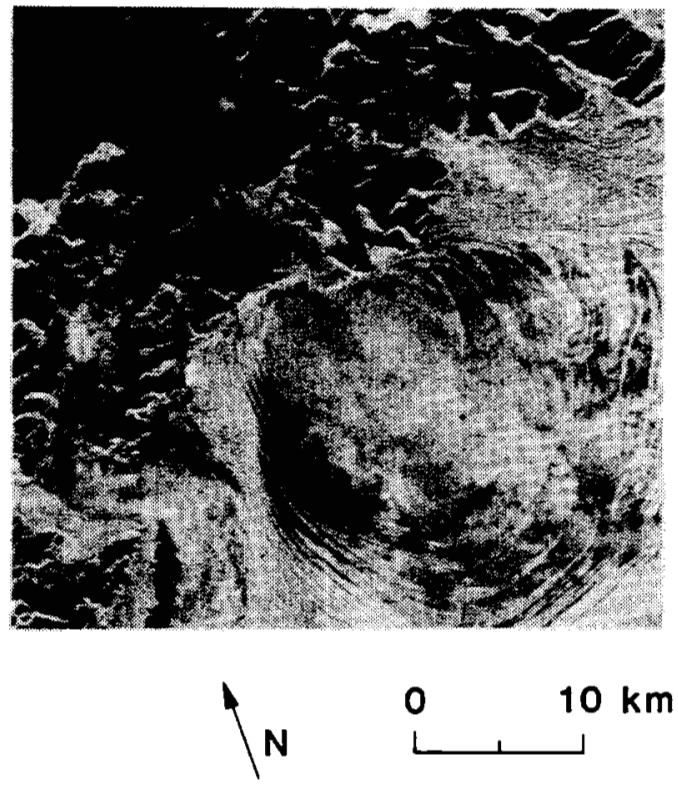

Fig. 43. Seasat image of the Malispina glacier, AK. The ice-floe pattern is visible as a series of bright and dark curvilinear regions. These correspond to the areas of different roughness. tion of visible/IR and microwave imaging sensors will allow a continuous monitoring capability where the radar sensor will be used mainly to observe changes during the cloudy periods, and the visible/IR sensor will provide identifications at the preand post-cloudiness times.

Fig. 31 is a Seasat SAR image over Ames, IA. The distribution of the cultivation fields is clearly visible. Different fields have different albedo; however, it is not yet well understood how the radar albedo relates to the nature and properties of the surface and the surface cover. An interesting effect is observed on this image-an increase of the albedo over the upper half of the image and over streak-like areas in the lower half. The most likely interpretation is that this increase in the average albedo is due to an increase in the soil moisture as a result of a rainstorm which occurred just a few hours before the data were acquired. This same effect was observed in numerous other places where post-rain images showed bright albedo streaks while pre-rain images of the same areas did not.

\section{The Next Decade}

The Seasat SAR demonstrated that high-resolution synoptic radar images can be acquired from space and can be used to continuously monitor the Earth's surface. The analysis of the 
jeasat SAR data clearly showed that spaceborne radar sensors Ire essential for monitoring ocean-surface dynamic phenomena ind for improved structural mapping, particularly in tropical ind arid regions. They also play a complementary role to Jandsat-type sensors for lithologic mapping and crop monitorng. Thus the imaging radar sensor is an important element of he spaceborne sensors that will be used in the 1980's and seyond for Earth observation.

The success of the Seasat SAR experiment and of the SIR-A las led to an international interest in developing and flying nore sophisticated spaceborne SAR's. In 1983 an $X$-band 3AR, will be flown by the Germans on Spacelab. For the mid 1980's, the U.S. is planning multiparameter Shuttle radar sys:ems which have a wide range of flexibility and will be used nainly for research purposes. The SIR-B (1984 flight) will rave a controllable incidence angle. The SAMEX (Shuttle Active Microwave Experiment, 1987-1990 flights), will operate it $L$ - and $C$-band at all polarizations, and with a variable incilence angle. The data handling and processing will be completely digital. This sensor could also be operated from an orbiting platform for long-duration missions.

For long-term observations, a number of free-flying SAR systems are under study by the U.S., Japan, Canada, and the ESA. These systems will have less flexibility; however, their main challenge is in the fast turnaround of the data. They all will require a real-time digital correlator system, which is a major technological development.

In the area of data analysis, a number of research topics will be of particular interest during the next decade. These include the use of speckle statistics to classify surface unit, use of the Doppler information to map ocean currents, use of multispectral multipolarization radars for lithologic and surface cover classification, and the use of multi-incidence angle imaging for structural mapping and stero imaging.

Finally, a mission to map the cloud-hidden surface of Venus is planned for the late 1980's. This mission, called Venus Radar Mapper, will use an orbiting synthetic-aperture imaging radar very similar to the ones used by Seasat and SIR-A. Later, in the 1990's, there are plans to image the surface of Titan with an orbiting radar.

\section{ACKNOWLEDGMENT}

The work reported in this paper would not have been possible without the help of many of our colleagues who are involved in the radar remote sensing program at JPL. They are too many to list here, but they all contributed in some way or other to this work. We would also like to acknowledge the excellent support of Ms. Olivia Tyler and Ms. Sue Conrow in preparing the artwork and typing the manuscript.

\section{REFERENCES}

[1] J. Aarons, H. E. Whitney, and R. S. Allen, "Global morphology of ionospheric scintillations," Proc. IEEE, vol. 59, pp. 159-172, 1971.

[2] W. R. Alpers, D. B. Ross, and C. L. Rufenach, "On the detectability of ocean surface waves by real and synthetic aperture radars," J. Geophys Res, vol. 86, pp. 6481-6498, 1981.

[3] W. R. Alpers and C. L. Rufenach, "The effect of orbital motion on synthetic aperture imagery of ocean waves," IEEE Trans. Antennas Propagat., vol. AP-27, pp. 635-690, 1979.

[4] J. P. Apel, H. M. Byrne, J. R. Proni, and R. L. Charnell, "Observations of oceanic internal and surface waves from the Earth Resources Technology Satellite," J. Geophys Res, vol. 80, pp. 865-881, 1975 .

[5] T. E. Avery, Interpretation of Aerial Photographs, 3rd. ed. Minneapolis, MN : Burgess, 1977

[6] R. C. Beal, P. S. Deleonibus, and I. Katz, Spaceborne Synthetic
A perture Radar for Oceanography. Baltimore, MD: John Hopkins Univ. Press, 1981.

[7] J. R. Bennett, I. G. Cumming, and R. A. Deane, "The digital processing of SEASAT synthetic radar data," in Proc. IEEE Int. Radar Conf., pp. 168-175, Apr. 1980.

[8] J. R. Bennett and P. R. Mc Connell, "Considerations in the design of optimal multilook processors for image quality," presented the SAR Image-Quality Workshop, paper ESA SP-1 72, Frascati, Italy, December 11, 1980.

[9] M. Benson, "Digital processing of SEASAT-A SAR data using linear approximations to the range cell migration curves," in Proc. IEEE Int. Radar Conf., pp. 176-181, Apr. 1980.

[10] R. Blom and M. Daily, "Radar image processing for rock type discrimination," IEEE Trans, Geosci Remote Sensing, vol. GE-20, pp. 343-351, July 1982.

[11] R. Blom and C. Elachi, "Spaceborne and airborne imaging radar observation of sand dunes," J. Geophys Res, vol. 86, pp. 3061-3070, 1981.

[12] G. Born, J. A. Dunne, and D. B. Lowe, "SEASAT mission overview," Science, vol. 204, pp. 1405-1406, 1979.

[13] E. Brookner, "Present and future trends in radar signal processing," Trends and Perspectives in Signal Processing, vol.I, no. 4, Oct. 1981 .

[14] W. E. Brown, Jr., C. Elachi, and T. W. Thompson, "Radar imaging of ocean surface patterns," J. Geophys Res, vol. 31, pp. $2657-2667,1976$.

[15] W. M. Brown, G. G. Houser, and R. G. Jenkins, "Synthetic aperture processing with limited storage and presuming," IEEE Trans A erosp. Electron. Syst, vol. AES -9 , pp. 166-176, 1973.

[16] W. M. Brown and L. J, Porcello, "An introduction to syntheticaperture radar," IEEE Spectrum, vol. 6, pp. 52-62, 1969.

[17] A. A. Burns and E. J. Fremouw, "A real-time correction technique for transionospheric ranging error," IEEE Trans Antennas Propagat., vol. AP-1 8, pp. 785-790, 1970.

[18] J. P. Claassen and J. Eckerman, "A system concept for wide swath constant incidence angle coverage," in Proc. Synthetic Aperture Radar Technology Conf. (New Mexico State Univ., Mar. 8-10, 1978).

[19] IEEE Computer (Special Issue on Array Processors), vol. 15, Sept. 1981.

[20] C. Cook and M. Bernfeld, Radar Signals: An Introduction to Theory and Application. New York: Academic Press, 1967, sec. 6.3 and 7.3 .

[21] R. K. Crane, "Ionospheric scintillation," Proc. IEEE, vol. 65, pp. 180-199, 1977.

[22] I. G. Cumming and J. R. Bennett, "Digital processing of Seasat SAR data," in Proc. IEEE Int. Conf. Acoustic, Speech, Signal Process (Washington, DC, Apr. 1979), pp. 710-717.

[23] J. C. Curlander, "Location of spaceborne SAR imagery," IEEE Trans. Geosci Remote Sensing, vol. GE-20, pp. 359-364, July 1982.

[24] J. Curlander, F. Li, and C. Wu, "Estimate of Doppler parameters for spaceborne synthetic aperture radar processing," IEEE Trans A erosp. Electron. Syst., submitted for publication, 1982.

[25] J. C. Curlander and W. E. Brown, "A pixel location algorithm for spaceborne SAR imagery," presented at the IGARSS Conf. , Washington, DC, June 8, 1980.

[26] L. J. Cutrona, "Synthetic aperture radar," in Radar Handbook, M. I. Skolnik, Ed. New York: McGraw-Hill, 1970.

[27] M. Daily, C. Elachi, T. Fart, R. Stromberg, S. Williams, and G. Schaber, "Application of multispectral radar and landsat imagery to geologic mapping in Death Valley, California," Jet Propulsion Lab., Pasadena, CA, Tech. Rep. 78-14, 1978.

[28] T. Dixon, private communication, 1982.

[29] C. Elachi, "Radar imaging of the ocean surface," Boundary Layer Meteoroh, vol. 13, pp. 154-173, 1977.

[30] _- "Shuttle imaging radar: Research sensor for earth resources observation," presented at the IGARSS Conf., Washington, DC, June 1981.

[31] —-, "Spaceborne imaging radar: Geologic and oceanographic applications," Science, vol. 209, pp. 1073-1082, 1980.

[32] - "Wave patterns across the North Atlantic on September 28, 1974 from airborne radar imagery," J. Geophys. Res, vol. 81, pp. 2655-2656, May 20, 1976.

[33] C. Elachi et aL, "The shuttle imaging radar (SIR-A): Preliminary results," Science, submitted for publication, 1982.

[34] C. Elachi and J. Apel, "Internal wave observations made with an airborne synthetic aperture imaging radar," Geophys. Res. Lett., vol. 3 , pp. $647-650,1976$.

[35] C. Elachi, R. Goldstein, and D. Held, "Spaceborne bistatic SAR," presented at the IGARSS Conf., Washington, DC, 1981.

[36] C. Elachi and W. E. Brown, "Models of radar imaging of the ocean surface waves," IEEE Trans Antennas Propagat., vol. AP-25, pp. 84-95, Jan. 1977; also IEEE J. Oceanic Eng., vol. OE-2, pp. 84-95, Jan. 1977.

[37] J. Ford, "Seasat orbital radar imagery for geologic mapping: Tennessee-Kentucky-Virginia," Amer. A ssoc. Pet. Geologists, 
vol. 66, p. 2064, 1980.

[38 ] - "A contrast in landform mapping from Seasat radar images," Quaternary Geology, to be published, 1982.

[39] J. Ford, R. Blom, M. L. Bryan, M. Daily, T. Dixon, C. Elachi, and $H$. Xenos, Seasat Radar Views America, the Caribbean and Western Europe, Jet Propulsion Lab., Pasadena, CA, publication 80-67, 1980 .

[40] H. E. Garrett and B. A. Hughes, "On the interaction of surface and internal waves," J. Fluid Mech., vol. 52, pp. 179-191, 1972.

[41] J. W. Goodman, Introduction to Fourrier Optics. New York: McGraw-Hill, 1968.

[42] J. Granger, "Shuttle imaging radar-A," presented at the IGA RSS Conf., Washington, DC, June 1981.

[43] J. P. Guignard, "Overview of digital processing of SAR data," in Proc. 15th Int. Symp. on Remote Sensing of Environment (Ann Arbor, MI, May 1981).

[44] R. O. Harger, Synthetic Aperture Radar Systems: Theory and Design New York: Academic Press, 1970.

[45] D. Held and N. Mehta, "Calibration of synthetic aperture radars: The effects of nonlinearities," presented at the IGARSS Conf., Washington, DC, June 1981.

[46] A. Jain, "Determination of ocean wave heights from synthetic aperture radar imagery," Appl. Phys, vol. 13, pp. 371-382, 1977.

[47] F. Janza, "Interaction mechanisms," in Manual of Remote Sensing, R. G. Reeves Ed. Falls Church, VA: Amer. Soc. of Photogrammetry, 1975, ch. 4.

[48] R. L. Jordan, "The Seasat-A synthetic aperture radar system," IEEE J. Oceanic Eng., vol. OE-5, pp. 154-163, 1980.

[49] R. L. Jordan and B. L. Huneycutt, "Seasat-A synthetic aperture radar performance," presented at the Int. Conf. Communications, Boston, MA, ICC'79, June 10-13, 1979.

[50] J. C. Kirk, "A discussion of digital processing for synthetic aperture radar," IEEE Trans. Aerosp. Electron Syst., vol. AES11, pp. 326-337, May 1975.

[51] A. Kozma, E. M. Leith, and N. G. Massey, "Tilted plane optical processor," A ppl Opt., vol. 11, pp. 1766-1777, 1972.

[52] J. J. Kovaly, Synthetic A perture Radar. Dedham, MA: Artech House, 1976.

[53] T. R. Larson, L. I. Moskowitz, and J. W. Wright, "A note on SAR imagery of the ocean," IEEE Trans Antennas Propagat., vol. AP-24, pp. 393-394, May 1976.

[54] F. Leberl, J. Raggam, C. Elachi, and W. Campbell, "Sea ice motion measurements from Seasat SAR images," $J$. Geophys. Res, submitted for publication, 1982.

[55] F. Leberl, M. L. Bryan, C. Elachi, T. Farr, and W. Campbell, "Mapping of sea ice measurement of its drift using aircraft synthetic aperture radar images," J. Geophys Res., vol. 84, pp. 1827-1835, Apr. 20, 1979.

[56] E. N. Leith, "Complex spatial filters for image deconvolution," Proc. IEEE, vol. 65, pp. 18-28, 1977.

[57] -, "Quasi-holographic techniques in the microwave region," Proc. IEEE, vol. 59, pp. 1305-1318, Sept. 1971.

[58] F. Li and H. Zebker, "A digital Seasat SAR correlation-simulation program," in Dig. 1981 Geoscience and Remote Sensing Symp., Vol. II, IEEE Geoscience and Remote Sensing Society, pp. 548-554.

[59] L. Martinson, "A programmable digital processor for airborne radar," in IEEE 1975 Int. Radar Conf. Rec., pp. 186-191, Apr. 1975 .

[60] V. C. Miller, Photogeology. New York: McGraw-Hill, 1961.

[61] R. K. Moore, "Tradeoff between picture element dimensions and noncoherent averaging in side-locking airborne radar," IEEE Trans Aerosp. and Electron. Syst., vol. AES-15, pp. 697-708, 1979.

[62] R. K. Moore, J. P. Claassen, and Y. H. Lin, "Scanning spaceborne synthetic-aperture radar with integrated radiometer," IEEE Trans. Aerosp. Electron. Syst., vol. AES-17, pp. 410$419,1981$.

[63] R. E. Morden and F. Powell, "SAPHIRE design and development," Goodyear Aerospace Corporation, Arizona Division, Litchfield Park, AZ, paper GERA-2 177, Rev. A, Sept. 1977.
[64] N. Nohmi, N. Ito, and S. Hanaki, "Digital processing of spaceborne SAR data," European Space Agency Rep. SP-172, Dec. 1980.

[65] M. Ono et aL, "Digital processing of SEASAT SAR data using CRAY-1," European Space Agency, Rep. ESA SP-172, (SAR Image Quality), pp. 45-46.

[66] W. J. Peeples, W. R. Sill, T. W. May, S. H. Ward, R. J. Phillips, R. L. Jordan, E. A. Abott, and T. J. Killpack, "Orbital radar evidence for lunar subsurface layering in mare serenitatis and crisium," J. Geophys Res, vol. 83, pp. 3459-3468, 1978.

[67] L. J. Porcello, R. Jordan, J. S. Zelenka, G. F. Adams, R. J. Phillips, W. E. Brown, S. H. Ward, and P. Jackson, "The Apollo lunar sounder radar system," Proc. IEEE, vol. 62, pp. 769-783, 1974.

[68] D. Psaltis, private communication, 1981

[69] R. K. Raney, "Synthetic aperture imaging radar and moving targets," IEEE Trans. Aerosp. and Electron. Syst., vol. AES-7, no. 3, pp. 499-505, May 1971.

[70] A. W. Rihaczek, Principles of High-Resolution Radar. New York: McGraw-Hill, 1969.

[71] C. L. Rufenach and W. R. Alpers, "Imaging ocean waves by SAR with long integration times," IEEE Trans Antennas Propagat., vol. AP-29, pp. 422-428, 1981.

[72] F. F. Sabins, Remote Sensing: Principles and Interpretation. San Francisco: Freeman, 1978.

[73] F. F. Sabins, R. Blom, and E. Elachi, "Seasat radar image of the San Andreas Fault, California," Amer. Assoc. Pet. Geol., vol. 64, p. 614, 1980 .

[74] G. G. Schaber, C. Elachi, and T. Farr, "Remote sensing data of SP lava flow and vicinity in North Central Arizona," Remote Sensing of the Environment, vol. 9, p. 169, 1980.

[75] K. S. Shanmugan, V. Narayanan, V. S. Frost, J. A. Stiles, and J. C. Holtzman, "Textural features for radar image analysis," IEEE Trans. Geosci. Remote Sensing, vol. GE-19, pp. 153-156, 1981.

[76] K. Tomiyasu, "Conceptual performance of a satellite borne, wide swath SAR," IEEE Trans. Geosci. Remote Sensing, vol. GE-19, pp. 108-116, 1981.

[77] —, "Tutorial review of synthetic aperture radar with application to imaging of the ocean surface," Proc IEEE, vol. 66, pp. $563-583,1978$.

[78] T. K. Truong, I. S. Reed, R. G. Lipes, and C. Wu, "On the application of a fast polinominal transform and the Chinese remainder theorem to compute a two-dimensional convolution," IEEE Trans Acoust., Speech, Signal Processing, vol. ASSP-29, no. 1, pp. 91-97, Feb. 1981.

[79] V. C. Tyree, "Custom large scale integrated circuits for spaceborne SAR processors," in Proc. Synthetic A perture Radar Technology Conf., paper V4 (New Mexico State Univ., Las Cruces, NM, Mar. 1978).

[80] W. J. Van de Lindt, "Digital technique for generating synthetic aperture radar image," IBM J. Res. Develop., pp. 415-432, Sept. 1977.

[81] M. R. Vant, R. W. Herring, and E. Shaw, "Digital processing techniques for satellite borne synthetic aperture radars," Can.J. Remote Sensing, vol. 5, no. 1, 1979.

[82] C. Wu, "A digital system to produce imagery from SAR," in Proc. AIAA Systems Design Driven by Sensors, paper no. 76968 , Oct. 1976.

[83] C. Wu, "Electronic SAR processors for space missions," in Proc. SAR Technology Conf. (New Mexico State Univer., Las Cruces, NM, 1978).

[84] C. Wu, J. Curlander, and A. DiCenzo, "Determination of spacecraft attitude using synthetic aperture radar data," in Proc. Conf. AIAA Sensor System for the 80 's, AIAA paper 80-1922, pp. $57-60,1980$.

[85] C. Wu, K. Y. Liu, and M. Jin, "Modeling and a digital processing algorithm for spaceborne SAR signals," IEEE Trans. Aerosp. Electron. Syst., to be published, Sept. or Nov. 1982.

[86] C. Wu, B. Barkan, W. Karplus, and D. Caswell, "Seasat SAR data reduction using parallel array processors," IEEE Trans. Geosci Remote Sensing, vol. GE-20, no. 3, pp. 352-358, July 1982. 


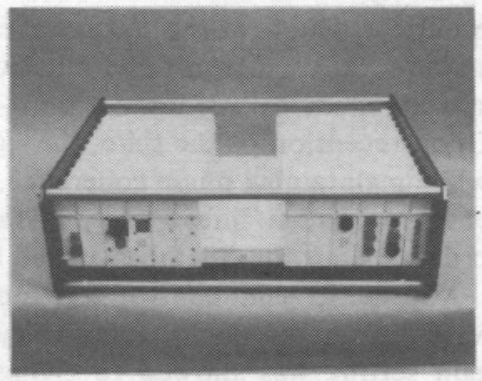

ELECTRONICS

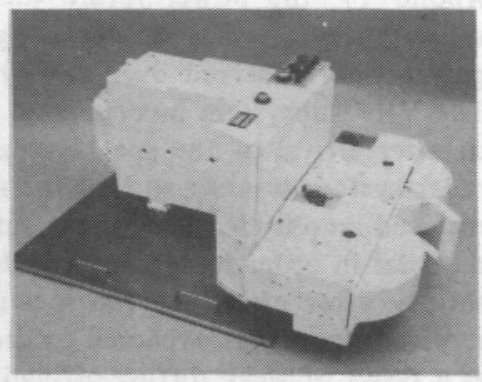

OPTICAL RECORDER

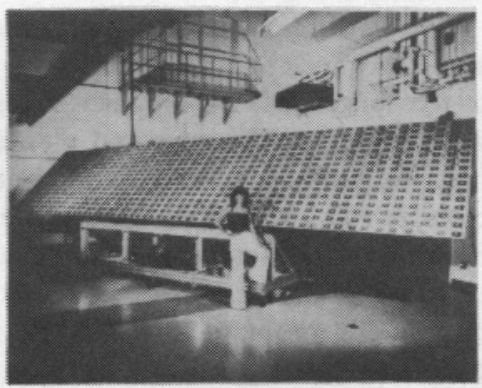

ANTENNA
Weight: $128 \mathrm{~kg}$

Size: $120 \times 85 \times 26 \mathrm{~cm}$

Peak Power Transmitted: 1100 watts

Average Power: 650 watts

Frequency: $1278 \mathrm{MHz}$

Bandwidth: $6 \mathrm{MHz}$

Pulse Length: $30.4 \mu \mathrm{sec}$ (chirped)

PRF: variable between $1464 \mathrm{~Hz}$ and $1824 \mathrm{~Hz}$
Weight: $70 \mathrm{~kg}$

Size (envelope): $108 \times 65 \times 43 \mathrm{~cm}$

Average Power: 135 watts

Film Capacity: 1100 meters

Film Speed: $36 \mathrm{~mm} / \mathrm{sec}$

Operation Time: 8 hours
Weight: $200 \mathrm{~kg}$

Radiating Area Dimensions: $9.44 \times 2.09$ meters Polarization: $\mathrm{HH}$

3ain: $33 \mathrm{~dB}$-dB Beamwidth: $6.2^{\circ} \times 1.2^{\circ}$

Fig. 15. Photographs of the SIR-A sensor and antenna. 

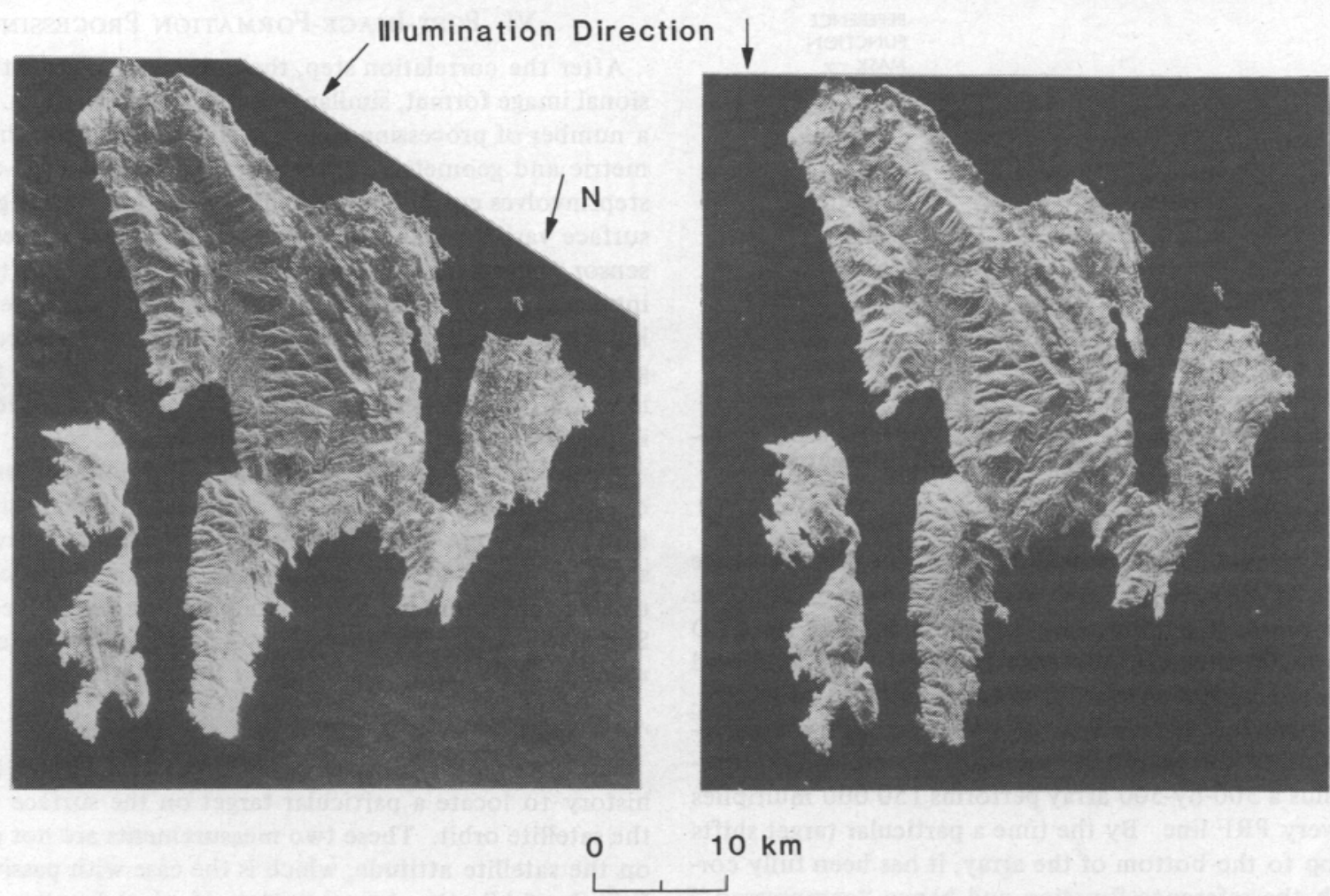

Fig. 29. Stereo pair images of the island of Cephalonia in Greece, acquired with the SIR-A. The angle of convergence is only $5^{\circ}$. This still allows stereo observation. 


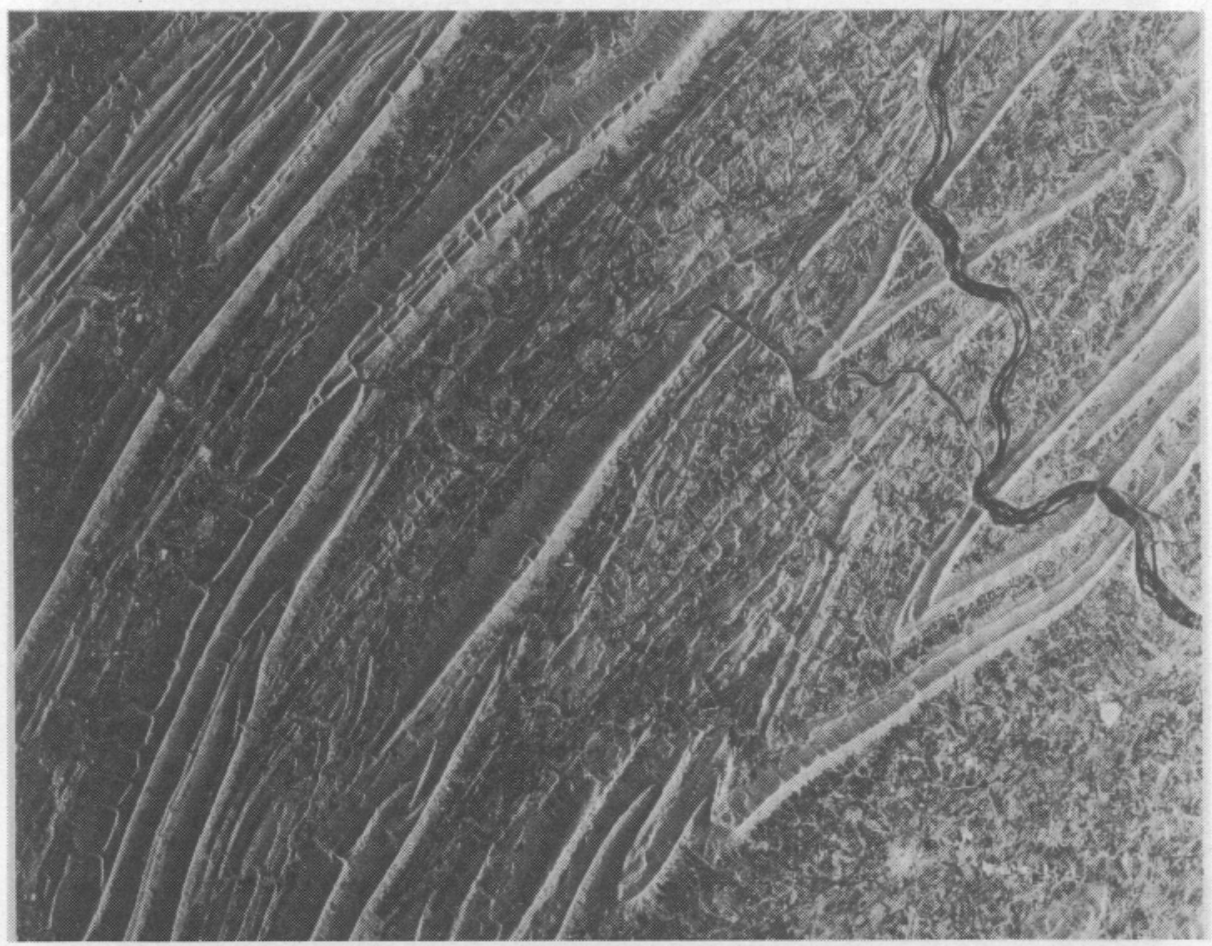

(a)

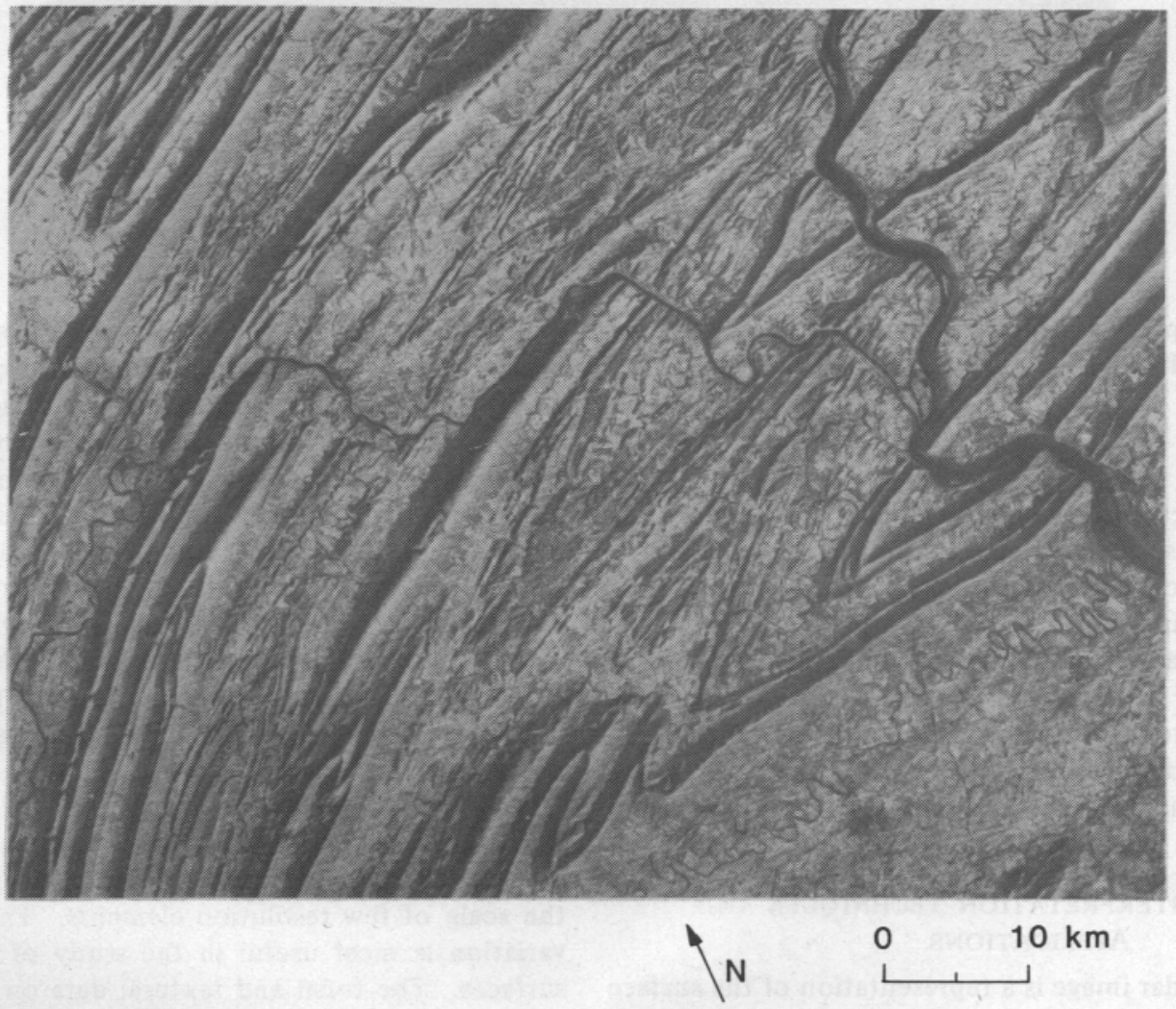

(b)

Fig. 30. Seasat (a) and Landsat (b) images of the Appalachians near Harrisburg, PA. The Susquehanna River is visible in the upper right corner. The pattern of "noses" formed by the mountains and valleys represents plunging structures formed by the folded layers. 


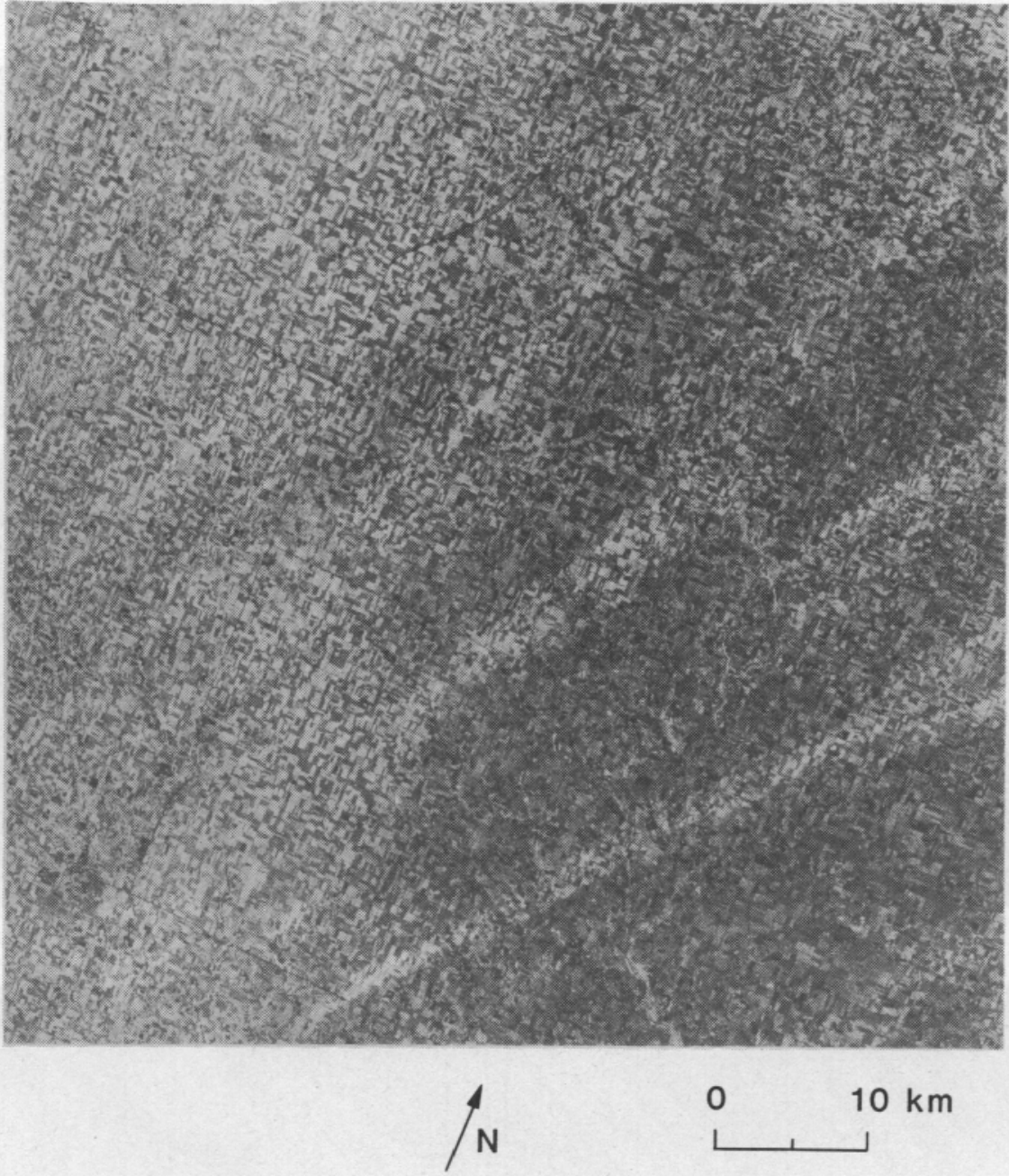

Fig. 31. Seasat image of cultivation fields around Ames, IA. 

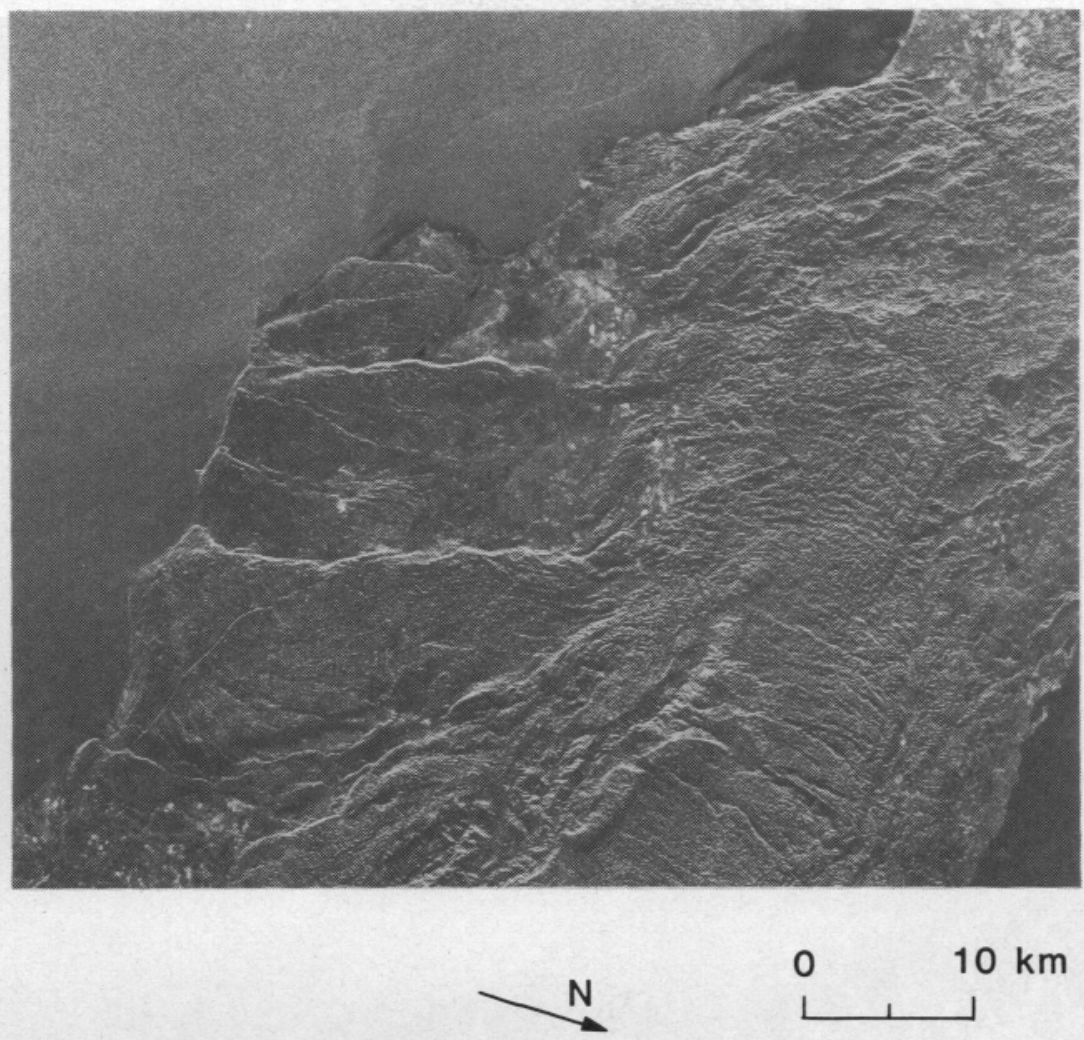

Fig. 32. Seasat image of Central Jamaica. The Blue Mountains in eastern Jamaica represent a pre-Eocene igneous and metamorphic complex. They show as coarse texture. Younger limestones cover most of the rest of the island and form karst topography which shows as fine denture. 

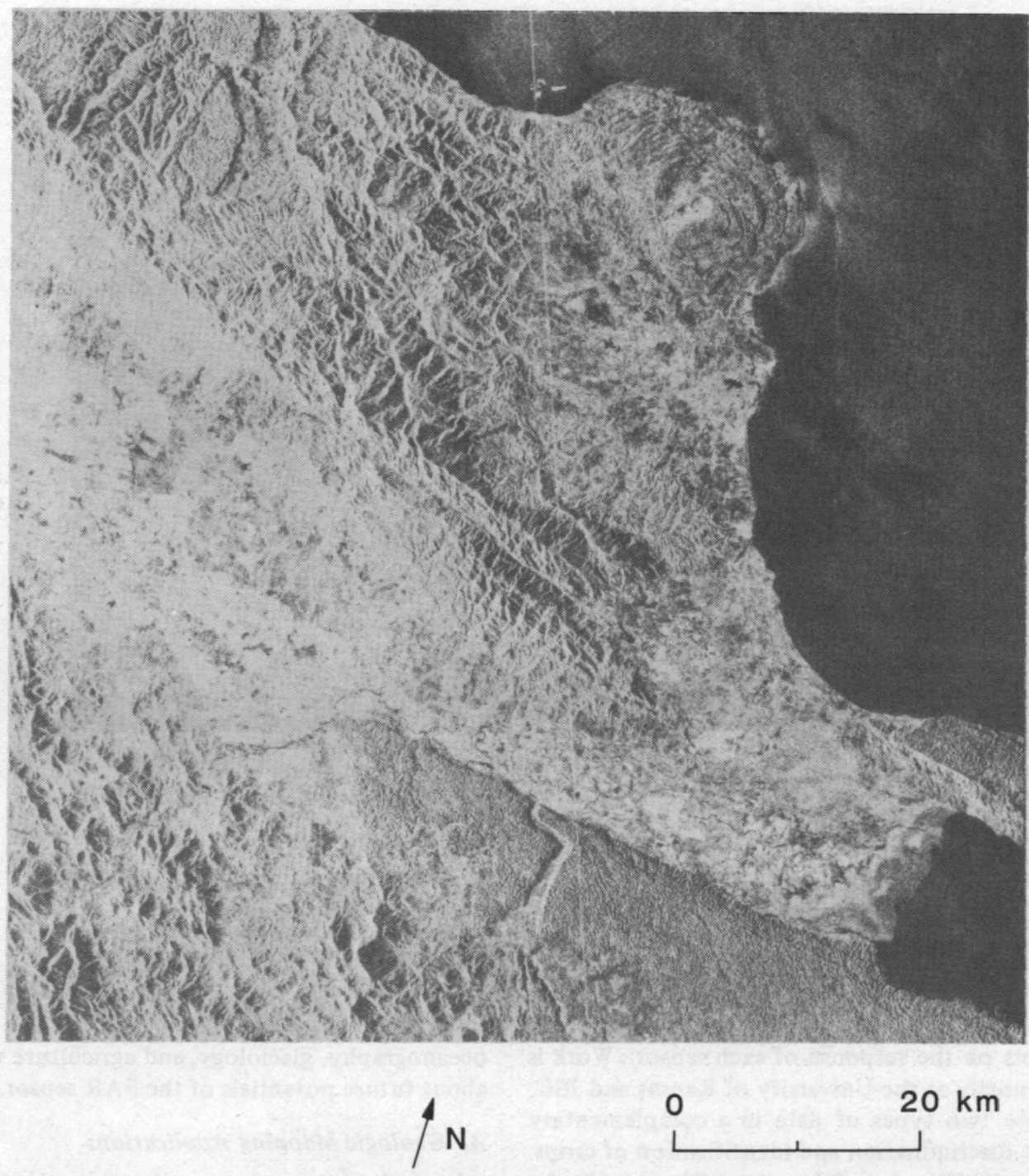

Fig. 33. Seasat image of the northeast part of the Dominican republic. Three major topographic units are visible. The youngest deposits of flat-lying soils and grounds occupy the central region. Tertiary limestones that give rise to karst topography (fine texture) cover the lower right region in the image. Cretaceous and older volcanic rocks underlie the more rugged mountainous basement in the rest of the image. 


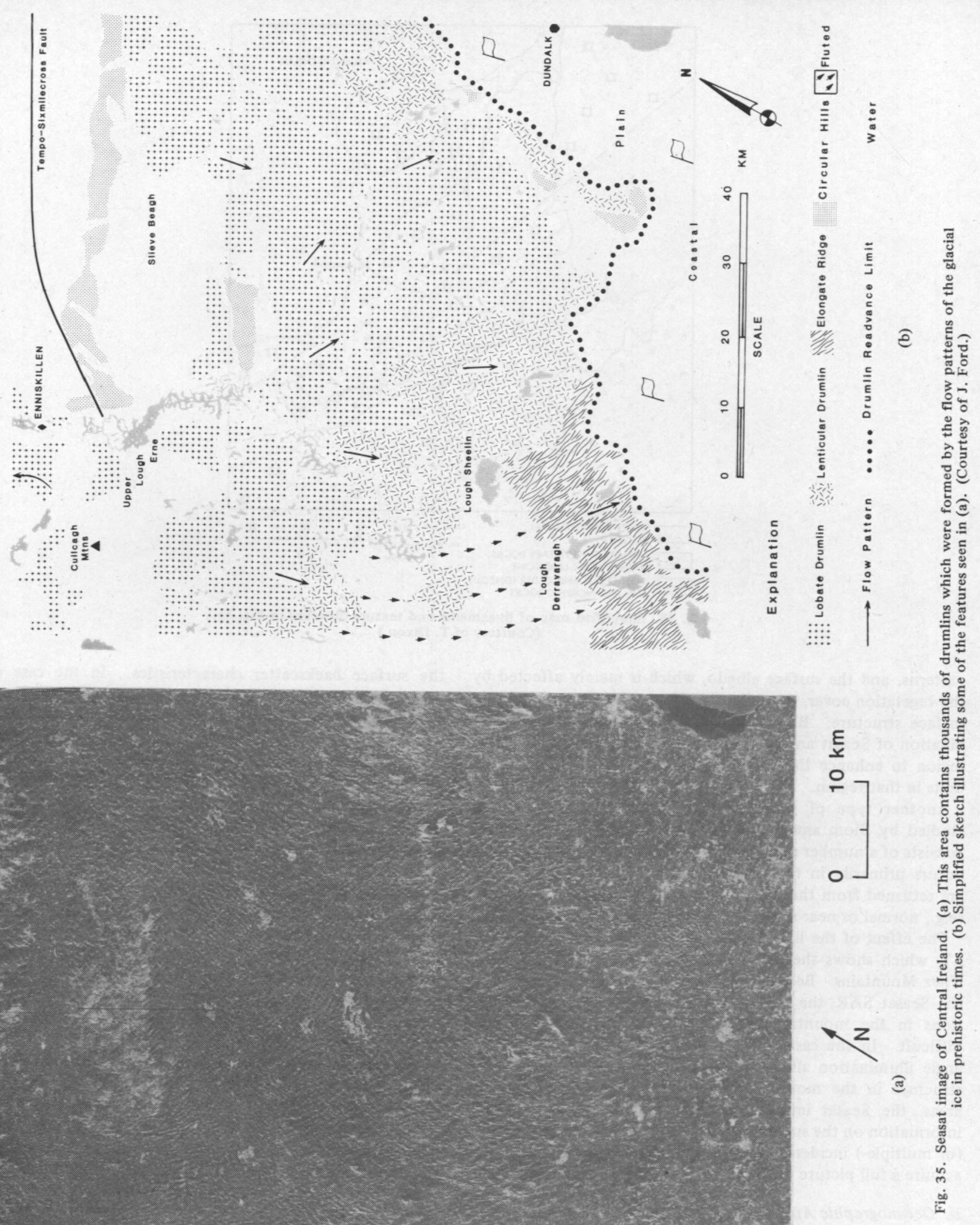



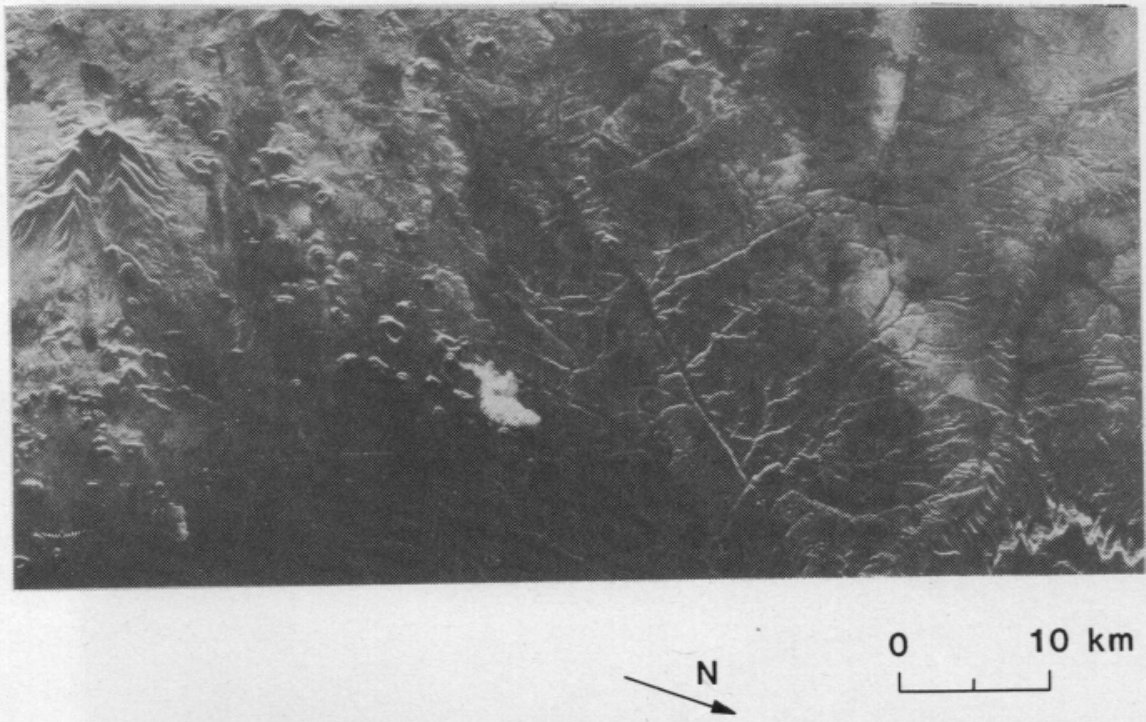

Fig. 36. Seasat image of the San Francisco volcanic field, north of Flagstaff, AZ. Numerous cinder cones and craters are visible. The brightest area corresponds to the lava flow emanating from the SP cinder cone.

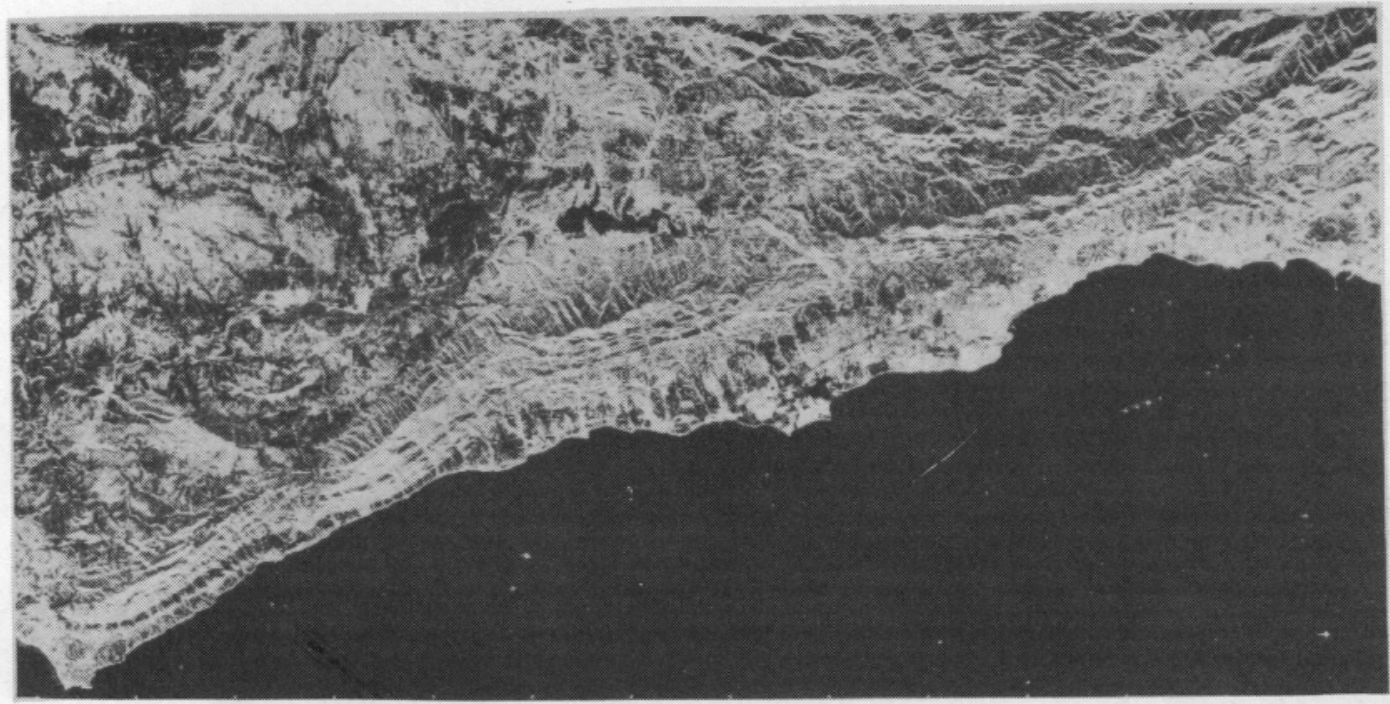

(a)

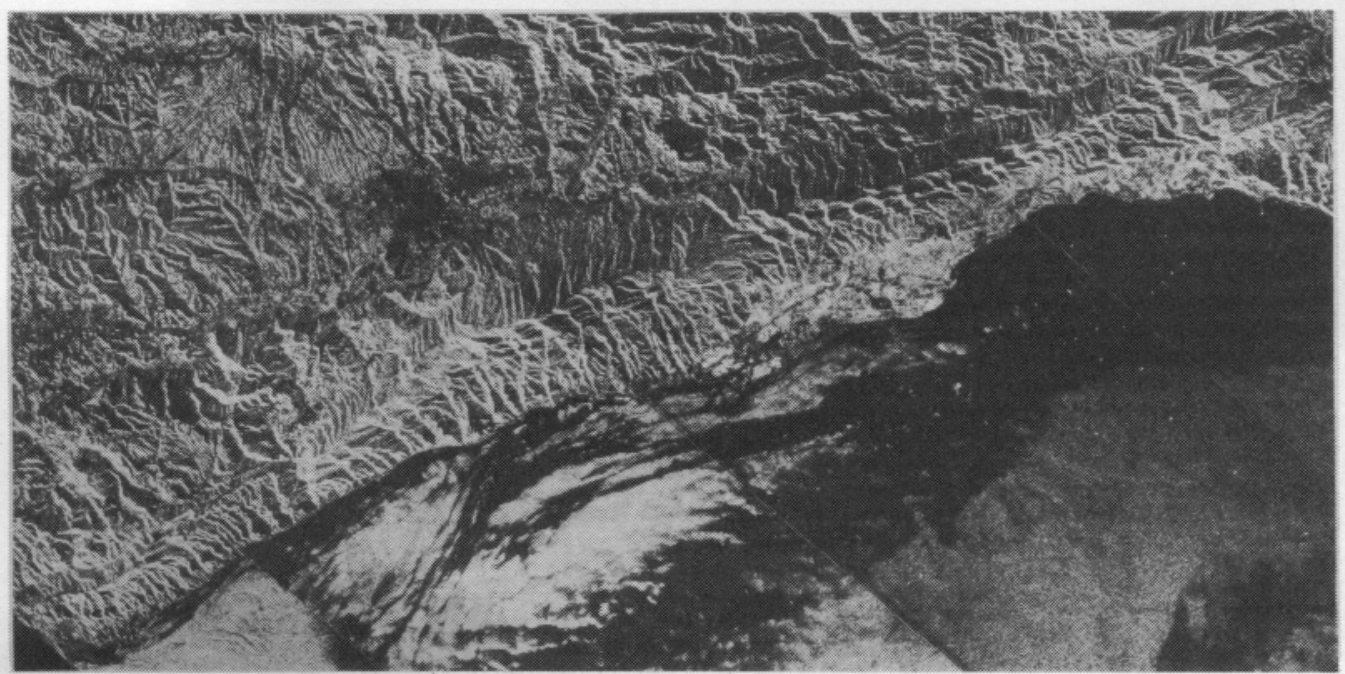

(b)

Fig. 37. Seasat (a) and SIR-A (b) images of the Santa Ynez Mountains along the California coastline near Santa Barbara. The SIR-A image is clearly superior to study the structure in the mountainous region. The Seasat image provides additional information in relatively flat regions.
SIR-A

。 10 km

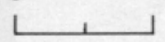

SEASAT 

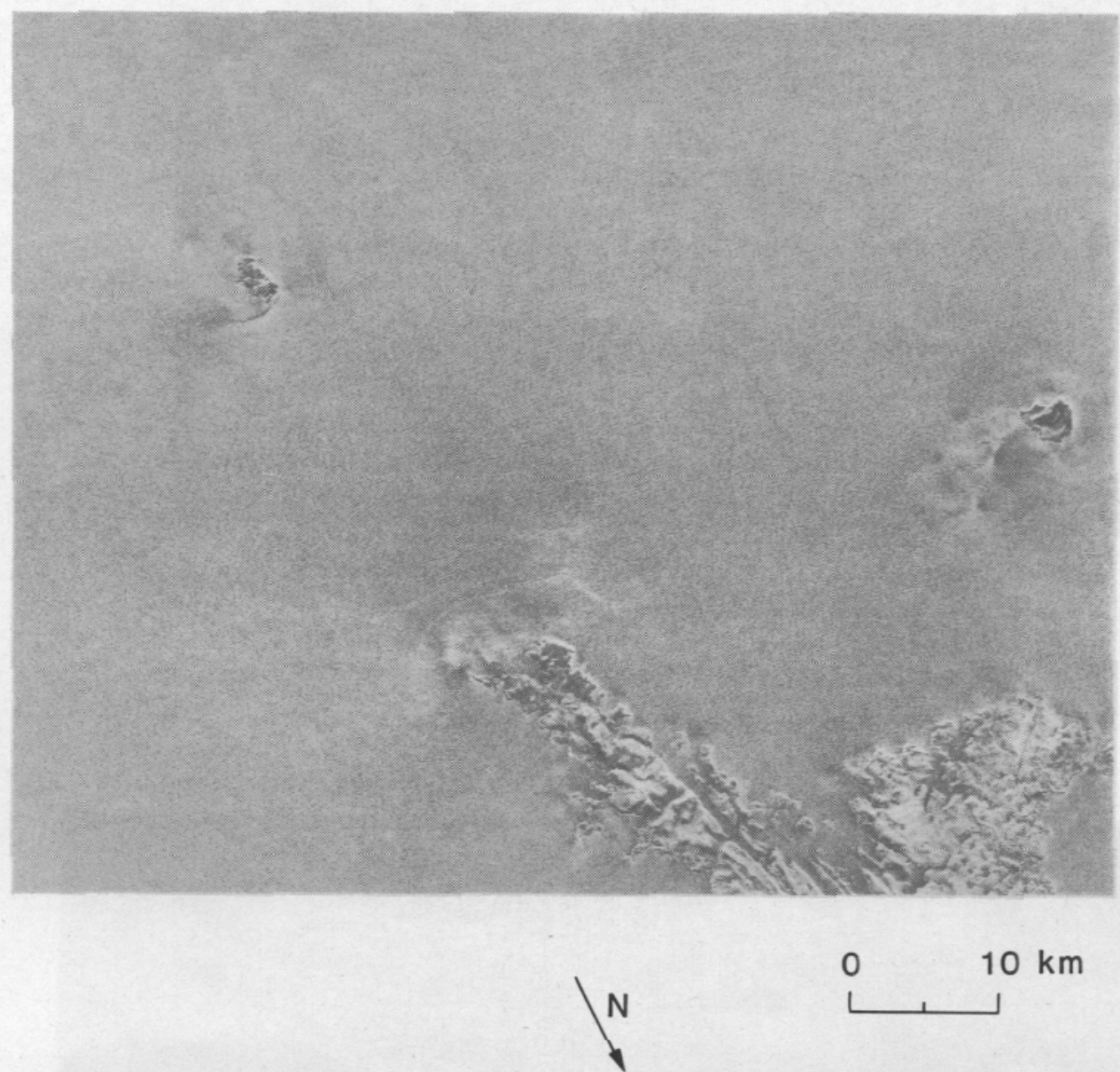

Fig. 38. Seasat image of surface ocean waves near Shetland Island, England. The swell had a wavelength of $300 \mathrm{~m}$. The two small islands are Foule and Fair Isle. Observe the refraction and diffraction of the swell near the coast.
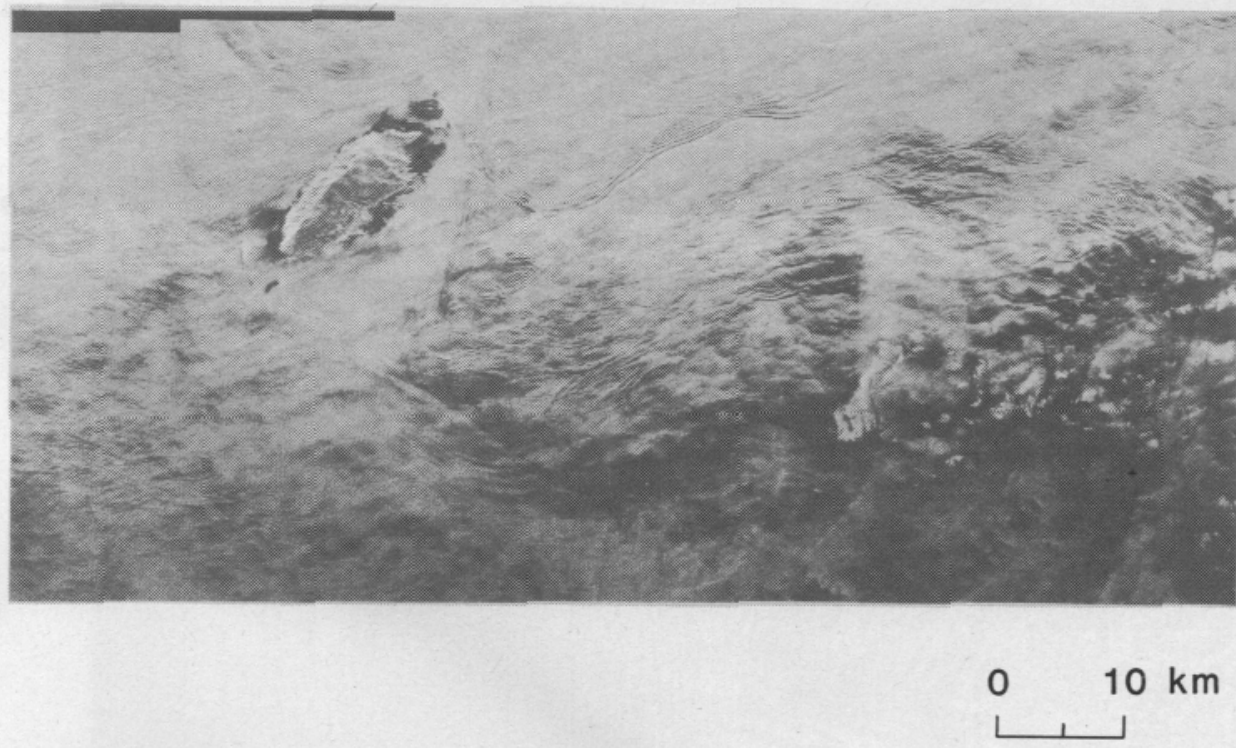

Fig. 39. Seasat image of internal waves near the island of San Nicolas off the coast of California. 

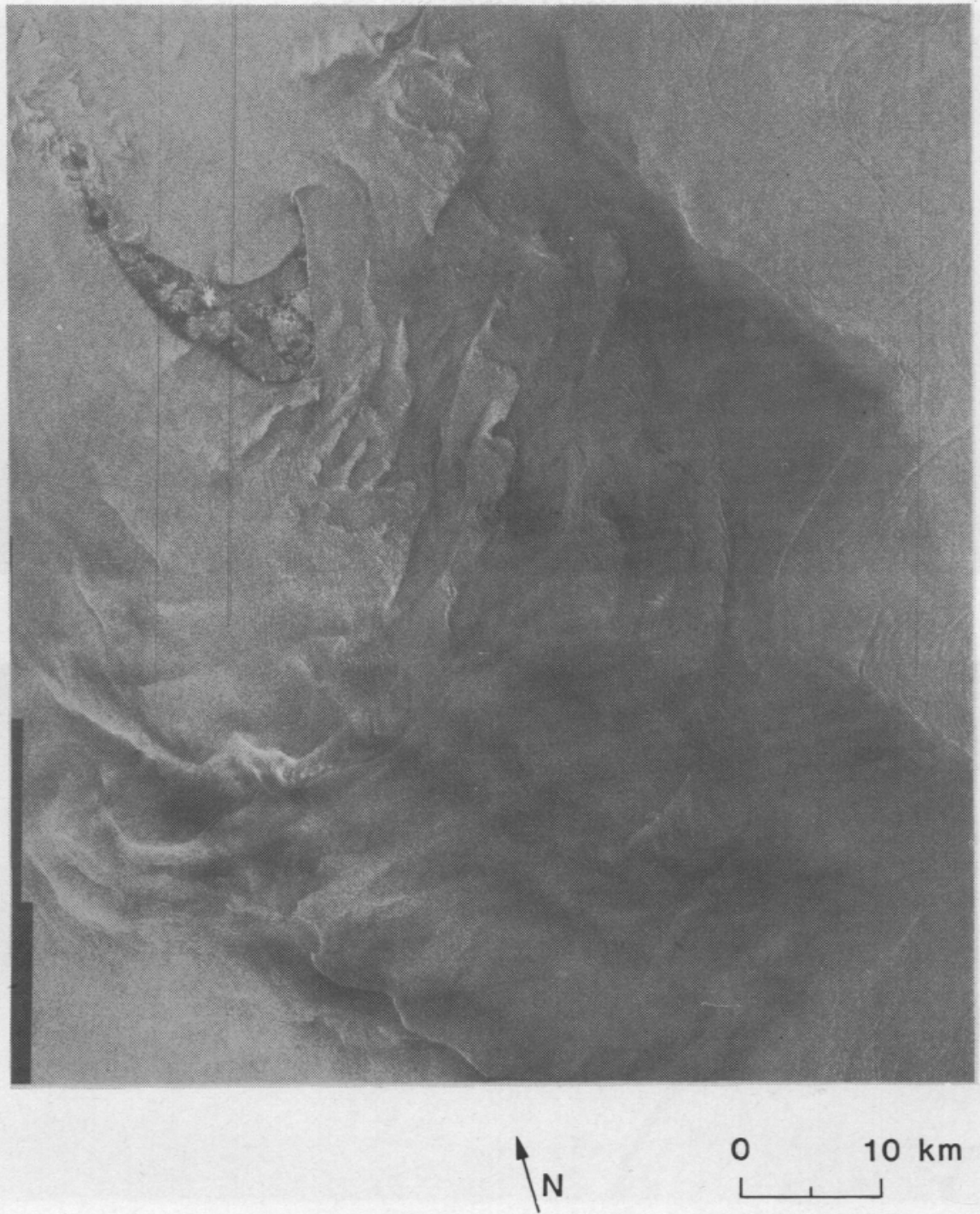

Fig. 40. Seasat image of the Nantucket Island region. The island is visible in the upper left corner of the image. Most of the other patterns observed on the ocean surface reflect the bottom topography in this shallow region. 


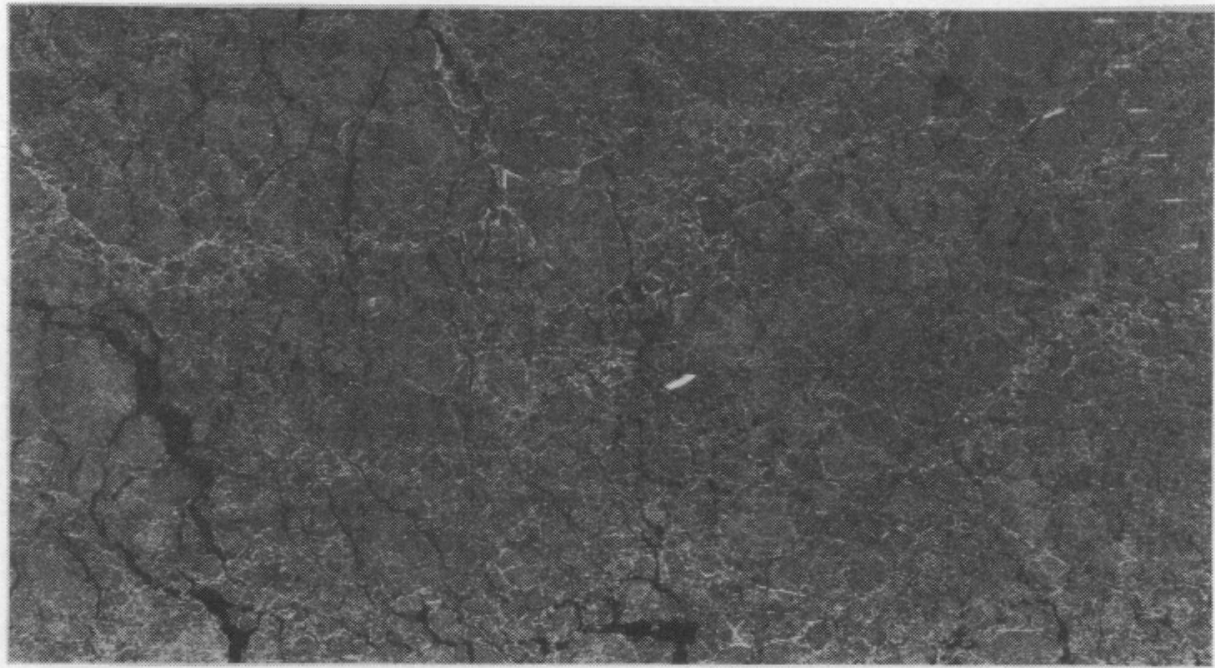

\section{October 1,1978}

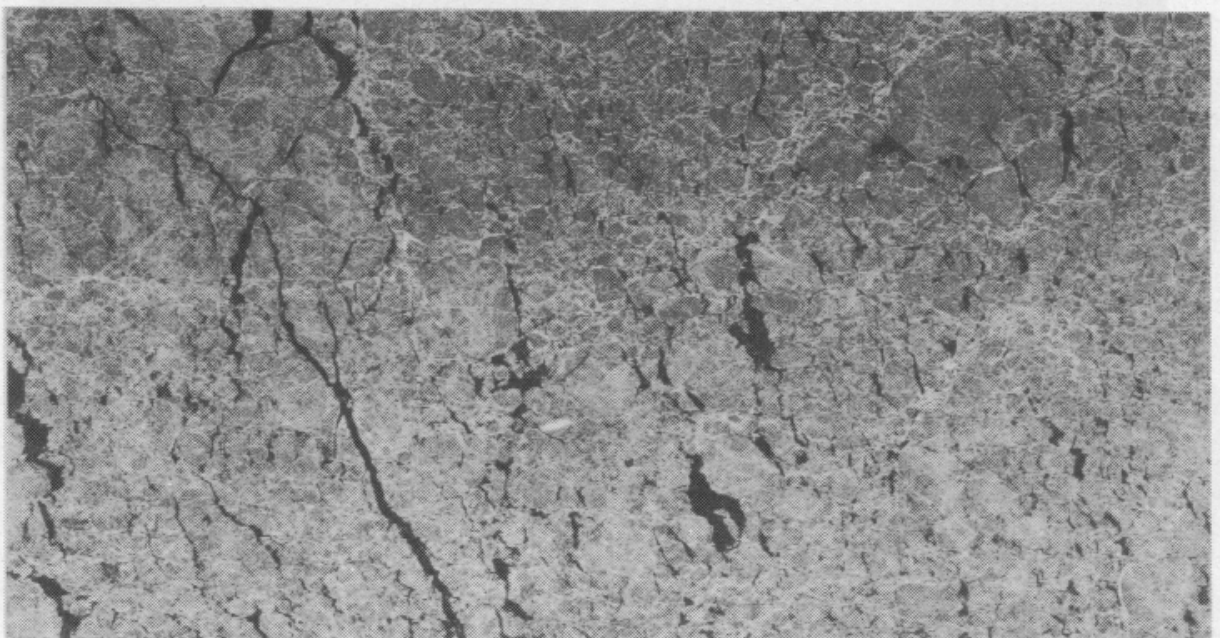

October 7,1978
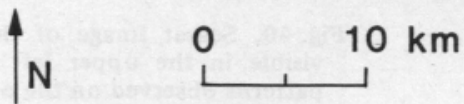

Fig. 41. Seasat image of polar ice floes in the Beaufort Sea acquired six days apart. The bright section is most likely a stranded iceberg. 

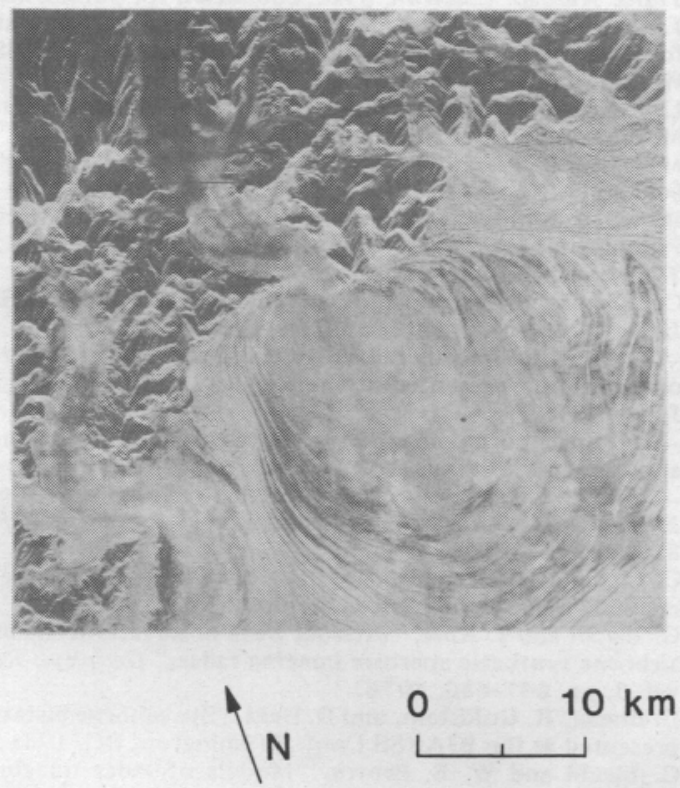

Fig. 43. Seasat image of the Malispina glacier, AK. The ice-floe pattern is visible as a series of bright and dark curvilinear regions. These correspond to the areas of different roughness. 